\title{
Estimação de ordem em modelos AR, ARCH e BEKK-GARCH usando o critério EDC
}

\author{
por
}

Paulo Angelo Alves Resende

Brasília - DF

2014 


\section{Estimação de ordem em modelos AR, ARCH e BEKK-GARCH usando o critério EDC}

Tese apresentada ao Programa de PósGraduação em Matemática da Universidade de Brasília (UnB), como requisito parcial para obtenção do grau de DOUTOR EM MATEMÁTICA.

por

Paulo Angelo Alves Resende

Orientadora:

Chang Chung Yu Dorea

UNIVERSIDADE DE BRASÍLIA

INSTITUTO DE CIÊNCIAS EXATAS

Departamento de MATEMÁtica

Brasília - DF

2014 
À minha mãe, Angela Maria. 


\section{Resumo}

O critério de informação EDC - Efficient Determination Criterion - foi proposto originalmente para definir uma classe de estimadores de ordem para cadeias de Markov de espaço de estados finitos. Nesse trabalho, o conceito de modelos parcialmente aninhados é definido e a classe de estimadores EDC é estendida nesse contexto. Esses resultados são aplicados para estabelecer a consistência forte de um novo estimador de ordem para modelos Autoregressivos (AR) e para demonstrar a consistência forte de uma classe de estimadores de ordem para processos Autoregressivos de Heteroscedasticidade Condicional (ARCH) e para o caso multivariado de modelos Autoregressivos de Heteroscedasticidade Condicional Generalizado na Representação BEKK (BEKK-GARCH). Como resultado imediato, a consistência forte dos estimadores de ordem BIC para ARCH e BEKK-GARCH é estabelecida. Também é ilustrado por meio de simulações numéricas que o estimador de ordem EDC proposto para processos AR apresenta melhor performance que suas principais alternativas, os estimadores baseados nos critérios AIC, BIC e HQC. 


\section{Abstract}

The Efficient Determination Criterion (EDC) was originally stated to define a class of estimators for the order of a Markov chain with finite state space. In this work, we define the concept of partially nested models and extend the class of EDC estimators within this context. This framework is applied to establish the consistency for a new order estimator for Autoregressive process (AR) and to prove the consistency for a class of order estimators for Autoregressive Conditional Heteroskedasticity models (ARCH) and for a multivariate version, the Generalized Autoregressive Conditional Heteroskedasticity in the BEKK representation (BEKK-GARCH). As an immediate consequence, the strong consistency for the $\mathrm{BIC}$ order estimators for ARCH and BEKK-GARCH is established. Also, using numerical simulation, we show that the proposed EDC order estimator for AR performs better than the wide-known alternatives based on the criteria AIC, BIC and HQC. 


\section{Sumário}

$\begin{array}{ll}\text { Introdução } & \text { p. } 7\end{array}$

1 Estimação de ordem em modelos aninhados r 12

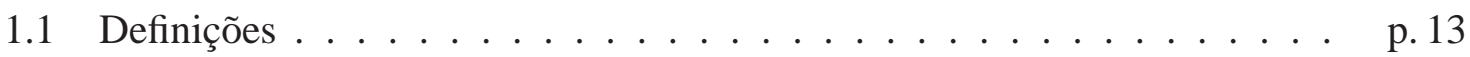

1.2 Consistência de estimadores baseados no critério EDC . . . . . . . p. 17

1.3 Generalização: ordem em modelos parcialmente aninhados . . . . . . . . p. 27

2 Cadeias de Markov de espaço de estados gerais $\quad$ p. 32

2.1 Definições e principais resultados $\ldots \ldots \ldots$ p. . . . . . . . . . . .

2.2 Modelo Autoregressivo $(\mathrm{AR}) \ldots \ldots \ldots \ldots$ p. . . . . . . . . . . . .

2.2 .1 Definições . . . . . . . . . . . . . . . p. 38

2.2.2 Consistência do estimador de ordem de dependência $\ldots . . . \quad$ p. 41

2.2.3 Simulações numéricas . . . . . . . . . . . . . p. 50

2.3 Modelo Autoregressivo de Heteroscedasticidade Condicional (ARCH) . . p.54

2.3.1 Definições . . . . . . . . . . . . . . . p. 54

2.3.2 Consistência do estimador de ordem de dependência $\ldots . . . \quad$ p. . . 58

2.4 Modelo ARCH multivariado generalizado (BEKK-GARCH) . . . . . . p. 70

2.4 .1 Definições . . . . . . . . . . . . . . p. 71

2.4.2 Consistência do estimador de ordem . . . . . . . . p. 75 
Referências Bibliográficas

Apêndice A - Teorema Médio de Cesàro 


\section{Introdução}

No contexto de seleção de modelos, a classe de estimadores de ordem de dependência EDC - Efficient Determination Criterion - foi proposto por Zhao, Dorea \& Gonçalves (2001) como generalização dos critérios BIC e AIC para a estimação de ordem de cadeias de Markov de espaço de estados finitos. Posteriormente, Lopes (2005) estendeu os resultados de Zhao et al. (2001) para cadeias de Markov de espaço de estados enumerável e Dorea (2008) determinou o termo de penalidade ótimo que define o estimador assintoticamente com melhor performance dentro da classe dos estimadores EDC fortemente consistentes.

Com o uso extensivo de simulações numéricas, Resende (2009) verificou a melhor performance do estimador EDC ótimo comparado com as alternativas AIC e BIC. Os resultados empíricos motivaram o presente trabalho, que tem como objetivo generalizar o EDC para a estimação de ordem em modelos markovianos com espaço de estados gerais, que incluem as famílias AR e ARCH, e para a estimação de outros parâmetros, como por exemplo o tamanho do espaço de estados oculto em cadeias de Markov Ocultas.

Uma sequência de variáveis aleatórias $\mathbb{X}=\left\{X_{t}\right\}_{t \in \mathbb{N}}$ é uma cadeia de Markov com espaço de estados finito $E$ e ordem $r$ se, para todo $\left(a_{1}, \ldots, a_{t+1}\right) \in E^{t+1}$,

$$
P\left(X_{t+1}=a_{t+1} \mid X_{1}=a_{1}, \cdots, X_{t}=a_{t}\right)=P\left(X_{t+1}=a_{t+1} \mid X_{t-r+1}=a_{t-r+1}, \cdots, X_{t}=a_{t}\right)
$$

e $r$ é o menor valor com essa propriedade.

Em contextos práticos de modelagem é necessário primeiramente identificar a ordem $r$ da cadeia para posteriormente estimar os parâmetros, que neste caso são as probabilidades de transição.

Esse problema de estimação de ordem foi inicialmente abordado com testes de hipóteses sobre a razão de verossimilhança de Neyman-Pearson por Bartlett (1951), Hoel (1954), Good (1955), Anderson \& Goodman (1957) e Billingsley (1961). Neste caso, observou-se que, 
supondo que $k$ é maior ou igual a ordem verdadeira $r$,

$$
\log \left(\frac{L_{n}(k+1)}{L_{n}(k)}\right) \sim \chi^{2}(\gamma(k+1)-\gamma(k))
$$

para $L_{n}(k)$ a função de verossimilhança estimada de uma amostra $x_{1}, \cdots, x_{n}$, supondo a ordem da cadeia $k$ e $\gamma(k)$ é o número de parâmetros livres. Com isso basta calcular os intervalos de confiança para se obter uma estimativa de $r$.

No contexto de seleção de modelos aninhados, Akaike (1974) propõe o uso do critério de informação AIC, com o simples argumento de tornar a seleção do modelo mais objetiva e não depender de avaliação estatística. Esse critério foi utilizado como base por Tong (1975) para a definição do seguinte estimador de ordem em cadeias de Markov.

$$
\hat{r}_{\text {aic }}=\underset{k \leq K}{\operatorname{argmin}}\{\operatorname{AIC}(k)\}
$$

onde $K$ é uma cota superior conhecida de $r$,

$$
\operatorname{AIC}(k)=-2 \log L_{n}(k)+2 \gamma(k)
$$

e $\gamma(k)=|E|^{k}(|E|-1)$ (isto é, a cardinalidade do conjunto $E$ ). De forma semelhante, Schwarz (1978) propõe o critério de informação BIC e Katz (1981) demonstra a inconsistência do estimador $\hat{r}_{a i c}$ e a consistência fraca do seguinte estimador baseado no BIC.

$$
\hat{r}_{\text {bic }}=\underset{k \leq K}{\operatorname{argmin}}\{\mathrm{BIC}(k)\}
$$

para

$$
\operatorname{BIC}(k)=-2 \log L_{n}(k)+\gamma(k) \log n .
$$

A consistência forte do $\hat{r}_{b i c}$ foi demonstrada por Finesso (1990) e posteriormente, sem a hipótese de existência de um limitante superior $K$, por Csiszár \& Shields (2000).

Em seus trabalhos, Katz (1981) e Csiszár \& Shields (2000), apontam que o estimador BIC possui uma tendência a subestimar a ordem verdadeira de cadeias de Markov em amostras pequenas, o que sugere a necessidade de demonstrar a consistência forte para termos de penalidades menores que $\gamma(k) \log n$. Nesse sentido, Zhao et al. (2001) generalizam os 
critérios AIC e BIC, criando a classe de estimadores

$$
\hat{r}_{e d c}=\underset{k \leq K}{\operatorname{argmin}}\{\operatorname{EDC}(k)\}
$$

para

$$
\operatorname{EDC}(k)=-2 \log L_{n}(k)+\gamma(k) c_{n}
$$

e demonstram a consistência forte do estimador $\hat{r}_{e d c}$ para $c_{n}$ satisfazendo

$$
\lim _{n \rightarrow \infty} \frac{c_{n}}{n}=0 \quad e \quad \liminf _{n \rightarrow \infty} \frac{c_{n}}{\log \log n}=\infty
$$

Posteriormente, Dorea (2008) demonstra a consistência forte para $\hat{r}_{e d c}$ quando $c_{n}$ satisfazendo

$$
\lim _{n \rightarrow \infty} \frac{c_{n}}{n}=0 \quad e \quad \liminf _{n \rightarrow \infty} \frac{c_{n}}{\log \log n} \geq 2 \frac{|E|}{|E|-1}
$$

e propõe o estimador ótimo $\hat{r}_{\text {opt }}$ com o termo de penalidade $\gamma(k) c_{n}=2|E|^{k+1} \log \log n$. Usando simulações numéricas, Resende (2009) comprovou a melhor performance do estimador $\hat{r}_{o p t}$ e verificou que essa eficiência relativa é substancialmente superior à medida que se considera modelos com maior complexidade (número de parâmetros livres).

Observando que os estimadores citados são baseados no comportamento da verossimilhança, que é o mesmo fundamento utilizado nos testes de hipóteses, Baigorri, Gonçalves \& Resende (2014) propõem o estimador GDL, baseado na comparação das distribuições empíricas condicionadas usando a divergência chi-quadrado. Em simulações numéricas, o GDL apresentou performance superior às demais alternativas. Entretanto, o método utiliza diretamente em sua definição a finitude do espaço de estados da cadeia de Markov, o que torna improvável uma generalização a outros processos.

Embora os trabalhos citados usem o conceito de modelos aninhados, os resultados são sempre particularizados para o processo considerado. Dessa forma, nessa tese, os resultados existentes foram aperfeiçoados para tratar a consistência do estimador $\hat{r}_{e d c}$ baseada, basicamente, em hipóteses sobre regularidade e comportamento assintótico de funções $\log L_{n, k}$, que possuem propriedades semelhantes à log-verossimilhança.

O ferramental desenvolvido é aplicado para obter a consistência de estimadores de ordem para os processos Autoregressivos (AR), Autoregressivos de Heteroscedasticidade Condicio- 
nal (ARCH) e ARCH multivariado generalizado na representação BEKK (BEKK-GARCH).

Em simulações numéricas, o estimador obtido para processos Autoregressivos apresentou, no geral, melhor performance quando comparado com os estimadores AIC (Akaike 1974), BIC (Shibata 1976) e HQC (Hannan \& Quinn 1979).

Ressalta-se que, para processos ARCH e BEKK-GARCH, até então não é conhecida na literatura a existência de estimadores fortemente consistentes. Assim, esse resultado é inédito e de alta relevância.

Para os processos AR e ARCH, a ordem a ser estimada é um parâmetro univariado, o que permite a definição de sequência de modelos aninhados, onde o EDC é originalmente definido por Zhao et al. (2001). Entretanto, em modelos BEKK-GARCH, a ordem a ser estimada é um parâmetro com duas variáveis (de forma semelhante ocorre com modelos ARMA). Nesse sentido, o conceito de sequência de modelos aninhados é estendido a classe de modelos parcialmente aninhados, permitindo assim a definição do EDC nesse novo contexto para a estimação de ordens multivariadas que tem como caso particular modelos BEKK-GARCH.

A técnica utilizada nos três casos para se obter o comportamento assintótico de $\log L_{n, k}$ é essencialmente a mesma, que basicamente consiste na utilização do comportamento assintótico das derivadas de primeira ordem de $\log L_{n, k}$ para concluir determinado comportamento assintótico dos estimadores dos parâmetros da densidade em questão e, a partir desse comportamento, conclui-se o comportamento assintótico de $\log L_{n, k}$.

Essa técnica foi empregada por Nishii (1988) para a estimação de dimensão em modelos aninhados no caso particular de sequências de variáveis independentes e identicamente distribuídas (i.i.d.) e considerando $\log L_{n, k}$ a $\log$-verossimilhança. Posteriormente, Finesso (1990) baseou-se nessa técnica para demonstrar a consistência forte do estimador de ordem BIC para cadeias de Markov. Vale ressaltar que Basawa \& Heyde (1976) utilizam de técnica semelhante para concluir a normalidade assintótica do estimador do parâmetro da densidade a partir do comportamento assintótico das derivadas de primeira ordem da logverossimilhança.

Geralmente as derivadas de $\log L_{n, k}$ são mais simples, isso motiva o uso desse caminho. Outro fato importante é que várias hipóteses para a aplicação do método são usadas para a obtenção da normalidade assintótica dos estimadores e por isso frequentemente suas 
demonstrações são encontradas na literatura.

No Capítulo 1 os conceitos de modelos aninhados e ordem são generalizados e formalizados, e alguns resultados gerais relativos à consistência do estimador EDC são apresentados. No Capítulo 2 são introduzidos conceitos e resultados de cadeias de Markov com espaço de estados gerais. Esses resultados são empregados para obter o comportamento assintótico das respectivas funções $\log L_{n, k}$ definidas para os processos AR, ARCH e BEKK-GARCH, para então concluir os casos de consistência forte do EDC. Nos apêndices são encontrados resultados auxiliares, que são utilizados nas demonstrações. 


\section{$1 \quad$ Estimação de ordem em modelos aninhados}

Os estudos pioneiros em estimação de ordem para diversos processos estocásticos clássicos foram focados na definição de condições para a aplicação de testes de hipóteses no apoio ao trabalho de análise e decisão (Bartlett 1951, Whittle 1951). Em um segundo momento, ainda com uma visão estatística para abordar o problema, tiveram os trabalhos no desenvolvimento de critérios de informação no contexto de seleção de modelos aninhados (Akaike 1974, Schwarz 1978). Com a necessidade de se obter um melhor entendimento, alguns autores utilizaram com sucesso uma abordagem analítica na avaliação do comportamento assintótico dos objetos envolvidos para concluir aspectos de consistência dos estimadores (Nishii 1988, Finesso 1990). Nesse cenário, Zhao et al. (2001) propõem o EDC como uma generalização dos critérios de informação que usam o método da log-verossimilhança penalizada.

Ao longo das últimas quatro décadas, os critérios de informação vêm sendo implementados nos mais diversos processos e situações (Tong 1975, Shibata 1976, Ozaki 1977, Ogata 1980, Zhao, Dorea \& Gonçalves 2001, Lopes 2005, Polansky 2007). Entretanto, não houve uma proposta efetiva de ferramental que facilite o estudo da consistência de estimadores de ordem em novos casos. Nesse sentido, na Seção 1.1 os conceitos de modelos aninhados e ordem são apresentados de forma mais geral. A Seção 1.2 contém a definição do estimador de ordem EDC para esse contexto e o aperfeiçoamento e generalização dos resultados de Nishii (1988) e Dorea (2008). Com isso é possível obter os casos de consistência do estimador de ordem EDC baseado, basicamente, em condições sobre as funções $\log L_{n, k}$, que em muitas situações podem ser as funções log-verossimilhança. A última seção contém a generalização de sequência de modelos aninhados para classe de modelos parcialmente 
aninhados e a reapresentação dos resultados da Seção 1.2 nesse cenário ainda mais geral.

\subsection{Definições}

A maioria dos autores citados que aplicam algum critério de informação não necessitam tratar diretamente em caso geral de seleção de modelos, com isso não definem modelos aninhados. Por outro lado, Akaike (1974) e Schwarz (1978) usam livremente a palavra "modelo" sem definição explícita do objeto. Na demonstração da consistência forte do estimador BIC para cadeias de Markov, Csiszár \& Shields (2000) definem conjunto de modelos estatísticos de índice $k$ como a classe de processos que satisfaz a propriedade de Markov de ordem $k$.

Com o objetivo de generalizar os critérios de informação para o caso i.i.d., Nishii (1988) define um conjunto de modelos como uma família de densidades, mas não se depara com o problema de definição de aninhamento para sequências de variáveis aleatórias que possuem dependência. A definição abaixo é a generalização da definição de Nishii. Observa-se que, como as densidades são em relação a uma medida fixada $v$, pode-se valer disso para simplificar a expressão da $\log$-verossimilhança e possivelmente das funções $\log L_{n, k}$ na aplicação dos resultados subsequentes.

Definição 1.1. Para um processo estocástico $\mathbb{X}=\left\{X_{t}\right\}_{t \in \mathbb{N}}, E \subseteq \mathbb{R}^{p}$ o conjunto de possíveis valores de $X_{t}, v$ uma medida fixada em E, assumimos uma família de modelos estatísticos para $\mathbb{X}$ como

$$
M=\left(f\left(x_{1}^{n}, \theta, n\right), \Theta\right), \theta \in \Theta, \forall n \geq 1
$$

onde $f\left(x_{1}^{n}, \theta, n\right)$ representa o conjunto de possíveis densidades de $x_{1}^{n}$ com respeito à medida produto em $E^{n}$, que depende do parâmetro $\theta \in \Theta \subseteq \mathbb{R}^{d}$ e $x_{1}^{n}=x_{1} x_{2} \ldots x_{n}$ é uma possível realização de $\mathbb{X}$. Para simplificar a notação, denotamos $f\left(x_{1}^{n}, \theta, n\right)$ por $f\left(x_{1}^{n}, \theta\right)$. Os elementos de $M$ podem ser denotados apenas pelas suas densidades, exemplo $m=f\left(x_{1}^{n}, \theta_{0}\right) \in M$.

O fundamento do conceito de "aninhamento" foi utilizado em hipóteses aninhadas para a aplicação dos testes na razão da log-verossimilhança de Neyman-Pearson, como exemplo em Hoel (1954). A seguir apresentamos uma generalização desse conceito e a definição de ordem para sequência de modelos aninhados. 
Definição 1.2. Para

$$
\begin{gathered}
M_{i}=\left(f_{i}\left(x_{1}^{n}, \theta\right), \Theta_{i}\right), \theta \in \Theta_{i}, n \geq 1, \\
M_{j}=\left(f_{j}\left(x_{1}^{n}, \theta\right), \Theta_{j}\right), \theta \in \Theta_{j}, n \geq 1 .
\end{gathered}
$$

(i) Dizemos que $M_{i} \subseteq M_{j}$ se e somente se $\Theta_{i} \subseteq \Theta_{j}$ e, para todo $\theta \in \Theta_{i}, n \in \mathbb{N}$ e $x_{1}^{n} \in E^{n}$ existe $c_{1} \in(0, \infty)$, que não depende de $n$, tal que, se, para $c_{2}$ suficientemente grande, $n>c_{2} \in \mathbb{N}$,

$$
\frac{f_{i}\left(x_{1}^{n}, \theta\right)}{f_{j}\left(x_{1}^{n}, \theta\right)}=c_{1} .
$$

Neste caso denotamos por $f_{i}(\cdot, \theta) \simeq f_{j}(\cdot, \theta)$.

(ii) $\mathbb{M}=\left\{M_{k}\right\}_{k \in \mathbb{N}}$ é uma sequência de modelos aninhados se $M_{k} \subseteq M_{k+1}$ para todo $k \in \mathbb{N}$.

(iii) Se $\mathbb{M}$ é sequência de modelos aninhados e $m \in \bigcup_{k=0}^{\infty} M_{k}$, dizemos que mé de ordem 0 se $m \in M_{0}$ e é de ordem $r>0$ se $m \in M_{r}$ e $m \notin M_{r-1}$.

(iv) Se $\mathbb{M}=\left\{M_{k}\right\}_{k \in \mathbb{N}}$ é sequência de modelos aninhados, denotamos $\gamma(k)=\operatorname{dim}\left(\Theta_{k}\right)$.

Observa-se que em (1.1), bastaria

$$
\lim _{n \rightarrow \infty} \frac{f_{i}\left(x_{1}^{n}, \theta\right)}{f_{j}\left(x_{1}^{n}, \theta\right)}=c_{1}
$$

Entretanto, a forma como foi definido é suficiente e mais simples. Essencialmente, o objetivo é definir equivalência das densidades conjuntas em função do comportamento assintótico quase certo, em outras palavras $f_{i}(n)=O\left(f_{j}(n)\right)$ para quase toda realização do processo.

Mesmo no contexto de seleção de modelos, o termo "verossimilhança" (ou "verossimilhança estimada") comumente se refere a uma função. Entretanto, implicitamente trata-se de uma classe de funções que depende da hipótese assumida como verdadeira e do tamanho da amostra. Caso contrário, encontramos dificuldades na definição explícita do domínio dessa função. Para ilustrar essa situação, suponha que temos uma cadeia de Markov de ordem $r$ com espaço de estados finito $E$. Considere $\mathbb{M}$ sua respectiva sequência de modelos aninhados, $\theta_{k} \in \Theta_{k}$ uma matriz de transição e $p_{i, j}\left(\theta_{k}\right)$ as respectivas probabilidades de transição considerando $i \in E^{k}$. Para uma amostra $x_{1}^{n}=x_{1} x_{2} \ldots x_{n}$, fixando $n$ e $k$, a verossimilhança é 
dada por

$$
C\left(x_{1}^{k}\right) \prod_{i=k+1}^{n} p_{x_{i-k}^{i-1}, x_{i}}\left(\theta_{k}\right) .
$$

Se por outro lado supormos a ordem $k+1$ temos

$$
C\left(x_{1}^{k+1}\right) \prod_{i=k+2}^{n} p_{x_{i-k-1}^{i-1}, x_{i}}\left(\theta_{k+1}\right) .
$$

Mesmo desprezando os termos $C(\cdot)$, se ambas expressões são referentes à imagem de uma única função, teríamos que permitir amostras de tamanhos arbitrários e inserir $n$ e $k$ no domínio da função. Neste caso, uma alternativa de definição para a função verossimilhança seria

$$
\begin{gathered}
L: E^{\infty} \times \Theta \times \mathbb{N} \times \mathbb{N} \rightarrow \mathbb{R} \\
L\left(x_{1}^{n}, \theta_{k}, n, k\right)=C\left(x_{1}^{k}\right) \prod_{i=k+1}^{n} p_{x_{i-k}^{i-1}, x_{i}}\left(\theta_{k}\right)
\end{gathered}
$$

para $\Theta=\cup_{i=0}^{\infty} \Theta_{k}$ e considerando as devidas imersões de $\Theta_{k} \subset \Theta$ e $E^{n} \subset E^{\infty}$. Entretanto, dentre possíveis outras, teríamos dificuldades em manipular as derivadas de $L$ em relação a $\theta$. Como exemplo, manipular a matriz Hessiana $D_{\theta}^{2} L\left(\hat{\theta}_{k}\right)$ para um estimador de máxima verossimilhança $\hat{\theta}_{k} \in \Theta_{k} \subset \Theta$.

Além disso, em várias implementações de estimadores de ordem encontradas na literatura, é comum "desprezar" alguns fatores da verossimilhança ou até mesmo "aproximar" a verossimilhança por outra função. Esse fato evidencia que a estimação da ordem, embora tenha suas raízes no uso da verossimilhança, não necessita que a função a ser penalizada seja precisamente a log-verossimilhança.

Nesse sentido, definimos abaixo a classe de funções $\left\{L_{n, k}\right\}$, ou em uma nomenclatura mais simples, funções $L_{n, k}$, que possui as propriedades adequadas para a definição do estimador EDC e estabelecimento dos resultados subsequentes. No geral, as funções $L_{n, k} \operatorname{sim}$ plesmente se referem à função verossimilhança para seus respectivos $n$ e $k$.

Definição 1.3. Para $x_{1}^{n}$ uma realização de $\mathbb{X}, \mathbb{M}=\left\{M_{k}\right\}_{k \in \mathbb{N}}$ uma sequência de modelos aninhados e $\hat{\theta}_{k}$ o estimador de máxima verossimilhança considerando $k$ a ordem verdadeira, definimos a família de funções $\left\{L_{n, k}\right\}$, com $L_{n, k}: E^{n} \times \Theta_{k} \rightarrow \mathbb{R}$, que satisfaça

$$
L_{n, k}\left(x_{1}^{n}, \hat{\theta}_{k}\right)=\sup _{\theta \in \Theta_{k}}\left\{L_{n, k}\left(x_{1}^{n}, \theta\right)\right\}
$$


$e$, para todo $k, \theta \in \Theta_{k}$, e $l \geq k$,

$$
L_{n, l}(\theta) \geq L_{n, k}(\theta) \quad e \quad \lim _{n \rightarrow \infty} \frac{L_{n, l}(\theta)}{L_{n, k}(\theta)}<\infty .
$$

Quando não houver dúvidas, utilizamos $L_{n}\left(\hat{\theta}_{k}\right)=L_{n, k}\left(x_{1}^{n}, \hat{\theta}_{k}\right)$ ou $L_{n, k}(\theta)=L_{n, k}\left(x_{1}^{n}, \theta\right)$.

Usando (1.3) temos que, para todo $l \geq k, \theta \in \Theta_{k}$ e sequência $h_{n}$ tal que $\lim _{n \rightarrow \infty} h_{n}=\infty$,

$$
0 \leq \frac{\log L_{n, l}(\theta)-\log L_{n, k}(\theta)}{h_{n}} \rightarrow 0 .
$$

Essa relação é utilizada para comparar as diferenças $\log L_{n}\left(\hat{\theta}_{k}\right)-\log L_{n}\left(\hat{\theta}_{r}\right)$ no que segue.

Exemplo 1.1. Considere a família de modelos “Weibull”, $M_{W}$, com

$$
\begin{array}{r}
E=\mathbb{R}^{+}, \\
f\left(x_{1}^{n}, \theta\right)=\prod_{k=1}^{n} \frac{\theta_{1}}{\theta_{2}} x_{k}^{\theta_{1}-1} e^{-\frac{x_{k}^{\theta_{1}}}{\theta_{2}},} \\
\forall \theta=\left(\theta_{1}, \theta_{2}\right) \in \mathbb{R}^{+} \times \mathbb{R}^{+} .
\end{array}
$$

Com a restrição abaixo, temos a família de modelos "exponencial”, $M_{e}, e M_{e} \subseteq M_{W}$.

$$
\forall \theta=\left(1, \theta_{2}\right), \theta_{2} \in \mathbb{R}^{+}
$$

No contexto apresentado, além dos casos i.i.d. como ilustrado no exemplo acima, é possível inserir os problemas de identificação de ordem de dependência para cadeias de Markov, cadeias de Markov ocultas [ordem de dependência oculta], processos Autoregressivos (AR), processos Autoregressivos de Heteroscedasticidade Condicional (ARCH), além de outros casos particulares pouco difundidos como Raftery (1985), Logan (1981) e Pegram (1980). O ferramental também pode ser utilizado além dos casos de ordem de dependência, como no problema da identificação do tamanho do espaço de estados oculto de cadeias de Markov ocultas. 


\subsection{Consistência de estimadores baseados no critério EDC}

No que segue, definimos o estimador de ordem EDC em sequências de modelos aninhados, que não apresenta diferença significativa em relação à definição de Zhao et al. (2001).

Definição 1.4. Para $\mathbb{M}$ uma sequência de modelos aninhados, $m_{r} \in \bigcup_{k=0}^{\infty} M_{k}$ de ordem $r$ e $K \geq r$, definimos o estimador EDC de $r$ por

$$
\hat{r}_{e d c}=\underset{k \leq K}{\operatorname{argmin}}\{\operatorname{EDC}(k)\}
$$

onde

$$
\operatorname{EDC}(k)=-\log L_{n}\left(\hat{\theta}_{k}\right)+c_{n} \gamma(k),
$$

$\left\{c_{n}\right\}_{n \in \mathbb{N}}$ é sequência de números positivos e $L_{n}\left(\hat{\theta}_{k}\right)$ é como na Definição 1.3.

A rigor, $\gamma(k)$ pode ser qualquer função crescente em $k$. Na literatura é comum adotar $c_{n}$ como sequência que não depende de $x_{1}^{n}$ e/ou $k$. Entretanto essa restrição não é necessária.

O objetivo do Teorema abaixo é concluir os casos de consistência do estimador $\hat{r}_{e d c}$ baseado no comportamento assintótico das funções $\log L_{n, k}$ e da sequência $c_{n}$. Esse resultado foi estabelecido em contextos particulares, diretamente ou indiretamente, por diversos pesquisadores, dentre eles Nishii (1988), Finesso (1990) e Dorea (2008).

Teorema 1.5. Seja $\mathbb{X}$ um processo estocástico a tempo discreto com valores em $\mathbb{R}^{m}, \mathbb{M}$ sua respectiva sequência de modelos aninhados, $m_{r} \in \bigcup_{k=0}^{\infty} M_{k}$ de ordem $r, \hat{r}_{\text {edc }}$ como definido em (1.5), $\hat{\theta}_{k}$ e $\log L_{n}\left(\hat{\theta}_{k}\right)$ como na Definição 1.3.

(i) $\hat{r}_{\text {edc }}$ é fortemente consistente $\left(\hat{r}_{\text {edc }} \underset{\text { q.c. }}{\rightarrow} r\right)$ se

(H1) para $k<r$, existe $c_{1} \in(0, \infty)$ tal que

$$
\lim _{n \rightarrow \infty} \frac{\log L_{n}\left(\hat{\theta}_{r}\right)-\log L_{n}\left(\hat{\theta}_{k}\right)}{n} \geq c_{1} q . c .,
$$

(H2) para $k>r$, existe $c_{2} \in(0, \infty)$ tal que

$$
\limsup _{n \rightarrow \infty} \frac{\log L_{n}\left(\hat{\theta}_{k}\right)-\log L_{n}\left(\hat{\theta}_{r}\right)}{\log \log n} \leq c_{2}(\gamma(k)-\gamma(r)) \text { q.c. }
$$


e $c_{n}$, dado em (1.5), satisfaz

$$
\lim _{n \rightarrow \infty} \frac{c_{n}}{n}=0 \quad e \quad \liminf _{n \rightarrow \infty} \frac{c_{n}}{\log \log n} \geq c_{2}
$$

(ii) $\hat{r}_{e d c}$ é consistente $\left(\hat{r}_{\text {edc }} \underset{P}{\rightarrow}\right.$ ) se Hl é satisfeita, $\lim _{n \rightarrow \infty} c_{n}=\infty, \lim _{n \rightarrow \infty} \frac{c_{n}}{n}=0 e$

(H3) para $k>r$

$$
\lim _{n \rightarrow \infty} \mathrm{P}\left(\log L_{n}\left(\hat{\theta}_{k}\right)-\log L_{n}\left(\hat{\theta}_{r}\right)>(\gamma(k)-\gamma(r)) c_{n}\right)=0 .
$$

(iii) $\hat{r}_{\text {edc }}$ é inconsistente se existe $c_{3} \in(0, \infty)$ tal que $\limsup _{n \rightarrow \infty} c_{n} \leq c_{3}<\infty e$ (H4) para $k \geq r$

$$
\lim _{n \rightarrow \infty} \mathrm{P}\left(\log L_{n}\left(\hat{\theta}_{k}\right)-\log L_{n}\left(\hat{\theta}_{r}\right)>c_{3}(\gamma(k)-\gamma(r))\right)>0 .
$$

Demonstração. Temos que

$$
\begin{aligned}
\left(-\log L_{n}\left(\hat{\theta}_{p}\right)+\gamma(p) c_{n}\right) & -\left(-\log L_{n}\left(\hat{\theta}_{l}\right)+\gamma(l) c_{n}\right) \\
& =\left(\log L_{n}\left(\hat{\theta}_{l}\right)-\log L_{n}\left(\hat{\theta}_{p}\right)\right)-c_{n}(\gamma(l)-\gamma(p)) .
\end{aligned}
$$

(i) Usando a hipótese $\mathrm{H} 2$ e (1.6), substituindo $p=r$ e $l=k>r$ em (1.7), temos

$$
\begin{aligned}
\limsup _{n \rightarrow \infty} \frac{\left(-\log L_{n}\left(\hat{\theta}_{r}\right)+\gamma(r) c_{n}\right)-\left(-\log L_{n}\left(\hat{\theta}_{k}\right)+\gamma(k) c_{n}\right)}{\log \log n} & \leq c_{2}(\gamma(k)-\gamma(r))-\liminf _{n \rightarrow \infty}\left(\frac{c_{n}}{\log \log n}\right)(\gamma(k)-\gamma(r)) \quad \text { q.c. } \\
& \leq c_{2}(\gamma(k)-\gamma(r))-c_{2}(\gamma(k)-\gamma(r)) \quad \text { q.c. } \\
& =0 .
\end{aligned}
$$

Da mesma forma, se $l=r$ e $p=k<r$, em (1.7), usando a hipótese H1 e (1.6), temos q.c.

$$
\begin{aligned}
\liminf _{n \rightarrow \infty} \frac{\left(-\log L_{n}\left(\hat{\theta}_{k}\right)+\gamma(k) c_{n}\right)-\left(-\log L_{n}\left(\hat{\theta}_{r}\right)+\gamma(r) c_{n}\right)}{n} & \geq c_{1}-\limsup _{n \rightarrow \infty} \frac{c_{n}}{n}(\gamma(r)-\gamma(k)) \quad q . c . \\
& >0 \text { q.c. }
\end{aligned}
$$

Então $\hat{r}_{e d c} \underset{q . c .}{\rightarrow} r$.

(ii) Usando H1 concluímos (1.8) e portanto $\lim _{n \rightarrow \infty} \hat{r}_{e d c} \geq r$ q.c. Por outro lado temos que

$$
\mathrm{P}\left(\hat{r}_{e d c}>r\right) \leq \sum_{k=r+1}^{K} \mathrm{P}(\operatorname{EDC}(k)<\operatorname{EDC}(r))
$$


e para $k>r$, usando (1.7) $\operatorname{com} p=r$ e $l=k$,

$$
\begin{aligned}
\mathrm{P}(\operatorname{EDC}(k)<\operatorname{EDC}(r)) & =\mathrm{P}\left(-\log L_{n}\left(\hat{\theta}_{k}\right)+\gamma(k) c_{n}<-\log L_{n}\left(\hat{\theta}_{r}\right)+\gamma(r) c_{n}\right) \\
& =\mathrm{P}\left(\log L_{n}\left(\hat{\theta}_{k}\right)-\log L_{n}\left(\hat{\theta}_{r}\right)>c_{n}(\gamma(k)-\gamma(r))\right)
\end{aligned}
$$

Usando H3 e (1.9) temos que $\hat{r}_{e d c} \underset{P}{\rightarrow} r$.

(iii) Para $k>r$,

$$
\lim _{n \rightarrow \infty} \mathrm{P}\left(\hat{r}_{e d c}>r\right)>\lim _{n \rightarrow \infty} \mathrm{P}(\mathrm{EDC}(r)-\operatorname{EDC}(k)>0)
$$

e tomando $l=k>r$ e $p=r$ em (1.7) temos

$$
\begin{aligned}
\lim _{n \rightarrow \infty} \mathrm{P}(\operatorname{EDC}(r)-\operatorname{EDC}(k)>0) & =\lim _{n \rightarrow \infty} \mathrm{P}\left(\log L_{n}\left(\hat{\theta}_{k}\right)-\log L_{n}\left(\hat{\theta}_{r}\right)>c_{n}(\gamma(k)-\gamma(r))\right) \\
& \geq \lim _{n \rightarrow \infty} \mathrm{P}\left(\log L_{n}\left(\hat{\theta}_{k}\right)-\log L_{n}\left(\hat{\theta}_{r}\right)>c_{3}(\gamma(k)-\gamma(r))\right) \\
& >0 .
\end{aligned}
$$

Portanto $\hat{r}_{e d c}$ possui uma probabilidade positiva a superestimar $r$.

Se $\log L_{n, k}$ é a $\log$-verossimilhança, temos que a diferença $\log L_{n}\left(\hat{\theta}_{k}\right)-\log L_{n}\left(\hat{\theta}_{r}\right)$ é o logaritmo da razão da verossimilhança de Neyman-Pearson, que foi bastante utilizada nos trabalhos focados em testes de hipóteses, anteriores a Akaike (1974). Portanto, é comum se obter na literatura o comportamento assintótico dessa diferença para cada caso, que geralmente converge para uma distribuição qui-quadrado (Whittle 1951, Anderson \& Goodman 1957, van der Vaart 2000, Shao 2007). Com esse resultado temos condições de provar as hipóteses $\mathrm{H} 3$ e H4. A hipótese $\mathrm{H} 1$ geralmente pode ser demonstrada, sem muitas dificuldades, usando a Lei Forte dos Grandes Números e/ou divergência de Kullback-Leibler (Kullback 1959). A questão maior é a demonstração da hipótese H2, que geralmente depende da Lei do Logaritmo Iterado, que nem sempre está disponível de forma utilizável para o processo a que se pretende estender o EDC.

Nesse sentido, o Teorema 1.6 conclui $\mathrm{H} 2$ usando hipóteses sobre o comportamento assintótico do estimador $\hat{\theta}_{k}$ ou sobre o comportamento das derivadas de primeira ordem das funções $\log L_{n, k}$. Para isso, são necessárias as condições de regularidade abaixo. Observa-se que Nishii (1988) usa condições e resultado semelhante para o caso i.i.d. 
Condição 1.1 (Regularidade). Seja $\mathbb{X}=\left\{X_{t}\right\}_{t \in \mathbb{N}}$ um processo estocástico a tempo discreto com valores em $\mathbb{R}^{m}, \mathbb{M}$ sua respectiva sequência de modelos aninhados, $L_{n, k}$ como na Definição 1.3, $m_{r}=f\left(x_{1}^{n}, \theta_{r}\right) \in M_{r}$ as densidades de dimensão finita de $\mathbb{X}, k \geq r e \hat{\theta}_{k} o$ estimador de máxima verossimilhança de $\theta_{r}$. São satisfeitos os seguintes.

(i) $\theta_{r}$ é ponto interior de $\Theta_{k} e$

$$
\hat{\theta}_{k} \underset{q . c .}{\rightarrow} \theta_{r}
$$

(ii) Para todo $k, n \in \mathbb{N}, \log L_{n, k}(\theta)$ e suas derivadas, $D_{\theta}^{1}\left(\log L_{n, k}(\theta)\right), D_{\theta}^{2}\left(\log L_{n, k}(\theta)\right) e$ $D_{\theta}^{3}\left(\log L_{n, k}(\theta)\right)$, são mensuráveis com respeito a $x_{1}^{n}$ e contínuas com respeito a $\theta$.

(iii) Para $\dot{\theta}=(1-s) \hat{\theta}_{k}+s \theta_{r}, s \in(0,1),(i, j, l) \in\{1, \ldots, \gamma(k)\}^{3}$,

$$
\lim _{n \rightarrow \infty} \frac{\left(D_{\theta}^{3}\left(\log L_{n, k}(\dot{\theta})\right)\right)_{i, j, l}}{n}<\infty \quad \text { q.c. }
$$

Teorema 1.6. Seja $\mathbb{X}$ um processo estocástico a tempo discreto com valores em $\mathbb{R}^{m}, \mathbb{M}$ sua respectiva sequência de modelos aninhados, $\log L_{n}\left(\hat{\theta}_{k}\right)$ como na Definição 1.3, $\theta_{r}=$ $\left(\alpha_{1}, \ldots, \alpha_{\gamma(k)}\right) \in \Theta_{k}, \hat{\theta}_{k}=\left(\hat{\alpha}_{1}, \ldots, \hat{\alpha}_{\gamma(k)}\right) \in \Theta_{k}, \hat{\theta}_{k}$ o estimador de máxima verossimilhança de $\theta_{r}$ e valem as Condições de Regularidade (Condição 1.1).

(i) Se existe matriz $A_{2}$ positiva definida tal que

$$
\lim _{n \rightarrow \infty}-\frac{D_{\theta}^{2}\left(\log L_{n}\left(\hat{\theta}_{k}\right)\right)}{n}=A_{2} \quad \text { q.c. }
$$

e existe $c_{4} \in(0, \infty)$ tal que

$$
\limsup _{n \rightarrow \infty}\left\|\frac{\sqrt{n}\left(\theta_{r}-\hat{\theta}_{k}\right)}{\sqrt{2 \log \log n}}\right\| \leq c_{4} \quad \text { q.c. }
$$

então

$$
\limsup _{n \rightarrow \infty} \frac{\log L_{n}\left(\hat{\theta}_{k}\right)-\log L_{n}\left(\hat{\theta}_{r}\right)}{\log \log n} \leq 2 c_{4}^{2} \lambda_{1} \quad \text { q.c. }
$$

onde $\lambda_{1}$ é o maior autovalor de $A_{2}$.

(ii) Se existe matriz $A_{2}$ positiva definida tal que, para $\dot{\theta}=s \theta_{r}+(1-s) \hat{\theta}_{k}$ e $s \in[0,1]$,

$$
\lim _{n \rightarrow \infty}-\frac{D_{\theta}^{2}\left(\log L_{n}(\dot{\theta})\right)}{n}=A_{2} \quad \text { q.c. },
$$


e existe $c_{5} \in(0, \infty)$ tal que

$$
\limsup _{n \rightarrow \infty}\left\|\frac{D_{\theta}^{1} \log L_{n}\left(\theta_{r}\right)}{\sqrt{2 n \log \log n}}\right\| \leq c_{5} \quad \text { q.c. }
$$

então, para todo $i \in\{1, \ldots, \gamma(k)\}$,

$$
\limsup _{n \rightarrow \infty}\left|\frac{\sqrt{n}\left(\hat{\alpha}_{i}-\alpha_{i}\right)}{\sqrt{2 \log \log n}}\right|<\infty \quad \text { q.c. }
$$

$e$

$$
\limsup _{n \rightarrow \infty} \frac{\log L_{n}\left(\hat{\theta}_{k}\right)-\log L_{n}\left(\hat{\theta}_{r}\right)}{\log \log n} \leq \frac{2 c_{5}^{2}}{\lambda_{\gamma(k)}} \quad \text { q.c. }
$$

onde $\lambda_{\gamma(k)}$ é o menor autovalor de $A_{2}$.

(iii) Se para todo $(i, j) \in\{1, \ldots, \gamma(k)\}^{2}$, existem $c_{6}, c_{7}$ tais que

$$
\limsup _{n \rightarrow \infty}\left|\frac{\sqrt{n}\left(\hat{\alpha}_{i}-\alpha_{i}\right)}{\sqrt{2 \log \log n}}\right| \leq c_{6} \quad \text { q.c. }
$$

$e$

$$
\lim _{n \rightarrow \infty}\left(\frac{D_{\theta}^{2}\left(\log L_{n}\left(\hat{\theta}_{k}\right)\right)}{n}\right)_{i, j} \leq c_{7} \quad q . c .,
$$

então

$$
\limsup _{n \rightarrow \infty} \frac{\log L_{n}\left(\hat{\theta}_{k}\right)-\log L_{n}\left(\hat{\theta}_{r}\right)}{\log \log n} \leq 2 \gamma(k)^{2} c_{6}^{2} c_{7} \quad \text { q.c. }
$$

Demonstração. Usando as condições de regularidade e tomando a expansão em série de Taylor para $\log L_{n, k}\left(\theta_{r}\right)$ no ponto $\hat{\theta}_{k}$ temos

$$
\begin{aligned}
\log L_{n, k}\left(\theta_{r}\right)= & \log L_{n, k}\left(\hat{\theta}_{k}\right)+\left(\theta_{r}-\hat{\theta}_{k}\right) D_{\theta}^{1}\left(\log L_{n, k}\left(\hat{\theta}_{k}\right)\right) \\
& +\frac{1}{2}\left(\theta_{r}-\hat{\theta}_{k}\right) D_{\theta}^{2}\left(\log L_{n, k}\left(\hat{\theta}_{k}\right)\right)\left(\theta_{r}-\hat{\theta}_{k}\right)^{T}+r_{n}\left(\theta_{r}-\hat{\theta}_{k}\right)
\end{aligned}
$$

onde

$$
r_{n}\left(\theta_{r}-\hat{\theta}_{k}\right)=\frac{1}{3 !} \sum_{i, j, l}\left(D_{\theta}^{3} \log L_{n, k}(\dot{\theta})\right)_{i, j, l}\left(\alpha_{i}-\hat{\alpha}_{i}\right)\left(\alpha_{j}-\hat{\alpha}_{j}\right)\left(\alpha_{l}-\hat{\alpha}_{l}\right)
$$

$\theta_{r}=\left(\alpha_{i}, \ldots, \alpha_{\gamma(k)}\right), \hat{\theta}_{k}=\left(\hat{\alpha}_{i}, \ldots, \hat{\alpha}_{\gamma(k)}\right)$ e $\dot{\theta}=(1-s) \hat{\theta}_{k}+s \theta_{r}, s \in(0,1)$. Como $\hat{\theta}_{k}$ maximiza 
$L_{n, k}$, temos que $D_{\theta}^{1}\left(\log L_{n, k}\left(\hat{\theta}_{k}\right)\right)=0$. Organizando (1.19) e dividindo por $\log \log n$, temos

$$
\begin{aligned}
\limsup _{n \rightarrow \infty} \frac{\log L_{n, k}\left(\hat{\theta}_{k}\right)-\log L_{n, k}\left(\theta_{r}\right)}{\log \log n} \leq & \limsup _{n \rightarrow \infty} \frac{\sqrt{n}\left(\theta_{r}-\hat{\theta}_{k}\right)}{\sqrt{2 \log \log n}} \frac{-D_{\theta}^{2}\left(\log L_{n, k}\left(\hat{\theta}_{k}\right)\right)}{n} \frac{\left(\sqrt{n}\left(\theta_{r}-\hat{\theta}_{k}\right)\right)^{T}}{\sqrt{2 \log \log n}} \\
& +\limsup _{n \rightarrow \infty} \frac{\left|r_{n}\left(\theta_{r}-\hat{\theta}_{k}\right)\right|}{\log \log n}
\end{aligned}
$$

Usando (1.13) ou (1.26) ou (1.17) temos que

$$
\lim _{n \rightarrow \infty}\left|\frac{\sqrt{n}\left(\alpha_{i}-\hat{\alpha}_{i}\right)}{\sqrt{\log \log n}}\right|<\infty \quad \text { q.c. }
$$

Portanto, usando (1.11) e (1.10),

$$
\begin{aligned}
\limsup _{n \rightarrow \infty} \frac{\left|r_{n}\left(\theta_{r}-\hat{\theta}_{k}\right)\right|}{\log \log n} & \leq \limsup _{n \rightarrow \infty} \frac{1}{3 !} \sum_{i, j, l}\left|\frac{\left(D_{\theta}^{3} \log L_{n, k}(\dot{\theta})\right)_{i, j, l}}{n}\right|\left|\frac{\sqrt{n}\left(\alpha_{i}-\hat{\alpha}_{i}\right)}{\sqrt{\log \log n}}\right|\left|\frac{\sqrt{n}\left(\alpha_{j}-\hat{\alpha}_{j}\right)}{\sqrt{\log \log n}}\right|\left|\alpha_{l}-\hat{\alpha}_{l}\right| \\
& \leq c \sum_{l} \limsup _{n \rightarrow \infty}\left|\alpha_{l}-\hat{\alpha}_{l}\right|=0 \quad q . c .
\end{aligned}
$$

(i) Como $A_{2}$ é positiva definida, temos que $\forall x \in \mathbb{R}^{\gamma(k)}, x A_{2} x^{T} \leq \lambda_{1}\|x\|^{2}$, para $\lambda_{1}$ o maior autovalor de $A_{2}$ (Zhang 2011). Usando (1.20), (1.12), (1.13) e (1.21) temos

$$
\begin{aligned}
\limsup _{n \rightarrow \infty} \frac{\log L_{n, k}\left(\hat{\theta}_{k}\right)-\log L_{n, k}\left(\theta_{r}\right)}{\log \log n} & \leq \limsup _{n \rightarrow \infty} \frac{\sqrt{n}\left(\theta_{r}-\hat{\theta}_{k}\right)}{\sqrt{2 \log \log n}} \frac{-D_{\theta}^{2}\left(\log L_{n, k}\left(\hat{\theta}_{k}\right)\right)}{n} \frac{\left(\sqrt{n}\left(\theta_{r}-\hat{\theta}_{k}\right)\right)^{T}}{\sqrt{2 \log \log n}} \\
& \leq \limsup _{n \rightarrow \infty} \lambda_{1}\left\|\frac{\sqrt{n}\left(\theta_{r}-\hat{\theta}_{k}\right)}{\sqrt{2 \log \log n}}\right\|^{2} \\
& \leq \lambda_{1} c_{4}^{2} \text { q.c. }
\end{aligned}
$$

Portanto, aplicando (1.22) duas vezes e usando (1.4), obtemos

$$
\begin{aligned}
\limsup _{n \rightarrow \infty} \frac{\log L_{n, k}\left(\hat{\theta}_{k}\right)-\log L_{n, r}\left(\hat{\theta}_{r}\right)}{\log \log n} & =\limsup _{n \rightarrow \infty} \frac{\log L_{n, k}\left(\hat{\theta}_{k}\right)-\log L_{n, k}\left(\theta_{r}\right)-\left(\log L_{n, r}\left(\hat{\theta}_{r}\right)-\log L_{n, r}\left(\theta_{r}\right)\right)+\left(\log L_{n, k}\left(\theta_{r}\right)-\log L_{n, r}\left(\theta_{r}\right)\right)}{\log \log n} \\
& \leq \limsup _{n \rightarrow \infty} \frac{\log L_{n, k}\left(\hat{\theta}_{k}\right)-\log L_{n, k}\left(\theta_{r}\right)}{\log \log n}+\limsup _{n \rightarrow \infty} \frac{\log L_{n, r}\left(\hat{\theta}_{r}\right)-\log L_{n, r}\left(\theta_{r}\right)}{\log \log n} \\
& \leq 2 \lambda_{1} c_{4}^{2} .
\end{aligned}
$$

Usamos em (1.23) que, pela definição de $L_{n, k}, \log L_{n, k}\left(\hat{\theta}_{k}\right) \geq \log L_{n, k}\left(\theta_{r}\right)$ e $\log L_{n, r}\left(\hat{\theta}_{r}\right) \geq$ $\log L_{n, r}\left(\theta_{r}\right)$.

(ii) Tomando a série de Taylor de $D_{\theta}^{1} \log L_{n, k}\left(\hat{\theta}_{k}\right)$ no ponto $\theta_{r}$,

$$
\begin{aligned}
(0, \cdots, 0) & =D_{\theta}^{1} \log L_{n, k}\left(\hat{\theta}_{k}\right) \\
& =D_{\theta}^{1} \log L_{n, k}\left(\theta_{r}\right)+\left(\hat{\theta}_{k}-\theta_{r}\right) D_{\theta}^{2} \log L_{n, k}(\dot{\theta})
\end{aligned}
$$


onde $\dot{\theta}=s \theta_{r}+(1-s) \hat{\theta}_{k}$ e $s \in(0,1)$. Organizando,

$$
\begin{aligned}
\frac{1}{\sqrt{n \log \log n}} D_{\theta}^{1} \log L_{n, k}\left(\theta_{r}\right) & =\frac{\sqrt{n}}{n \sqrt{\log \log n}}\left\{\left(\hat{\theta}_{k}-\theta_{r}\right) D_{\theta}^{2} \log L_{n, k}(\dot{\theta})\right\} \\
& =-\frac{\sqrt{n}}{\sqrt{\log \log n}}\left(\hat{\theta}_{k}-\theta_{r}\right)\left[\frac{D_{\theta}^{2} \log L_{n, k}(\dot{\theta})}{n}\right] .
\end{aligned}
$$

Como $A_{2}$ é positiva definida, é invertível, e para $n$ suficientemente grande,

$$
A_{n}:=-\left[\frac{D_{\theta}^{2} \log L_{n, k}(\dot{\theta})}{n}\right]
$$

possui inversa $A_{n}^{-1}$, então

$$
\frac{1}{\sqrt{n \log \log n}} D_{\theta}^{1} \log L_{n, k}\left(\theta_{r}\right) A_{n}^{-1}=\frac{\sqrt{n}}{\sqrt{\log \log n}}\left(\hat{\theta}_{k}-\theta_{r}\right) .
$$

Aplicando (1.24), (1.21), (1.14), (1.15) e que $A_{n} \underset{\text { q.c. }}{\rightarrow} A_{2}$, obtemos

$$
\begin{aligned}
\limsup _{n \rightarrow \infty} \frac{\log L_{n, k}\left(\hat{\theta}_{k}\right)-\log L_{n, k}\left(\theta_{r}\right)}{\log \log n} & \leq \limsup _{n \rightarrow \infty} \frac{\left(D_{\theta}^{1} \log L_{n, k}\left(\theta_{r}\right)\right) A_{n}^{-1}}{\sqrt{2 n \log \log n}} \frac{-D_{\theta}^{2}\left(\log L_{n, k}\left(\hat{\theta}_{k}\right)\right)}{n}\left(\frac{\left(D_{\theta}^{1} \log L_{n, k}\left(\theta_{r}\right)\right) A_{n}^{-1}}{\sqrt{2 n \log \log n}}\right)^{T} \\
& =\limsup _{n \rightarrow \infty} \frac{D_{\theta}^{1} \log L_{n, k}\left(\theta_{r}\right)}{\sqrt{2 n \log \log n}} A_{2}^{-1} A_{2}\left(\frac{D_{\theta}^{1} \log L_{n, k}\left(\theta_{r}\right)}{\sqrt{2 n \log \log n}} A_{2}^{-1}\right)^{T} \\
& =\limsup _{n \rightarrow \infty} \frac{D_{\theta}^{1} \log L_{n, k}\left(\theta_{r}\right)}{\sqrt{2 n \log \log n}} A_{2}^{-1}\left(\frac{D_{\theta}^{1} \log L_{n, k}\left(\theta_{r}\right)}{\sqrt{2 n \log \log n}}\right)^{T} \\
& \leq \frac{1}{\lambda_{\gamma(k)}} \limsup _{n \rightarrow \infty}\left\|\frac{D_{\theta}^{1} \log L_{n, k}\left(\theta_{r}\right)}{\sqrt{2 n \log \log n}}\right\|^{2} \\
& \leq \frac{c_{5}^{2}}{\lambda_{\gamma(k)}} \text { q.c. }
\end{aligned}
$$

Usamos o fato de matrizes positivas definidas serem simétricas $\left(A_{2}=A_{2}^{T}\right)^{1} \mathrm{e}$ possuírem inversas positivas definidas. Aplicando o mesmo argumento de (1.23), concluímos de (1.25) a segunda inequação do resultado. Para a primeira, basta utilizar

\footnotetext{
${ }^{1}$ Nesse trabalho consideramos simetria na definição de matrizes positiva definidas.
} 
(1.24), considerar $P_{i}: \mathbb{R}^{\gamma(k)} \rightarrow \mathbb{R}$ como a projeção da coordenada $i$ e observar que

$$
\begin{aligned}
\limsup _{n \rightarrow \infty} \mid \frac{\sqrt{n}\left(\hat{\alpha}_{i}-\alpha_{i}\right)}{\sqrt{2 \log \log n} \mid} & =\limsup _{n \rightarrow \infty}\left|P_{i}\left(\frac{\sqrt{n}}{\sqrt{2 \log \log n}}\left(\hat{\theta}_{k}-\theta_{r}\right)\right)\right| \\
& =\limsup _{n \rightarrow \infty}\left|P_{i}\left(\frac{1}{\sqrt{2 n \log \log n}} D_{\theta}^{1} \log L_{n, k}\left(\theta_{r}\right) A_{n}^{-1}\right)\right| \\
& =\limsup _{n \rightarrow \infty}\left|P_{i}\left(\frac{1}{\sqrt{2 n \log \log n}} D_{\theta}^{1} \log L_{n, k}\left(\theta_{r}\right) A_{2}^{-1}\right)\right| \\
& <\infty .
\end{aligned}
$$

(iii) Aplicando (1.17), (1.18), (1.21) e (1.20), obtemos

$$
\limsup _{n \rightarrow \infty} \frac{\log L_{n}\left(\hat{\theta}_{k}\right)-\log L_{n}\left(\theta_{r}\right)}{\log \log n} \leq \gamma(k)^{2} c_{6}^{2} c_{7} \quad \text { q.c. }
$$

Com o mesmo raciocínio usado em (1.23), obtemos o resultado.

O resultado dos itens (i)-(iii) do Teorema 1.6 é basicamente o mesmo - uma cota superior em H2. Entretanto, quanto menor a cota superior encontrada maior é a classe de estimadores $\hat{r}_{e d c}$ fortemente consistentes. Assim, as três opções visam simplificar o trabalho de obtenção de uma cota pequena.

Geralmente, logo após a definição de determinado processo, a primeira questão abordada são as condições de ergodicidade para ser possível a aplicação dos equivalentes a Lei Forte dos Grandes Números (LFGN), Teorema do Limite Central (TLC) e Lei do Logaritmo Iterado (LLI). Na sequência, buscam-se condições para a consistência forte do estimador do parâmetro $\theta_{r}$ e a normalidade assintótica desse estimador. Para a obtenção desse último passo, geralmente são necessárias algumas das hipóteses exigidas pelo Teorema 1.6, que podem ser utilizadas para o estabelecimento dos estimadores EDC.

O item (ii) do Teorema 1.6, essencialmente, conclui H2 baseado na LLI para as derivadas de primeira ordem de $\log L_{n, k}$. Em resultado análogo, e usando técnica semelhante, Basawa \& Heyde (1976) conclui a normalidade assintótica de $\hat{\theta}_{r}$ a partir de normalidade assintótica das derivadas de primeira ordem da log-verossimilhança. Para isso são utilizadas condições semelhantes às exigidas pelo Teorema 1.6. 
Como $\hat{\theta}_{k}$ maximiza $\log L_{n, k}$, temos que a Hessiana no ponto $\hat{\theta}_{k}$ é negativa. Neste caso, $-D_{\theta}^{2} \log L_{n}\left(\hat{\theta}_{k}\right)$ é positiva para todo $n$. Suponha positiva definida para melhor entendimento da equação (1.12). A equação requer que $-D_{\theta}^{2} \log L_{n}\left(\hat{\theta}_{k}\right) / n$ possua limite quase certo a matriz $A_{2}$, positiva definida. Em outro ponto de vista, suponha que o processo seja markoviano e que $L_{n}$ seja a função verossimilhança, neste caso, suponha ainda que $-D_{\theta}^{2} \log L_{n}(\hat{\theta}) / n=-\sum D_{\theta}^{2} \log f_{\hat{\theta}}\left(x_{i} \mid x_{i-r}, \ldots, x_{i-1}\right) / n$ para alguma densidade condicional $f_{\hat{\theta}}\left(. \mid\right.$.). Se vale a LFGN para esse limite, temos que $-\sum D_{\theta}^{2} \log f_{\hat{\theta}}\left(x_{i} \mid x_{i-r}, \ldots, x_{i-1}\right) / n$ converge quase certamente para a matriz de Informação de Fisher para valores próximos a $\theta_{r}$ e, que por (1.12), deve ser positiva definida.

A normalidade assintótica do estimador $\hat{\theta}_{k}$ pode ser utilizada para concluir H3. O próximo resultado provê condições para isso.

Teorema 1.7. Seja $\mathbb{X}$ um processo estocástico a tempo discreto com valores em $\mathbb{R}^{m}, \mathbb{M}$ sua respectiva sequência de modelos aninhados, $L_{n}\left(\hat{\theta}_{k}\right)$ como na Definição 1.3, $\theta_{r}=\left(\alpha_{1}, \ldots, \alpha_{\gamma(k)}\right)$ $\in \Theta_{k}, \hat{\theta}_{k}=\left(\hat{\alpha}_{1}, \ldots, \hat{\alpha}_{\gamma(k)}\right) \in \Theta_{k}, \hat{\theta}_{k}$ o estimador de máxima verossimilhança de $\theta_{r}$ e valem as Condições de Regularidade (Condição 1.1). Se existe matriz $A_{2}$ positiva definida tal que

$$
\lim _{n \rightarrow \infty}-\frac{D_{\theta}^{2}\left(\log L_{n}\left(\hat{\theta}_{k}\right)\right)}{n}=A_{2} \quad \text { q.c. },
$$

para todo $i \in\{1, \ldots, \gamma(k)\}$,

$$
\limsup _{n \rightarrow \infty}\left|\frac{\sqrt{n}\left(\hat{\alpha}_{i}-\alpha_{i}\right)}{\sqrt{\log \log n}}\right|<\infty \quad \text { q.c. }
$$

e, para todo $h_{n}$, tal que $\lim _{n \rightarrow \infty} h_{n}=\infty$,

$$
\lim _{n \rightarrow \infty} P\left(\left\|\sqrt{n}\left(\theta_{r}-\hat{\theta}_{k}\right)\right\|>h_{n}\right)=0
$$

então

$$
\lim _{n \rightarrow \infty} \mathrm{P}\left(\log L_{n}\left(\hat{\theta}_{k}\right)-\log L_{n}\left(\hat{\theta}_{r}\right)>h_{n}\right)=0 .
$$

para todo $h_{n}$, tal que $\lim _{n \rightarrow \infty} h_{n}=\infty$.

Demonstração. Usando as condições de regularidade e tomando a expansão em série de 
Taylor para $\log L_{n, k}\left(\theta_{r}\right)$ no ponto $\hat{\theta}_{k}$ temos

$$
\begin{aligned}
\log L_{n, k}\left(\theta_{r}\right)= & \log L_{n, k}\left(\hat{\theta}_{k}\right)+\left(\theta_{r}-\hat{\theta}_{k}\right) D_{\theta}^{1}\left(\log L_{n, k}\left(\hat{\theta}_{k}\right)\right) \\
& +\frac{1}{2}\left(\theta_{r}-\hat{\theta}_{k}\right) D_{\theta}^{2}\left(\log L_{n, k}\left(\hat{\theta}_{k}\right)\right)\left(\theta_{r}-\hat{\theta}_{k}\right)^{T}+r_{n}\left(\theta_{r}-\hat{\theta}_{k}\right)
\end{aligned}
$$

onde

$$
r_{n}\left(\theta_{r}-\hat{\theta}_{k}\right)=\frac{1}{3 !} \sum_{i, j, l}\left(D_{\theta}^{3} \log L_{n, k}(\dot{\theta})\right)_{i, j, l}\left(\alpha_{i}-\hat{\alpha}_{i}\right)\left(\alpha_{j}-\hat{\alpha}_{j}\right)\left(\alpha_{l}-\hat{\alpha}_{l}\right)
$$

Para $\dot{\theta}=(1-s) \theta_{k}+s \theta_{r}, s \in(0,1)$. Usando (1.28) e (1.11), obtemos

$$
\begin{aligned}
\limsup _{n \rightarrow \infty}\left|r_{n}\left(\theta_{r}-\hat{\theta}_{k}\right)\right| & \leq \limsup _{n \rightarrow \infty} \frac{1}{3 !} \sum_{i, j, l}\left|\frac{\left(D_{\theta}^{3} \log L_{n, k}(\dot{\theta})\right)_{i, j, l}}{n}\right|\left|\frac{\sqrt{n}\left(\alpha_{i}-\hat{\alpha}_{i}\right)}{\sqrt{\log \log n}}\right|\left|\frac{\sqrt{n}\left(\alpha_{j}-\hat{\alpha}_{j}\right)}{\sqrt{\log \log n}}\right|\left|\frac{\sqrt{n}\left(\alpha_{l}-\hat{\alpha}_{l}\right)}{\sqrt{\log \log n}}\right| \frac{(\log \log n)^{3 / 2}}{\sqrt{n}} \\
& \leq c \operatorname{cimsup}_{n \rightarrow \infty} \frac{(\log \log n)^{3 / 2}}{\sqrt{n}}=0 \quad \text { q.c. }
\end{aligned}
$$

Usando (1.27), para $n$ suficientemente grande, $A_{n}=-\frac{D_{\theta}^{2}\left(\log L_{n, k}\left(\hat{\theta}_{k}\right)\right)}{n}$ é positiva definida, e portanto possui maior autovalor, $\lambda_{n}$, tal que $\lambda_{n} \underset{\text { q.c. }}{\rightarrow} \lambda_{1}$, onde $\lambda_{1}^{n}$ é o maior autovalor de $A_{2}$. Como $\forall x, x A_{n} x^{T} \leq \lambda_{n}\|x\|^{2}$, temos

$$
\begin{aligned}
\lim _{n \rightarrow \infty} \mathrm{P}\left(\log L_{n, k}\left(\hat{\theta}_{k}\right)-\log L_{n, k}\left(\theta_{r}\right)>h_{n}\right) & =\lim _{n \rightarrow \infty} \mathrm{P}\left(\frac{1}{2} \sqrt{n}\left(\theta_{r}-\hat{\theta}_{k}\right) \frac{-D_{\theta}^{2}\left(\log L_{n}\left(\hat{\theta}_{k}\right)\right)}{n}\left(\sqrt{n}\left(\theta_{r}-\hat{\theta}_{k}\right)\right)^{T}>h_{n}\right) \\
& \leq \lim _{n \rightarrow \infty} \mathrm{P}\left(\frac{\lambda_{n}}{2}\left\|\sqrt{n}\left(\theta_{r}-\hat{\theta}_{k}\right)\right\|^{2}>h_{n}\right) \\
& =\lim _{n \rightarrow \infty} \mathrm{P}\left(\left\|\sqrt{n}\left(\theta_{r}-\hat{\theta}_{k}\right)\right\|>\frac{\sqrt{2 h_{n}}}{\sqrt{\lambda_{n}}}\right)=0
\end{aligned}
$$

onde concluímos (1.29), considerando (1.4).

Em resumo, se as condições abaixo são atendidas, o Teorema 1.6 pode ser utilizado para obter H2. Adicionalmente, se a hipótese H1 é verdadeira, as condições para a definição de uma classe de estimadores EDC fortemente consistentes são atendidas.

(i) $\theta_{r}$ é ponto interior de $\Theta_{k} \mathrm{e}$

$$
\hat{\theta}_{k} \underset{q . c .}{\rightarrow} \theta_{r}
$$

(ii) Para todo $k, n \in \mathbb{N}, \log L_{n, k}(\theta)$ e suas derivadas, $D_{\theta}^{1}\left(\log L_{n, k}(\theta)\right), D_{\theta}^{2}\left(\log L_{n, k}(\theta)\right)$ e $D_{\theta}^{3}\left(\log L_{n, k}(\theta)\right)$, são mensuráveis com respeito a $x_{1}^{n}$ e contínuas com respeito a $\theta$. 
(iii) Para $\dot{\theta}=(1-s) \hat{\theta}_{k}+s \theta_{r}, s \in(0,1),(i, j, l) \in\{1, \ldots, \gamma(k)\}^{3}$,

$$
\lim _{n \rightarrow \infty} \frac{\left(D_{\theta}^{3}\left(\log L_{n, k}(\dot{\theta})\right)\right)_{i, j, l}}{n}<\infty \quad \text { q.c. }
$$

(iv) Existe matriz $A_{2}$ positiva definida tal que, para $\dot{\theta}=s \theta_{r}+(1-s) \hat{\theta}_{k}$ e $s \in[0,1]$,

$$
\lim _{n \rightarrow \infty}-\frac{D_{\theta}^{2}\left(\log L_{n, k}(\dot{\theta})\right)}{n}=A_{2} \quad \text { q.c. }
$$

(v)

$$
\limsup _{n \rightarrow \infty}\left\|\frac{D_{\theta}^{1} \log L_{n, k}\left(\theta_{r}\right)}{\sqrt{2 n \log \log n}}\right\| \leq c_{5} \quad \text { q.c. }
$$

\subsection{Generalização: ordem em modelos parcialmente ani- nhados}

Sob algumas hipóteses, um processo $\mathrm{AR}(r)$ pode ser especificado com $r$ parâmetros e um processo $\mathrm{AR}(r-1)$ pode ser considerado como um processo $\mathrm{AR}(r)$ com um parâmetro pré-fixado. Nesse sentido temos o conceito de "aninhamento" definido na Seção anterior e podemos considerar a sequência de processos $\{A R(0), A R(1), \cdots\}$ como uma sequência de modelos aninhados. De forma semelhante, temos os processos $\operatorname{ARMA}\left(r_{1}, r_{2}\right)$, que são especificados com $r_{1}+r_{2}$ parâmetros. Quando $k_{1} \geq r_{1}$ e $k_{2} \geq r_{2}$ podemos fixar alguns parâmetros e considerar um modelo $\operatorname{ARMA}\left(r_{1}, r_{2}\right)$ imerso em um modelo $\operatorname{ARMA}\left(k_{1}, k_{2}\right)$. Entretanto quando $k_{1}<r_{1}$ e $k_{2} \geq r_{2}$ o processo de imersão não é aplicável.

Mesmo nesses casos, os critérios de informação (AIC, BIC, HQC, dentre outros) são utilizados na determinação da ordem, como exemplo cita-se Hannan (1980). Entretanto, como os problemas de determinação de ordem são tratados na literatura de forma particularizada, a questão de aninhamento não é abordada diretamente. Por outro lado, para se propor uma generalização, há a necessidade de se definir bem esses conceitos no contexto de seleção de modelos.

A alternativa aparentemente viável seria definir o aninhamento diagonal, como exemplo, para o caso mencionado teríamos a sequência de modelos aninhados $\{A R M A(0,0)$, $\operatorname{ARMA}(1,1), \cdots\}$, e tratar o caso geral fixando uma dimensão e considerando sequências de 
modelos aninhados na outra dimensão. Entretanto, para implementar essa alternativa seria necessário dividir o processo de estimação da ordem em etapas, o que não é desejável.

Para o caso unidimensional, a relação de aninhamento na sequência de modelos aninhado coincide com a relação de ordem em $\mathbb{N}$. Assim, a definição proposta nesse trabalho para tratar casos com ordem de dimensão $p$ é relacionar a ordem parcial em $\mathbb{N}^{p}$ com a relação de aninhamento. As próximas definições tratam essa generalização. Em seguida são apresentados os resultados da Seção anterior nesse novo contexto.

Definição 1.8. Para $p \in \mathbb{N}$, seja $k=\left(k_{1}, \ldots, k_{p}\right) \in \mathbb{N}^{p}$ e $r=\left(r_{1}, \ldots, r_{p}\right) \in \mathbb{N}^{p}$. Define-se os seguintes.

(i) A relação de ordem: $k \geq r \Leftrightarrow k_{i} \geq r_{i}$ para $i=1 \ldots p$. [Com isso $\left(\mathbb{N}^{p}, \geq\right)$ é um conjunto parcialmente ordenado.]

(ii) $r \leq k$ quando $k \geq r$ e $k<r$ quando $r \geq k e k \neq r$.

(iii) $k \nsupseteq r$ se $r<k$ ou quando $k$ e $r$ não estão relacionados.

Definição 1.9. (i) Para $p \in \mathbb{N}, \mathbb{M}=\left\{M_{k}\right\}_{k \in \mathbb{N} p}$ é uma classe de modelos parcialmente aninhados se vale: $k \leq r$ se e somente se $M_{k} \subseteq M_{r}$.

(ii) Se $\mathbb{M}$ é classe de modelos parcialmente aninhados e $m_{r} \in \bigcup_{k \in \mathbb{N} p} M_{k}$, dizemos que $m_{r}$ é de ordem $r$ se $m_{r} \in M_{r}$ e, se $m_{r} \in M_{j}$, então $M_{r} \subseteq M_{j}$.

(iii) Se $\mathbb{M}=\left\{M_{k}\right\}_{k \in \mathbb{N}^{p}}$ é classe de modelos parcialmente aninhados, denotamos $\gamma(k)=$ $\operatorname{dim}\left(\Theta_{k}\right)$.

Com pequenas adaptações nos Teoremas 1.5, 1.6 e 1.7 obtemos os seguintes resultados.

Teorema 1.10. Seja $\mathbb{X}$ um processo estocástico a tempo discreto com valores em $\mathbb{R}^{m}, \mathbb{M}$ sua respectiva classe de modelos parcialmente aninhados, $m_{r} \in \bigcup_{k \in \mathbb{N} p} M_{k}$ de ordem $r$ e $\hat{r}_{\text {edc }}$ como definido em (1.5).

(i) $\hat{r}_{\text {edc }}$ éfortemente consistente $\left(\hat{r}_{\text {edc }} \underset{\text { q.c. }}{\rightarrow} r\right)$ se 
(H1) para $k \nsupseteq r$, existe $c_{1} \in(0, \infty)$ tal que

$$
\lim _{n \rightarrow \infty} \frac{\log L_{n}\left(\hat{\theta}_{r}\right)-\log L_{n}\left(\hat{\theta}_{k}\right)}{n} \geq c_{1} q . c .
$$

(H2) para $k>r$, existe $c_{2} \in(0, \infty)$ tal que

$$
\limsup _{n \rightarrow \infty} \frac{\log L_{n}\left(\hat{\theta}_{k}\right)-\log L_{n}\left(\hat{\theta}_{r}\right)}{\log \log n} \leq c_{2}(\gamma(k)-\gamma(r)) \text { q.c. }
$$

e $c_{n}$ satisfaz

$$
\lim _{n \rightarrow \infty} \frac{c_{n}}{n}=0 \quad e \quad \liminf _{n \rightarrow \infty} \frac{c_{n}}{\log \log n} \geq c_{2}
$$

(ii) $\hat{r}_{e d c}$ é consistente $\left(\hat{r}_{e d c} \underset{P}{\rightarrow}\right.$ ) se H1 é satisfeita, $\lim _{n \rightarrow \infty} c_{n}=\infty, \lim _{n \rightarrow \infty} \frac{c_{n}}{n}=0 e$ (H3) para $k>r$

$$
\lim _{n \rightarrow \infty} \mathrm{P}\left(\log L_{n}\left(\hat{\theta}_{k}\right)-\log L_{n}\left(\hat{\theta}_{r}\right)>(\gamma(k)-\gamma(r)) c_{n}\right)=0
$$

(iii) $\hat{r}_{e d c}$ é inconsistente se $\limsup _{n \rightarrow \infty} c_{n} \leq c_{3}<\infty e$

(H4) para $k \geq r$

$$
\lim _{n \rightarrow \infty} \mathrm{P}\left(\log L_{n}\left(\hat{\theta}_{k}\right)-\log L_{n}\left(\hat{\theta}_{r}\right)>c_{3}(\gamma(k)-\gamma(r))\right)>0 .
$$

Condição 1.2 (Regularidade). Seja $\mathbb{X}=\left\{X_{t}\right\}_{t \in \mathbb{N}}$ um processo estocástico a tempo discreto com valores em $\mathbb{R}^{m}$, M sua respectiva classe de modelos parcialmente aninhados, $L_{n, k}$ como na Definição 1.3, $m_{r}=f\left(x_{1}^{n}, \theta_{r}\right) \in M_{r}$ as densidades de dimensão finita de $\mathbb{X}, k \geq r$ e $\hat{\theta}_{k} o$ estimador de máxima verossimilhança de $\theta_{r}$. São satisfeitos os seguintes.

(i) $\theta_{r}$ é ponto interior de $\Theta_{k} e$

$$
\hat{\theta}_{k} \underset{q . c .}{\rightarrow} \theta_{r}
$$

(ii) Para todo $k, n \in \mathbb{N}, \log L_{n, k}(\theta)$ e suas derivadas, $D_{\theta}^{1}\left(\log L_{n, k}(\theta)\right), D_{\theta}^{2}\left(\log L_{n, k}(\theta)\right) e$ $D_{\theta}^{3}\left(\log L_{n, k}(\theta)\right)$, são mensuráveis com respeito a $x_{1}^{n}$ e contínuas com respeito a $\theta$.

(iii) Para $\dot{\theta}=(1-s) \hat{\theta}_{k}+s \theta_{r}, s \in(0,1),(i, j, l) \in\{1, \ldots, \gamma(k)\}^{3}$,

$$
\lim _{n \rightarrow \infty} \frac{\left(D_{\theta}^{3}\left(\log L_{n, k}(\dot{\theta})\right)\right)_{i, j, l}}{n}<\infty \quad \text { q.c. }
$$


Teorema 1.11. Seja $\mathbb{X}$ um processo estocástico a tempo discreto com valores em $\mathbb{R}^{m}, \mathbb{M}$ sua respectiva classe de modelos parcialmente aninhados, $L_{n}\left(\hat{\theta}_{k}\right)$ como na Definição 1.3, $\theta_{r}=$ $\left(\alpha_{1}, \ldots, \alpha_{\gamma(k)}\right) \in \Theta_{k}, \hat{\theta}_{k}=\left(\hat{\alpha}_{1}, \ldots, \hat{\alpha}_{\gamma(k)}\right) \in \Theta_{k}, \hat{\theta}_{k}$ o estimador de máxima verossimilhança de $\theta_{r}$ e valem as Condições de Regularidade (Condição 1.2).

(i) Se existe matriz $A_{2}$ positiva definida tal que

$$
\lim _{n \rightarrow \infty}-\frac{D_{\theta}^{2}\left(\log L_{n}\left(\hat{\theta}_{k}\right)\right)}{n}=A_{2} \quad \text { q.c. }
$$

$e$

$$
\underset{n \rightarrow \infty}{\limsup }\left\|\frac{\sqrt{n}\left(\theta_{r}-\hat{\theta}_{k}\right)}{\sqrt{2 \log \log n}}\right\| \leq c_{4} \quad \text { q.c. }
$$

então

$$
\limsup _{n \rightarrow \infty} \frac{\log L_{n}\left(\hat{\theta}_{k}\right)-\log L_{n}\left(\hat{\theta}_{r}\right)}{\log \log n} \leq 2 c_{4}^{2} \lambda_{1} \quad \text { q.c., }
$$

onde $\lambda_{1}$ é o maior autovalor de $A_{2}$.

(ii) Se existe matriz $A_{2}$ positiva definida tal que, para $\dot{\theta}=s \theta_{r}+(1-s) \hat{\theta}_{k}$ e $s \in[0,1]$,

$$
\lim _{n \rightarrow \infty}-\frac{D_{\theta}^{2}\left(\log L_{n}(\dot{\theta})\right)}{n}=A_{2} \quad \text { q.c. },
$$

e

$$
\limsup _{n \rightarrow \infty}\left\|\frac{D_{\theta}^{1} \log L_{n}\left(\theta_{r}\right)}{\sqrt{2 n \log \log n}}\right\| \leq c_{5} \quad \text { q.c. }
$$

então, para todo $i \in\{1, \ldots, \gamma(k)\}$,

$$
\limsup _{n \rightarrow \infty}\left|\frac{\sqrt{n}\left(\hat{\alpha}_{i}-\alpha_{i}\right)}{\sqrt{2 \log \log n}}\right|<\infty \quad q . c .
$$

$e$

$$
\limsup _{n \rightarrow \infty} \frac{\log L_{n}\left(\hat{\theta}_{k}\right)-\log L_{n}\left(\hat{\theta}_{r}\right)}{\log \log n} \leq \frac{2 c_{5}^{2}}{\lambda_{\gamma(k)}} \quad \text { q.c. }
$$

onde $\lambda_{\gamma(k)}$ é o menor autovalor de $A_{2}$.

(iii) Se para todo $(i, j) \in\{1, \ldots, \gamma(k)\}^{2}$,

$$
\limsup _{n \rightarrow \infty}\left|\frac{\sqrt{n}\left(\hat{\alpha}_{i}-\alpha_{i}\right)}{\sqrt{2 \log \log n}}\right| \leq c_{6} \quad \text { q.c. }
$$


$e$

$$
\lim _{n \rightarrow \infty}\left(\frac{D_{\theta}^{2}\left(\log L_{n}\left(\hat{\theta}_{k}\right)\right)}{n}\right)_{i, j} \leq c_{7} \quad \text { q.c. }
$$

então

$$
\limsup _{n \rightarrow \infty} \frac{\log L_{n}\left(\hat{\theta}_{k}\right)-\log L_{n}\left(\hat{\theta}_{r}\right)}{\log \log n} \leq 2 \gamma(k)^{2} c_{6}^{2} c_{7} \quad \text { q.c. }
$$

Teorema 1.12. Seja $\mathbb{X}$ um processo estocástico a tempo discreto com valores em $\mathbb{R}^{m}, \mathbb{M}$ sua respectiva classe de modelos parcialmente aninhados, $k \geq r, L_{n}\left(\hat{\theta}_{k}\right)$ como na Definição 1.3, $\theta_{r}=\left(\alpha_{1}, \ldots, \alpha_{\gamma(k)}\right) \in \Theta_{k}, \hat{\theta}_{k}=\left(\hat{\alpha}_{1}, \ldots, \hat{\alpha}_{\gamma(k)}\right) \in \Theta_{k}, \hat{\theta}_{k}$ o estimador de máxima verossimilhança de $\theta_{r}$ e valem as Condições de Regularidade (Condição 1.2). Se existe matriz $A_{2}$ positiva definida tal que

$$
\lim _{n \rightarrow \infty}-\frac{D_{\theta}^{2}\left(\log L_{n}\left(\hat{\theta}_{k}\right)\right)}{n}=A_{2} \quad \text { q.c. },
$$

para todo $(i, j) \in\{1, \ldots, \gamma(k)\}^{2}$,

$$
\limsup _{n \rightarrow \infty}\left|\frac{\sqrt{n}\left(\hat{\alpha}_{i}-\alpha_{i}\right)}{\sqrt{\log \log n}}\right|<\infty \quad \text { q.c. }
$$

$e$, para todo $h_{n}$, tal que $\lim _{n \rightarrow \infty} h_{n}=\infty$,

$$
\lim _{n \rightarrow \infty} P\left(\left\|\sqrt{n}\left(\theta_{r}-\hat{\theta}_{k}\right)\right\|>h_{n}\right)=0,
$$

então

$$
\lim _{n \rightarrow \infty} \mathrm{P}\left(\log L_{n}\left(\hat{\theta}_{k}\right)-\log L_{n}\left(\hat{\theta}_{r}\right)>h_{n}\right)=0
$$

para todo $h_{n}$, tal que $\lim _{n \rightarrow \infty} h_{n}=\infty$. 


\section{Cadeias de Markov de espaço de estados gerais}

Para se obter resultados sobre a consistência do estimador EDC é necessário garantir o comportamento assintótico das funções $\log L_{n, k}$, que no fundo se resume em versões aplicadas da Lei Forte dos Grandes Números (LFGN), Lei do Logaritmo Iterado (LLI) e Teorema do Limite Central (TLC).

Para a classe dos processos que possuem dependência finita, o ambiente mais geral é o de cadeias de Markov com espaço de estados gerais. Nesse contexto podem ser inseridos os seguintes.

- Modelo Linear em Espaço de Estados (LSS), que tem como caso particular o processo Autoregressivo (AR).

- Processos Autoregressivos com Médias Móveis (ARMA), que tem o casos particulares o processo de Médias Móveis (MA) e os Autoregressivos (AR).

- A família de processos ARCH, que incluem GARCH (Bollerslev 1986), NGARCH (Engle \& Ng 1993), EGARCH (Nelson 1991) e variações multivariadas como BEKKGARCH (Engle \& Kroner 1995), VEC-GARCH (Bollerslev, Engle \& Wooldridge 1988), CCC-GARCH (Bollerslev 1990), dentre outros.

- Modelos Não-Lineares em Espaço de Estados, que incluem modelos Multilineares em Espaço de Estados.

Todavia, ser inserido no ambiente de cadeias de Markov não é suficiente para a conclusão dos resultados assintóticos. Ainda é necessário o estabelecimento de condições para obter a 
ergodicidade geométrica ou ergodicidade V-uniforme para ser possível o uso dos resultados assintóticos. Mesmo assim, há um grande trabalho desenvolvido para a definição dessas condições em cada caso particular.

Nas últimas décadas, cadeias de Markov teve um grande desenvolvimento, dentre muitos outros, citamos os trabalhos de Kolmogorov (1936), Doeblin (1937, 1940), Foster (1953), Harris (1956), Rosenblatt (1964, 1974), Doob (1966), Feller (1968), Orey (1959, 1971), Cogburn (1972), Tweedie (1974, 1975, 1976), Athreya \& Ney (1978, 1980), Nummelin (1978, 1984) e Niemi \& Nummelin (1982). A parte significativa desse desenvolvimento, acrescida de novos resultados, pode ser encontrada em Meyn \& Tweedie (1993), que é a referência mais importante na área.

Nesse capítulo aplicamos os resultados do capítulo anterior na estimação de ordem de processos Autoregressivos (AR), Autoregressivos de Heteroscedasticidade Condicional $(\mathrm{ARCH})$ e Autoregressivos de Heteroscedasticidade Condicional Generalizado na Modelagem BEKK (BEKK-GARCH). Para processos AR foi possível encontrar a ergodicidade V-uniforme suficiente para a aplicação da LLI, necessária para a conclusão da hipótese H2. Porém, para os outros casos a ergodicidade V-uniforme ainda não foi estabelecida de forma suficiente para concluir H2. Entretanto, as condições para aplicação da LFGN no contexto de cadeias de Markov estão atendidas e são utilizadas. Em razão disso, foi necessário a aplicação da LLI estabelecida para Martingales por Hall \& Heyde (1980).

Na Seção 2.1 são apresentados resumidamente os principais resultados de Meyn\&Tweedie. Nas Seções subsequentes, esses resultados são utilizados para a definição de estimadores de ordem EDC para os processos AR, ARCH e BEKK-GARCH.

\subsection{Definições e principais resultados}

A Definição abaixo expõe os conceitos relacionados à existência e estacionariedade de cadeias de Markov. As Definições 2.2 e 2.3 versam sobre conceitos relacionados a recorrência e ergodicidade, respectivamente.

Definição 2.1. Seja भY um espaço topológico e $\mathscr{B}(\mathscr{Y})$ sua $\sigma$-álgebra de Borel.

(i) $\mathrm{P}: \mathscr{Y} \times \mathscr{B}(\mathscr{Y}) \rightarrow[0,1]$ é núcleo de transição se 
(I) Para todo $A \in \mathscr{B}(\mathscr{Y}), \mathrm{P}(\cdot, A)$ é não-negativa e mensurável em $(\mathscr{Y}, \mathscr{B}(\mathscr{Y}))$;

(II) Para todo $y \in \mathscr{Y}, \mathrm{P}(y, \cdot)$ é medida de probabilidade em $\mathscr{B}(\mathscr{Y})$.

(ii) Um processo estocástico $\mathbb{Y}=\left\{Y_{t}\right\}_{t \in \mathbb{N}}$ é cadeia de Markov (homogênea no tempo) com núcleo de transição $\mathrm{P}(y, A)$ e distribuição inicial $\pi$ se a distribuição de dimensão finita de $\mathbb{Y}$ satisfaz, para todo $n$ e para todo $A_{i} \in \mathscr{B}(\mathscr{Y}), i=1, \cdots, n$,

$$
\mathrm{P}_{\pi}\left(Y_{1} \in A_{1}, \cdots, Y_{n} \in A_{n}\right)=\int_{y_{1} \in A_{1}} \cdots \int_{y_{n-1} \in A_{n-1}} \pi\left(d y_{1}\right) \mathrm{P}\left(y_{1}, d y_{2}\right) \cdots \mathrm{P}\left(y_{n-1}, A_{n}\right) .
$$

(iii) $\mathrm{P}^{n}(y, A)$ é definida indutivamente por

$$
\mathrm{P}^{n}(y, A)=\int_{z \in \mathscr{Y}} \mathrm{P}(y, d z) \mathrm{P}^{n-1}(z, A) \quad y \in \mathscr{Y} \text { e } A \in \mathscr{B}(\mathscr{Y})
$$

e $\mathrm{P}_{y}$ é a medida estendida à $\sigma$-álgebra produto $\vee_{i=1}^{\infty} \mathscr{B}(\mathscr{Y})$ usando o sistema de distribuições

$$
\mathrm{P}_{y}^{1}(A)=\mathrm{P}(x, A)
$$

$e$

$$
\mathrm{P}_{y}^{n}\left(A_{1} \times \cdots \times A_{n}\right)=\int_{A_{1}} \mathrm{P}\left(y, d y_{1}\right) \int_{A_{2}} \mathrm{P}\left(y_{1}, d y_{2}\right) \cdots \mathrm{P}\left(y_{n-1}, A_{n}\right) .
$$

(iv) Uma distribuição $\sigma$-finita $\pi$ em $\mathscr{B}(\mathscr{Y})$ é invariante se para todo $A \in \mathscr{B}(\mathscr{Y})$

$$
\pi(A)=\int_{y} \pi(d y) \mathrm{P}(y, A) .
$$

(v) O tempo de ocupação de A ou número de visitas a A é definido por

$$
\eta_{A}=\sum_{t=1}^{\infty} \mathbb{I}\left(Y_{t} \in A\right)
$$

(vi) O tempo de primeira visita a $A \in \mathscr{B}(\mathscr{Y})$ é definido por

$$
\tau_{A}=\min \left\{t \in \mathbb{N}: Y_{t} \in A\right\}
$$

Definição 2.2. Seja $\mathbb{Y}$ uma cadeia de Markov.

(i) $\mathbb{Y}$ é $\varphi$-irredutivel se existe uma medida $\varphi$ em $\mathscr{B}(\mathscr{Y})$ tal que, se $A \in \mathscr{B}(\mathscr{Y})$ e $\varphi(A)>0$, 
então, $\forall y \in \mathscr{Y}$,

$$
L(y, A)>0
$$

onde $L(y, A):=P_{y}\left(\tau_{A}<\infty\right)$.

(ii) $\mathbb{Y}$ é $\psi$-irredutível se é $\varphi$-irredutível para alguma medida $\varphi$ e $\psi$ é maximal, isto é, $\mathbb{Y}$ é $\varphi^{\prime}$-irredutivel se e somente se $\psi \succ \varphi^{\prime}$.

(iii) Um conjunto $A \in \mathscr{B}(\mathscr{Y})$ é Harris recorrente se, para todo $y \in A$,

$$
Q(y, A):=\mathrm{P}_{y}\left(\eta_{A}=\infty\right)=1 .
$$

(iv) YY é Harris recorrente se é $\psi$-irredutível e todo conjunto em $\{A \in \mathscr{B}(\mathscr{Y}): \psi(A)>0\}$ é Harris recorrente.

(v) $\mathbb{Y}, \psi$-irredutível, é positiva se admite distribuição invariante.

(vi) Y é Harris positiva se é Harris recorrente e positiva.

Definição 2.3. Para $\mathbb{Y}$ uma cadeia de Markov.

(i) Y é ergódica se, $\forall y \in \mathscr{Y}$,

$$
\lim _{n \rightarrow \infty} \sup _{A \in \mathscr{B}(\mathscr{Y})}\left|\mathrm{P}^{n}(y, A)-\pi(A)\right|=0 .
$$

(ii) Para $V: E \rightarrow[1, \infty)$ e v uma medida em $\mathscr{B}(\mathscr{Y})$,

$$
\|v\|_{V}=\sup _{g:|g| \leq V}\left\{\int_{y \in \mathscr{Y}} g(y) v(d y)\right\} .
$$

(iii) Y é V-uniformemente ergódica se

$$
\lim _{n \rightarrow \infty} \sup _{y \in \mathbb{Y}} \frac{\left\|\mathrm{P}^{n}(y, \cdot)-\pi\right\|_{V}}{V(y)}=0 .
$$

No que segue, são apresentadas as chamadas condições do tipo "drift", que têm como objetivo concluir a ergodicidade geométrica ou ergodicidade V-uniforme baseado no comportamento de uma única transição do processo. 
Definição 2.4. Um conjunto $B \in \mathscr{B}(\mathscr{Y})$ é chamado "pequeno" se existe um $m>0$ e uma medida não-trivial $v_{m}$ em $\mathscr{B}(\mathscr{Y})$, tais que, para todo $y \in B$ e $A \in \mathscr{B}(\mathscr{Y})$,

$$
\mathrm{P}^{m}(y, A) \geq v_{m}(A)
$$

Condição 2.1. Para $f: \mathscr{Y} \rightarrow[1, \infty), B \in \mathscr{B}(\mathscr{Y})$ conjunto pequeno, $b<\infty$ e $V: \mathscr{Y} \rightarrow[0, \infty)$,

$$
E\left[V\left(Y_{t+1}\right) \mid Y_{t}\right]-V\left(Y_{t}\right) \leq-f\left(Y_{t}\right)+b \mathbb{I}_{B}\left(Y_{t}\right)
$$

Condição 2.2 (Foster-Lyapunov). Existe $V: \mathscr{Y} \rightarrow[1, \infty), B \in \mathscr{B}(\mathscr{Y})$ conjunto pequeno, $\beta>0$ e $b_{1}<\infty$, tais que

$$
E\left[V\left(Y_{t+1}\right) \mid Y_{t}\right]-V\left(Y_{t}\right) \leq-\beta V\left(Y_{t}\right)+b_{1} \mathbb{I}_{B}\left(Y_{t}\right)
$$

Observa-se que a Condição 2.2 é a Condição 2.1 no caso particular $f=\beta V$. O Teorema 2.5 é uma adaptação dos Teoremas 17.0.1, 17.3.6 e 17.5.3 de Meyn \& Tweedie e é peça fundamental no estabelecimento do comportamento assintótico das funções $\log L_{n, k}$.

Teorema 2.5 (Meyn \& Tweedie (1993)). Seja $\mathbb{Y}=\left\{Y_{t}\right\}_{t \in \mathbb{N}}$ uma cadeia de Markov a tempo discreto com espaço de estados $\mathscr{Y}$, Harris recorrente com distribuição invariante $\pi, g$ : $\mathscr{Y} \rightarrow \mathbb{R} e S_{n}(g)=\sum_{t=1}^{n} g\left(Y_{t}\right)$.

(i) $\mathrm{Se} E_{\pi}(|g|)<\infty$, então

$$
\lim _{n \rightarrow \infty} \frac{S_{n}(g)}{n}=E_{\pi}(g)
$$

(ii) Se $\mathbb{Y}$ é V-uniformemente ergódica, $g^{2} \leq V$ e $\int g d \pi=0$, então $\phi_{g}^{2}:=E_{\pi}\left[g^{2}\left(X_{1}\right)\right]+$ $2 \sum_{k=2}^{\infty} E_{\pi}\left[g\left(X_{1}\right) g\left(X_{k}\right)\right]$ está bem definido.

(iii) Se YY é ergódica, e existe uma função $f: \mathscr{Y} \rightarrow[1, \infty)$, um conjunto pequeno $C, b_{0}<$ $\infty$ satisfazendo (2.1), $\pi\left(V^{2}\right)<\infty,|g| \leq f e \int g d \pi=0$, então $\phi_{g}^{2}:=E\left[g^{2}\left(Y_{1}\right)\right]+$ $2 \sum_{k=2}^{\infty} E\left[g\left(Y_{1}\right) g\left(Y_{k}\right)\right]$ está bem definido.

(iv) Nas hipóteses de (ii) ou nas hipóteses de (iii), se $\phi_{g}^{2}>0$, então

$$
\lim _{n \rightarrow \infty} \frac{S_{n}(g)}{\sqrt{n \phi_{g}^{2}}} \stackrel{d}{\rightarrow} \mathscr{N}(0,1),
$$




$$
\begin{aligned}
& \limsup _{n \rightarrow \infty} \frac{S_{n}(g)}{\sqrt{2 n \phi_{g}^{2} \log \log (n)}}=1 \quad \text { q.c. } e \\
& \liminf _{n \rightarrow \infty} \frac{S_{n}(g)}{\sqrt{2 n \phi_{g}^{2} \log \log (n)}}=-1 \quad \text { q.c. }
\end{aligned}
$$

A Lei do Logaritmo Iterado para Martingales, transcrita abaixo, pode ser encontrada em Hall \& Heyde (1980), Teoremas 4.7 e 4.8.

Teorema 2.6 (Hall \& Heyde (1980)). Seja $\left\{S_{n}, \mathscr{F}_{t-1}\right\}$ uma martingale, $S_{n}=\sum_{t=1}^{n} U_{t}, E\left(S_{n}\right)=$ 0, $E\left(S_{n}^{2}\right)<\infty,\left\{Z_{t}\right\}_{t \in \mathbb{N}} e\left\{W_{t}\right\}_{t \in \mathbb{N}}$ sequências de variáveis aleatórias não negativas tais que $Z_{t}$ e $W_{t}$ são $\mathscr{F}_{t-1}$ mensuráveis. Se

$$
\begin{gathered}
\lim _{n \rightarrow \infty} \frac{\sum_{t=1}^{n} U_{t} \mathbb{I}\left(\left|U_{t}\right|>Z_{t}\right)-E\left[U_{t} \mathbb{I}\left(\left|U_{t}\right|>Z_{t}\right) \mid \mathscr{F}_{t-1}\right]}{\sqrt{2 W_{n}^{2} \log \log W_{n}^{2}}}=0 \quad \text { q.c. }, \\
\lim _{n \rightarrow \infty} \frac{\sum_{t=1}^{n} E\left[U_{t}^{2} \mathbb{I}\left(\left|U_{t}\right| \leq Z_{t}\right) \mid \mathscr{F}_{t-1}\right]-E\left[U_{t} \mathbb{I}\left(\left|U_{t}\right| \leq Z_{t}\right) \mid \mathscr{F}_{t-1}\right]^{2}}{W_{n}^{2}}=1 \quad \text { q.c. } \\
\lim _{n \rightarrow \infty} \sum_{t=1}^{n} \frac{E\left[U_{t}^{4} \mathbb{I}\left(\left|U_{t}\right| \leq Z_{t}\right) \mid \mathscr{F}_{t-1}\right]}{W_{t}^{4}}<\infty \quad \text { q.c. } \\
\lim _{n \rightarrow \infty} \frac{W_{n}}{W_{n+1}}=1 \quad \text { q.c.e } \lim _{n \rightarrow \infty} W_{n}=\infty \quad \text { q.c. }
\end{gathered}
$$

Então

$$
\limsup _{n \rightarrow \infty} \frac{S_{n}}{\sqrt{2 W_{n}^{2} \log \log W_{n}^{2}}}=1 \quad \text { q.c. }
$$

$e$

$$
\liminf _{n \rightarrow \infty} \frac{S_{n}}{\sqrt{2 W_{n}^{2} \log \log W_{n}^{2}}}=-1 \quad \text { q.c. }
$$

\subsection{Modelo Autoregressivo (AR)}

O processo Autoregressivo (AR) foi proposto inicialmente por Yule (1921) para a modelagem de séries temporais e vem sendo utilizado em diversas áreas, dentre outras citamos econometria (Maddala \& Lahiri 2009), engenharia (Schneider 1988) e genética (Carvalheira, Blake, Pollak, Quaas \& Duran-Castro 1998). 
O problema de estimação de ordem em modelos AR inicialmente foi abordado usando testes de hipóteses por Quenouille (1947), Whittle $(1951,1954)$ e Bartlett \& Rajalakshman (1953). Akaike (1969) propôs o método de minimização do erro final de predição (FPE - Final Prediction Error) para a estimação da ordem em modelos AR, que foi o precursor do método AIC, proposto pelo mesmo autor em 1974. Após isso, foram desenvolvidos outros métodos de estimação, que se destacam o BIC (Akaike 1979) e HQC (Hannan \& Quinn 1979). Vale ressaltar que o problema de estimação de ordem em modelos AR motivaram a criação do AIC e influenciou o desenvolvimento de estimadores de ordem em outros processos, como exemplo em cadeias de Markov. Para uma visão mais detalhada sobre o desenvolvimento das técnicas de estimação de ordem em processo ARMA (que tem o AR como caso particular), veja Choi (1992).

No que segue, o ferramental desenvolvido nesse trabalho é aplicado para a estimação da ordem em modelos AR e um novo estimador de ordem é proposto. Também são apresentadas simulações numéricas que indicam, no geral, uma melhor performance do estimador proposto quando comparado com as alternativas AIC, BIC e HQC.

\subsubsection{Definições}

Abaixo o processo AR é definido para inovações Gaussianas e em seguida é apresentada a Condição 2.3, que é requisito clássico para a obtenção de resultados assintóticos.

Definição 2.7. Uma sequência de variáveis aleatórias $\mathbb{X}=\left\{X_{t}\right\}_{t \in \mathbb{Z}}$ com valores em $\mathbb{R}$ é um processo Autoregressivo de ordem $r \in \mathbb{N}$, denotado por $A R(r)$, se satisfaz as condições abaixo.

(i) Para todo $t \in \mathbb{Z}$,

$$
X_{t}=h_{t}+\varepsilon_{t}
$$

(ii) $h_{t}=\alpha_{1} X_{t-1}+\cdots+\alpha_{r} X_{t-r}, \theta_{r}=\left(\alpha_{1}, \ldots, \alpha_{r}\right) \in \mathbb{R}^{r}$ e $\alpha_{r} \neq 0$.

(iii) $\left\{\varepsilon_{t}\right\}_{t \in \mathbb{Z}}$ é uma sequência de variáveis aleatórias i.i.d. $\mathscr{N}\left(0, \sigma^{2}\right)$. 
Condição 2.3 (Ergodicidade). Para $\mathbb{X} u m A R(r)$, e $\theta_{r}=\left(\alpha_{1}, \ldots, \alpha_{r}\right) \in \mathbb{R}^{r}$ seus respectivos parâmetros, assumimos que

$$
1-\sum_{i=1}^{r} \alpha_{i} z^{i} \neq 0 \quad \forall z \in \mathbb{C},|z| \leq 1
$$

\section{Principais propriedades do processo autoregressivo}

Algumas propriedade básicas para processos AR, que são utilizadas no desenvolvimento dessa Seção, são apresentadas abaixo.

(a) $E\left(X_{i} \mid \mathscr{F}_{i-1}\right)=h_{i}$ q.c.

(b) Um $\mathrm{AR}(\mathrm{r}), \mathbb{X}$, pode ser imerso em uma sequência de modelos aninhados $\mathbb{M}=\bigcup_{k=0}^{\infty} M_{k}$, tomando $\Theta_{k}=\mathbb{R}^{k}$ e, para $\theta_{k}=\left(\alpha_{1}, \cdots, \alpha_{k}\right) \in \Theta_{k}$,

$$
f\left(x_{1}^{n}, \theta_{k}\right)=C\left(x_{1}^{k}\right) \prod_{t=1+k}^{n} \frac{1}{\sqrt{2 \pi \sigma^{2}}} e^{-\frac{\left(x_{t}-\left(\alpha_{1} x_{t-1}+\cdots+\alpha_{k} x_{t-k}\right)\right)^{2}}{2 \sigma^{2}}}
$$

(c) Usando que $\mathbb{X}$ é estacionário e (2.3), temos que

$$
0<|m(k)|<\infty
$$

onde $m(k)=E\left(X_{t} X_{t+k}\right)$ e $t, k \in \mathbb{Z}$ (Anderson (1994), página 173).

Funções $\log L_{n, k}$ e suas derivadas

A log-verossimilhança de $m_{k}=f\left(x_{1}^{n}, \theta_{k}\right) \in M_{k}$ é dada por

$$
\begin{aligned}
\log \left(\prod_{t=1+k}^{n} f\left(X_{t} \mid X_{t-k-1}^{t-1}\right)\right)+C_{1}\left(X_{1}^{k}\right) & =\sum_{t=1+k}^{n} \log \left(\frac{1}{\sqrt{2 \pi \sigma^{2}}} e^{-\frac{\left(X_{t}-h_{t}\right)^{2}}{2 \sigma^{2}}}\right)+C_{1}\left(X_{1}^{k}\right) \\
& =\sum_{t=1+k}^{n}-\frac{\left(X_{t}-h_{t}\right)^{2}}{2 \sigma^{2}}-(n-k) \log \sqrt{2 \pi \sigma^{2}}+C_{1}\left(X_{1}^{k}\right) .
\end{aligned}
$$

Definimos abaixo o logaritmo de $L_{n, k}$, que satisfaz a Definição 1.3.

$$
\log L_{n, k}(\theta)=\sum_{t=1+k}^{n}-\frac{\left(X_{t}-h_{t}\right)^{2}}{2}
$$


Dessa forma, para $i, j, l \in\{1, \cdots, k\}$,

$$
\begin{gathered}
\frac{\partial \log L_{n, k}(\theta)}{\partial \alpha_{i}}=\sum_{t=1+k}^{n}\left(X_{t}-h_{t}\right) X_{t-i} \\
\frac{\partial^{2} \log L_{n, k}(\theta)}{\partial \alpha_{i} \alpha_{j}}=\sum_{t=1+k}^{n}-X_{t-i} X_{t-j} e \\
\frac{\partial^{3} \log L_{n, k}(\theta)}{\partial \alpha_{i} \alpha_{j} \alpha_{l}}=0
\end{gathered}
$$

Alguns resultados disponíveis na literatura são estabelecidos para modelos Lineares em Espaço de Estados (LSS), que possui como caso particular os modelos AR. Nesse sentido, definimos abaixo modelos LSS e apresentamos condições para a existência de ergodicidade V-uniforme. Esses resultados podem ser encontrados de forma mais geral em Meyn \& Tweedie (1993).

Definição 2.8. Uma sequência de vetores aleatórios $\mathbb{Y}=\left\{Y_{t}\right\}_{t \in \mathbb{Z}}$ com valores em $\mathbb{R}^{r}$ é um modelo Linear em Espaço de Estados (LSS) se

(i) Existe matriz $(r \times r)$ F e matriz $(r \times p) G$ tal que para todo $t \in \mathbb{N}$, $Y_{t}$ e $W_{t}$ com respectivos valores em $\mathbb{R}^{r}$ e $\mathbb{R}^{p}$ satisfazem, para todo $t \in \mathbb{N}$,

$$
Y_{t}=F Y_{t-1}+G W_{t}
$$

e $Y_{0}$ é arbitrário.

(ii) Os vetores aleatórios $\left\{W_{t}\right\}_{t \in \mathbb{N}}$ são i.i.d., são independentes de $Y_{0}$ e possuem distribuição com média e variância finitas.

Condição 2.4. Se $\mathbb{Y}$ é um modelo LSS, então

(i) W possui distribuição Gaussiana em $\mathbb{R}^{p}$ com média zero e variância 1 . Isto é, $W \sim$ $\mathscr{N}(0, I)$, I a matriz identidade $(p \times p)$.

(ii) Se $\lambda \in \mathbb{C}$ é autovalor de $F$, então $|\lambda|<1$.

Se $\mathbb{X}$ é um AR(r), podemos representá-lo como um modelo Linear de Espaço de Estados 
$\mathbb{Y}$ tomando $p=1, Y_{t}=\left(X_{t}, \ldots, X_{t-r+1}\right)$ com valores em $\mathbb{R}^{r}, W_{t}=\varepsilon_{t}, \varepsilon_{t} \sim \mathscr{N}(0,1)$,

$$
F=\left[\begin{array}{cccc}
\alpha_{1} & \alpha_{2} & \ldots & \alpha_{r} \\
1 & 0 & \ldots & 0 \\
0 & \ddots & & \vdots \\
0 & \ldots & 1 & 0
\end{array}\right] \quad \text { e } G=\left[\begin{array}{c}
\sigma \\
0 \\
\vdots \\
0
\end{array}\right]
$$

Usando isso, podemos usar o ferramental desenvolvido para cadeias de Markov, no que se refere a estabilidade assintótica. Em outras palavras, temos o seguinte resultado.

Lema 2.9 (Meyn \& Tweedie (1993) e Anderson (1994)). Se um AR(r) X satisfaz (2.3), então $\mathbb{Y}$, o modelo Linear de Espaço de Estados correspondente conforme definido em (2.11), satisfaz a Condição 2.4, é Harris positivo e V-uniformemente ergódico para $V(x)=|x|^{2}+1$. Além disso, Y satisfaz a Condição 2.2 para $\beta \in(0, \infty), b_{1} \in(-\infty, \infty)$ e B conjunto pequeno e compacto.

Demonstração. Temos que $\mathbb{Y}$ satisfaz a Condição 2.4 (Anderson (1994) página 180). Usando a Proposição 4.4.3 de Meyn \& Tweedie (1993) temos que as condições dos Teoremas 12.5.1 e 17.6.2 de Meyn \& Tweedie (1993) são satisfeitas e portanto $\mathbb{Y}$ é Harris positiva e é V-uniformemente ergódico para $V(x)=|x|^{2}+1$. A demonstração do Teorema 17.6.2 de Meyn\&Tweedie mostra que $\mathbb{Y}$ satisfaz a Condição 2.2.

\subsubsection{Consistência do estimador de ordem de dependência}

Abaixo é definido estimador de ordem EDC para o caso particular de processos AR. Em seguida estão uma série de resultados com o objetivo de demonstrar as hipóteses H1-H4 do Teorema 1.5 e concluir os casos de convergência do estimador proposto, que estão sumarizados no Teorema 2.18. Como mencionado anteriormente, a técnica de utilizar o comportamento assintótico das primeiras derivadas de $\log L_{n, k}$ para concluir o comportamento assintótico de $\log L_{n, k}$ foi utilizada por Nishii (1988) em caso particular de estimação de dimensão de modelos i.i.d. e considerando $\log L_{n, k}$ como a $\log$-verossimilhança. Ressaltase também que, de forma semelhante, Basawa \& Heyde (1976) utiliza o comportamento assintótico das primeiras derivadas da log-verossimilhança para concluir o comportamento assintótico do estimador de máxima verossimilhança. A essência dessa técnica é a utilização 
da expansão em séries de Taylor em determinados pontos, para isso são exigidas as condições de regularidade das derivadas de $\log L_{n, k}$.

Definição 2.10. Para $\mathbb{X} u m A R(r)$ e $K \geq r$, definimos o estimador EDC de r por

$$
\hat{r}_{e d c}=\underset{k \in\{0, \ldots, K\}}{\operatorname{argmin}}\left\{-\log L_{n}\left(\hat{\theta}_{k}\right)+k c_{n}\right\}
$$

para $\log L_{n}\left(\hat{\theta}_{k}\right)$ como definida em (2.6) $e\left\{c_{n}\right\}_{n \in \mathbb{N}}$ uma sequência de números positivos.

Lema 2.11. Seja $\mathbb{X}=\left\{X_{t}\right\}_{t \in \mathbb{Z}} u m A R(r), k \geq r, \log L_{n}\left(\hat{\theta}_{k}\right)$ como definida (2.6), $\hat{\theta}_{k} \in \Theta_{k} \subseteq \mathbb{R}^{k}$ o estimador de máxima verossimilhança, então

(i) Para $\dot{\theta}=s \theta_{r}+(1-s) \hat{\theta}_{k}, s \in[0,1]$,

$$
\lim _{n \rightarrow \infty}-\frac{D_{\theta}^{2} \log L_{n}(\dot{\theta})}{n}=A_{2} \quad \text { q.c. }
$$

onde

$$
A_{2}=\left[\begin{array}{ccc}
m(0) & \ldots & m(k) \\
\vdots & \ddots & \vdots \\
m(k) & \ldots & m(0)
\end{array}\right]
$$

e $m(k)=E\left(X_{t} X_{t+k}\right)$

(ii) $\mathrm{A}_{2}$ é positiva definida.

Demonstração. (i) Usando a desigualdade de Hölder

$$
E\left(\left|X_{t-i} X_{t-j}\right|\right) \leq E\left(X_{t-i}^{2}\right)^{\frac{1}{2}} E\left(X_{t-j}^{2}\right)^{\frac{1}{2}}<\infty
$$

Pelo Teorema 2.5 e expressão (2.8),

$$
\lim _{n \rightarrow \infty} \frac{\frac{\partial^{2} \log L_{n}(\dot{\theta})}{\partial \alpha_{i} \alpha_{j}}}{n}=-E\left(X_{t-i} X_{t-j}\right)=-m(i-j) .
$$

(ii) Demonstração pode ser encontrada em Anderson (1994), Lema 5.5.4, página 196. 
Lema 2.12. Seja $\mathbb{X}=\left\{X_{t}\right\}_{t \in \mathbb{Z}} u m A R(r), k \geq r, \log L_{n, k}(\theta)$ como definida (2.6), $i \in\{1, \cdots, k\}$, então

$$
\begin{gathered}
\limsup _{n \rightarrow \infty} \frac{\frac{\partial \log L_{n, k}\left(\theta_{r}\right)}{\partial \alpha_{i}}}{\sqrt{2 n \log \log n}}=\sqrt{\sigma^{2} E\left(X_{1}^{2}\right)} \quad \text { q.c., } \\
\liminf _{n \rightarrow \infty} \frac{\frac{\partial \log L_{n, k}\left(\theta_{r}\right)}{\partial \alpha_{i}}}{\sqrt{2 n \log \log n}}=-\sqrt{\sigma^{2} E\left(X_{1}^{2}\right)} \quad \text { q.c. } e \\
\frac{\frac{\partial \log L_{n, k}\left(\theta_{r}\right)}{\partial \alpha_{i}}}{\sqrt{n}} \underset{D}{M} \mathscr{N}\left(0, \sigma^{2} E\left(X_{1}^{2}\right)\right) \quad \text { q.c. }
\end{gathered}
$$

Demonstração. Assumindo $\mathscr{F}_{t}$ a $\sigma$-álgebra gerada por $\left\{X_{l}\right\}_{l \leq t}$ e considerando o Teorema 2.5, $\left\{Y_{t}\right\}_{t \in \mathbb{Z}}$ o modelo LSS associado, $g\left(Y_{t}\right)=\left(X_{t}-h_{t}\right) X_{t-i}, E(g)=E\left(X_{t-i} E\left(\varepsilon_{t} \mid \mathscr{F}_{t}\right)\right)=0 \mathrm{e}$

$$
\phi_{g}^{2}=E\left[\left(\left(X_{1}-h_{1}\right) X_{1-i}\right)^{2}\right]+2 \sum_{t=k+2}^{\infty} E\left[\left(\left(X_{1}-h_{1}\right) X_{1-i}\right)\left(\left(X_{t}-h_{t}\right) X_{t-i}\right)\right] .
$$

Para a primeira parcela

$$
\begin{aligned}
E\left[\left(\left(X_{1}-h_{1}\right) X_{1-i}\right)^{2}\right] & =E\left[X_{1-i}^{2} E\left(\varepsilon_{1}^{2} \mid \mathscr{F}_{0}\right)\right] \\
& =\sigma^{2} E\left(X_{1-i}^{2}\right) .
\end{aligned}
$$

Usando (2.5), concluímos

$$
0<E\left[\left(\left(X_{1}-h_{1}\right) X_{1-i}\right)^{2}\right]<\infty
$$

Para a segunda parcela

$$
\begin{aligned}
E\left[\left(\left(X_{1}-h_{1}\right) X_{1-i}\right)\left(\left(X_{t}-h_{t}\right) X_{t-i}\right)\right] & =E\left[\left(X_{1}-h_{1}\right) X_{1-i} X_{t-i} E\left(\left(X_{t}-h_{t}\right) \mid \mathscr{F}_{t-1}\right)\right] \\
& =0 .
\end{aligned}
$$

Então

$$
\phi_{g}^{2}=\sigma^{2} E\left(X_{1-i}^{2}\right)
$$


Também temos que

$$
\begin{aligned}
\left|g\left(Y_{t}\right)\right| & =\left|\left(X_{t}-h_{t}\right) X_{t-i}\right| \\
& \leq(r+1) \max \left\{1,\left|\alpha_{1}\right|, \cdots,\left|\alpha_{r}\right|\right\} \max _{l=t-r, \ldots, t}\left|X_{l}\right|^{2} \\
& \leq d_{1}\left|Y_{t}\right|^{2} \\
& \leq d_{1} V\left(Y_{t}\right) .
\end{aligned}
$$

Na penúltima desigualdade usamos que todas as normas são equivalentes em espaços de dimensão finita. De (2.14) e (2.2) concluímos (2.1) para $f=\max \{1,|g|\}, b_{0}=\left(b_{1}+\beta / d_{1}\right)$ e $C=B \cup\left\{x \in R^{r} ;|V(x)| \leq 1 / d_{1}\right\}$. Também $\pi\left(V^{2}\left(Y_{t}\right)\right) \leq d_{2} \pi\left(\max _{i=t-r . . r}\left|Y_{t}\right|^{4}\right)<\infty$ (Anderson 1994).

Observando que se (2.1) vale para $\frac{\beta}{d_{1}} g$ então vale para $g$, podemos usar o Teorema 2.5 e concluir

$$
\limsup _{n \rightarrow \infty} \frac{\frac{\partial \log L_{n, k}\left(\theta_{r}\right)}{\partial \alpha_{i}}}{\sqrt{2 n \sigma^{2} E\left(X_{1}^{2}\right) \log \log n}}=1 \text { q.c. }
$$

Então,

$$
\limsup _{n \rightarrow \infty} \frac{\frac{\partial \log L_{n, k}\left(\theta_{r}\right)}{\partial \alpha_{i}}}{\sqrt{2 n \log \log n}}=\sqrt{\sigma^{2} E\left(X_{1}^{2}\right)} \quad \text { q.c. }
$$

Da mesma forma concluímos (2.12) e (2.13).

Corolário 2.13. Seja $\left\{X_{t}\right\}_{t \in \mathbb{Z}}$ um AR(r) satisfazendo (2.3), $k \geq r, \hat{\theta}_{k}=\left(\hat{\alpha}_{1}, \cdots, \hat{\alpha}_{k}\right)$ o estimador de máxima verossimilhança de $\theta_{r}=\left(\alpha_{1}, \cdots, \alpha_{r}, 0, \cdots, 0\right) \in \mathbb{R}^{k}$ e $h_{n}$, tal que $\lim _{n \rightarrow \infty} h_{n}=\infty$, então

$$
\lim _{n \rightarrow \infty} \mathrm{P}\left(\left\|\sqrt{n}\left(\theta_{r}-\hat{\theta}_{k}\right)\right\|>h_{n}\right)=0 .
$$


Demonstração. Usando o Lema 2.12 e que para $n$ grande $A_{n}$ é positiva definida temos

$$
\begin{aligned}
\lim _{n \rightarrow \infty} \mathrm{P}\left(\left\|\sqrt{n}\left(\theta_{r}-\hat{\theta}_{k}\right)\right\|>h_{n}\right) & =\lim _{n \rightarrow \infty} \mathrm{P}\left(\left\|\frac{D_{\theta}^{1} \log L_{n, k}\left(\theta_{r}\right) A_{n}^{-1}}{\sqrt{n}}\right\|>h_{n}\right) \\
& \leq \lim _{n \rightarrow \infty} \mathrm{P}\left(\frac{1}{\lambda_{k}}\left\|\frac{D_{\theta}^{1} \log L_{n, k}\left(\theta_{r}\right)}{\sqrt{n}}\right\|>h_{n}\right) \\
& =\lim _{n \rightarrow \infty} \mathrm{P}\left(\sum_{i=1}^{k}\left|\frac{\frac{\partial \log L_{n, k}\left(\theta_{r}\right)}{\partial \alpha_{i}}}{\sqrt{n}}\right|^{2}>\lambda_{k}^{2} h_{n}^{2}\right) \\
& =0 .
\end{aligned}
$$

Onde $1 / \lambda_{k}$ é o maior autovalor de $A_{n}^{-1}$.

Corolário 2.14. Para um $A R(r)$, se $k>r$, então

$$
\limsup _{n \rightarrow \infty} \frac{\log L_{n}\left(\hat{\theta}_{k}\right)-\log L_{n}\left(\hat{\theta}_{r}\right)}{\log \log n} \leq \frac{2 k \sigma^{2} E\left(X^{2}\right)}{\lambda_{k}} \text { q.c. }
$$

onde $\lambda_{k}$ é o menor autovalor de $A_{2}$.

Demonstração. A partir do Lema 2.12 temos que

$$
\underset{n \rightarrow \infty}{\limsup }\left\|\frac{D_{\theta}^{1} \log L_{n, k}\left(\theta_{r}\right)}{\sqrt{2 n \log \log n}}\right\|^{2} \leq k \sigma^{2} E\left(X_{1}^{2}\right) .
$$

Aplicando o Teorema 1.6, item (ii), e usando os Lemas 2.11 e 2.12 temos o desejado.

Usando outra técnica, podemos obter o Teorema abaixo, que é útil se for possível determinar o limitante superior em (1.16), ao aplicar o Lema 2.12, de forma a não depender de $k$ ou $r$. Se for possível, podemos encontrar o $c_{n}=O(\log \log n)$ preciso que gera um estimador $\hat{r}_{\text {edc }}$ fortemente consistente.

Teorema 2.15. Se $\mathbb{X}$ é um AR(r) satisfazendo a Condição 2.3 e $k>r$, então

$$
\limsup _{n \rightarrow \infty} \frac{\log L_{n, k}\left(\hat{\theta}_{k}\right)-\log L_{n, r}\left(\hat{\theta}_{r}\right)}{\log \log n} \leq(k-r) c_{2} \quad \text { q.c. }
$$

Demonstração. Tomando a série de Taylor para $\log L_{n, k}\left(\hat{\theta}_{r}\right)$ em $\hat{\theta}_{k}$ e usando (2.9), temos $\log L_{n, k}\left(\hat{\boldsymbol{\theta}}_{r}\right)=\log L_{n, k}\left(\hat{\boldsymbol{\theta}}_{k}\right)+\left(\hat{\boldsymbol{\theta}}_{r}-\hat{\boldsymbol{\theta}}_{k}\right) D_{\theta}^{1}\left(\log L_{n, k}\left(\hat{\boldsymbol{\theta}}_{k}\right)\right)+\frac{1}{2}\left(\hat{\boldsymbol{\theta}}_{r}-\hat{\boldsymbol{\theta}}_{k}\right) D_{\theta}^{2}\left(\log L_{n, k}\left(\hat{\boldsymbol{\theta}}_{k}\right)\right)\left(\hat{\boldsymbol{\theta}}_{r}-\hat{\boldsymbol{\theta}}_{k}\right)^{T}$ 
Como $\log L_{n, k}$ é maximizada por $\hat{\theta}_{k}$, temos que $D_{\theta}^{1}\left(\log L_{n, k}\left(\hat{\theta}_{k}\right)\right)=0$ e

$$
\begin{aligned}
\limsup _{n \rightarrow \infty} \frac{\log L_{n, k}\left(\hat{\theta}_{k}\right)-\log L_{n, k}\left(\hat{\theta}_{r}\right)}{\log \log n} & =\limsup _{n \rightarrow \infty} \frac{-\left(\hat{\theta}_{r}-\hat{\theta}_{k}\right) D_{\theta}^{2}\left(\log L_{n, k}\left(\hat{\theta}_{k}\right)\right)\left(\hat{\theta}_{r}-\hat{\theta}_{k}\right)^{T}}{2 \log \log n} \\
& =\limsup _{n \rightarrow \infty} \frac{\sqrt{n}\left(\hat{\theta}_{r}-\hat{\theta}_{k}\right)}{\sqrt{2 \log \log n}} \frac{-D_{\theta}^{2}\left(\log L_{n, k}\left(\hat{\theta}_{k}\right)\right)}{n} \frac{\left(\sqrt{n}\left(\hat{\theta}_{r}-\hat{\theta}_{k}\right)\right)^{T}}{\sqrt{2 \log \log n}}
\end{aligned}
$$

Usando Shibata (1976), temos que (2.15) pode ser definido como a norma

$$
\|x\|_{1}^{2}:=x \frac{-D_{\theta}^{2}\left(\log L_{n, k}\left(\hat{\theta}_{k}\right)\right)}{n} x^{T}
$$

que satisfaz

$$
\left\|\hat{\theta}_{k}-\hat{\theta}_{r}\right\|_{1}^{2}=\hat{\sigma}_{r}^{2}-\hat{\sigma}_{k}^{2}
$$

onde $\hat{\sigma}_{l}^{2}$ é o estimador de $\sigma^{2}$ se $l \geq r$. Por Hannan \& Quinn (1979),

$$
\hat{\sigma}_{k}^{2}=\hat{\sigma}_{r}^{2} \prod_{i=r+1}^{k}\left(1-\hat{\alpha}_{i}^{2}(i)\right)
$$

onde $\hat{\alpha}_{i}(l)$ é o estimador de máxima verossimilhança de $\alpha_{i}$, considerando $l$ a ordem verdadeira. Usando (2.16) e (2.17)

$$
\begin{aligned}
\limsup _{n \rightarrow \infty} \frac{\log L_{n, k}\left(\hat{\theta}_{k}\right)-\log L_{n, k}\left(\hat{\theta}_{r}\right)}{\log \log n} & =\limsup _{n \rightarrow \infty} \frac{n\left\|\hat{\theta}_{r}-\hat{\theta}_{k}\right\|_{1}^{2}}{2 \log \log n} \\
& =\limsup _{n \rightarrow \infty} \frac{n\left[\hat{\sigma}_{r}^{2}-\hat{\sigma}_{r}^{2} \prod_{i=r+1}^{k}\left(1-\hat{\alpha}_{i}^{2}(i)\right)\right]}{2 \log \log n} \\
& =\limsup _{n \rightarrow \infty} \frac{n \hat{\sigma}_{r}^{2}\left[1-1+\sum_{i=r+1}^{k} \hat{\alpha}_{i}^{2}(i)+o(\log \log n / n)\right]}{2 \log \log n} \\
& \leq \sigma^{2} \sum_{i=r+1}^{k} \limsup _{n \rightarrow \infty}\left(\frac{\sqrt{n}\left(\hat{\alpha}_{i}(i)-0\right)}{\sqrt{2 \log \log n})^{2}}\right. \\
\leq & c_{2}(k-r) .
\end{aligned}
$$

O resultado segue de (1.4). Usamos também que $\hat{\sigma}_{r}^{2} \underset{\text { q.c. }}{\rightarrow} \sigma^{2}$ e a inequação (1.16), quando se aplica o Lema 2.12. O argumento utilizado tem um valor teórico interessante. Entretanto, 
basta verificar diretamente (2.6) e observar que

$$
\log L_{n, k}\left(\hat{\theta}_{k}\right)-\log L_{n, r}\left(\hat{\theta}_{r}\right)=n\left(\hat{\sigma}_{r}^{2}-\hat{\sigma}_{k}^{2}\right)
$$

e continuar as contas a partir de (2.18).

Teorema 2.16. Se $\mathbb{X}$ é um AR(r) satisfazendo a Condição $2.3, k>r$ e $h_{3} \in(0, \infty)$, então

$$
\lim _{n \rightarrow \infty} \mathrm{P}\left(\log L_{n}\left(\hat{\theta}_{k}\right)-\log L_{n}\left(\hat{\theta}_{r}\right)>h_{3}\right)>0
$$

Demonstração. Usando (2.6) e Hannan \& Quinn (1979), temos

$$
\begin{aligned}
\lim _{n \rightarrow \infty} \mathrm{P}\left(\log L_{n, k}\left(\hat{\theta}_{k}\right)-\log L_{n, r}\left(\hat{\theta}_{r}\right)>h_{3}\right) & =\lim _{n \rightarrow \infty} \mathrm{P}\left(n\left(\hat{\sigma}_{r}^{2}-\hat{\sigma}_{k}^{2}\right)>h_{3}\right) \\
& =\lim _{n \rightarrow \infty} \mathrm{P}\left(n\left[\hat{\sigma}_{r}^{2}-\hat{\sigma}_{r}^{2} \prod_{i=r+1}^{k}\left(1-\hat{\alpha}_{i}^{2}(i)\right)\right]>h_{3}\right) \\
& =\lim _{n \rightarrow \infty} \mathrm{P}\left(n \hat{\sigma}_{r}^{2}\left[1-1+\sum_{i=r+1}^{k} \hat{\alpha}_{i}^{2}(i)+o(1 / n)\right]>h_{3}\right) \\
& =\lim _{n \rightarrow \infty} \mathrm{P}\left(\sum_{i=r+1}^{k}\left(\sqrt{n}\left(\hat{\alpha}_{i}(i)-0\right)\right)^{2}>\frac{h_{3}}{\hat{\sigma}_{r}^{2}}\right) \\
& \geq \lim _{n \rightarrow \infty} \mathrm{P}\left(\sqrt{n}\left(\hat{\alpha}_{i}(i)-0\right)>\sqrt{\frac{h_{3}}{\hat{\sigma}_{r}^{2}}}\right) \\
& >0 .
\end{aligned}
$$

Usamos a normalidade assintótica de $\sqrt{n}\left(\hat{\alpha}_{i}(i)-0\right)$, que pode ser encontrada no Teorema 5.5.7 de Anderson (1994).

Proposição 2.17. Seja $\mathbb{X} u m A R(r)$ satisfazendo a Condição $2.3, k<r$, então

$$
\lim _{n \rightarrow \infty} \frac{\log L_{n}\left(\hat{\theta}_{r}\right)-\log L_{n}\left(\hat{\theta}_{k}\right)}{n}>0 \quad q . c .
$$

Demonstração. Tomando a série de Taylor para $\log L_{n, r}\left(\hat{\theta}_{k}\right)$ em $\hat{\theta}_{r}$ e usando (2.9), temos $\log L_{n, r}\left(\hat{\theta}_{k}\right)=\log L_{n, r}\left(\hat{\theta}_{r}\right)+\left(\hat{\theta}_{k}-\hat{\theta}_{r}\right) D_{\theta}^{1}\left(\log L_{n, r}\left(\hat{\theta}_{r}\right)\right)+\frac{1}{2}\left(\hat{\theta}_{k}-\hat{\theta}_{r}\right) D_{\theta}^{2}\left(\log L_{n, r}\left(\hat{\theta}_{r}\right)\right)\left(\hat{\theta}_{k}-\hat{\theta}_{r}\right)^{T}$ 
Como $L_{n, r}$ é maximizada por $\hat{\theta}_{r}$, temos que $D_{\theta}^{1}\left(\log L_{n, r}\left(\hat{\theta}_{r}\right)\right)=0$ e

$$
\begin{aligned}
\lim _{n \rightarrow \infty} \frac{\log L_{n, r}\left(\hat{\theta}_{r}\right)-\log L_{n, r}\left(\hat{\theta}_{k}\right)}{n} & =\lim _{n \rightarrow \infty} \frac{-\left(\hat{\theta}_{k}-\hat{\theta}_{r}\right) D_{\theta}^{2}\left(\log L_{n, r}\left(\hat{\theta}_{r}\right)\right)\left(\hat{\theta}_{k}-\hat{\theta}_{r}\right)^{T}}{2 n} \\
& =\frac{1}{2} \lim _{n \rightarrow \infty}\left(\hat{\theta}_{k}-\hat{\theta}_{r}\right) \frac{-D_{\theta}^{2}\left(\log L_{n, r}\left(\hat{\theta}_{r}\right)\right)}{n}\left(\hat{\theta}_{k}-\hat{\theta}_{r}\right)^{T} \\
& =\frac{1}{2} \lim _{n \rightarrow \infty}\left(\hat{\theta}_{k}-\hat{\theta}_{r}\right) A_{2}\left(\hat{\theta}_{k}-\hat{\theta}_{r}\right)^{T} \\
& =c \lim _{n \rightarrow \infty}\left\|\hat{\theta}_{k}-\hat{\theta}_{r}\right\| \\
& >0 .
\end{aligned}
$$

O resultado segue de (1.4), que $A_{2}$ é positiva definida (Lema 2.11) e que $\lim _{n \rightarrow \infty}\left\|\hat{\theta}_{k}-\hat{\theta}_{r}\right\|>0$ para $k<r$.

Embora a demonstração da Proposição anterior seja elegante, é utilizado diretamente o fato das derivadas de ordem 3 de $\log L_{n, k}$ serem nulas. Com isso, a técnica utilizada nessa demonstração não é aplicável no caso geral.

Teorema 2.18. Seja $\mathbb{X} u m$ AR(r) satisfazendo a Condição 2.3 e $\hat{r}_{\text {edc }}$ como na Definição 2.10.

(i) $\hat{r}_{\text {edc }}$ é fortemente consistente $\left(\hat{r}_{\text {edc }} \underset{q . c .}{\rightarrow} r\right)$ se

$$
\lim _{n \rightarrow \infty} \frac{c_{n}}{n}=0 \quad e \quad \liminf _{n \rightarrow \infty} \frac{c_{n}}{\log \log n}=\infty
$$

(ii) $\hat{r}_{e d c}$ é consistente $\left(\hat{r}_{e d c} \underset{P}{\rightarrow} r\right)$ se

$$
\lim _{n \rightarrow \infty} \frac{c_{n}}{n}=0 \quad e \quad \liminf _{n \rightarrow \infty} c_{n}=\infty .
$$

(iii) $\hat{r}_{e d c}$ é inconsistente se

$$
\limsup _{n \rightarrow \infty} c_{n}<\infty
$$

Demonstração. O conjunto dos possíveis valores de $\theta_{r}, \Theta_{r}$, definido em (2.3), é aberto e $\theta_{r} \in \Theta_{r}$. De Anderson (1994), temos que o estimador de máxima verossimilhança $\hat{\theta}_{k}$ é fortemente consistente para $k \geq r$, isto é, vale (1.10). As derivadas de $\log L_{n, k}$, (2.7) e (2.8) são contínuas em relação a $\theta$ e a $x_{1}^{n}$ e portanto são mensuráveis em relação a $x_{1}^{n}$. Temos de (2.9) que (1.11) é satisfeita. 
(i) Usando o Lema 2.11 temos que as condições satisfeitas para a aplicação do item (ii) do Teorema 1.6 e com isso temos (H2) satisfeita. A hipótese (H1) segue da Proposição 2.17. O resultado segue do item (i) do Teorema 1.5 .

(ii) Usando o Lema 2.11 e o Corolário 2.13, concluímos (H3) usando os Teoremas 1.6 e 1.7. Portanto temos o resultado a partir do Teorema 1.5 , item (ii).

(iii) De forma semelhante, usando o Teorema 2.16, temos (H4) e concluímos o resultado pelo item (iii) do Teorema 1.5 .

Corolário 2.19. Seja $\mathbb{X}$ um $A R(r)$ satisfazendo a Condição 2.3, $K \geq r$. Então o estimador $\hat{r}_{\text {bic2 }}$, como definido abaixo, é fortemente consistente.

$$
\hat{r}_{b i c 2}=\underset{k \in\{0, \ldots, K\}}{\operatorname{argmin}}\left\{-\log L_{n}\left(\hat{\theta}_{k}\right)+\frac{k}{2} \log n\right\}
$$

para $\log L_{n}\left(\hat{\theta}_{k}\right)$ como definida em (2.6).

Demonstração. $c_{n}=\log n / 2$ e satisfaz (2.19).

Corolário 2.20. Seja $\mathbb{X} u m A R(r)$ satisfazendo a Condição $2.3, K \geq r$. Então o estimador

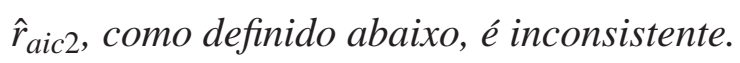

$$
\hat{r}_{\text {aic } 2}=\underset{k \in\{0, \ldots, K\}}{\operatorname{argmin}}\left\{-\log L_{n}\left(\hat{\theta}_{k}\right)+2 k\right\}
$$

para $\log L_{n}\left(\hat{\theta}_{k}\right)$ como definida em (2.6).

Demonstração. $\quad c_{n}=2$ e satisfaz (2.20).

Embora o estimador $\hat{r}_{\text {bic } 2}$ definido em (2.21) seja fortemente consistente, observou-se nas simulações numéricas que ele não apresenta performance satisfatória para casos em que $\sigma^{2} \neq 1$. Isso ocorre porque $-2 \log L_{n}\left(\hat{\theta}_{k}\right) / n$ coincide com o estimador de $\sigma^{2}$ supondo que a ordem seja $k$, e por isso as diferenças $\log L_{n}\left(\hat{\theta}_{k+1}\right)-\log L_{n}\left(\hat{\theta}_{k}\right)$ dependem, de certa forma, dessa grandeza. Os estimadores AIC, BIC e HQC resolvem esse problema aproximando $-\log L_{n}\left(\hat{\theta}_{k}\right) / n$ por $\log \left[-\log L_{n}\left(\hat{\theta}_{k}\right) / n\right]$. Entretanto, essa aproximação reduz a oscilação do estimador. Para solucionar isso, propomos o seguinte estimador de ordem. 
Corolário 2.21. Seja $\mathbb{X}$ um AR(r) satisfazendo a Condição 2.3, $K \geq r$. Então o estimador $\hat{r}_{\text {edc }}$, como definido abaixo, é fortemente consistente.

$$
\hat{r}_{e d c}=\underset{k \in\{0, \ldots, K\}}{\operatorname{argmin}}\left\{-\log L_{n}\left(\hat{\theta}_{k}\right)+\frac{k}{2} \hat{\sigma}_{K}^{2} \log n\right\}
$$

para $\log L_{n}\left(\hat{\theta}_{k}\right)$ como definida em (2.6) e $\hat{\sigma}_{K}^{2}$ o estimador de $\sigma^{2}$.

Demonstração. $c_{n}=\hat{\sigma}_{K}^{2} \log n / 2$ e satisfaz (2.19).

Vale ressaltar que o fator $\hat{\sigma}_{K}^{2}$ é utilizado para se obter uma aproximação da grandeza de $\sigma^{2}$ o que não requer precisão, e portanto não limita de forma substancial o valor de $K$.

\subsubsection{Simulações numéricas}

Os estimadores considerados nas simulações são, para uma amostra $x_{1}^{n}$,

$$
\begin{gathered}
\hat{r}_{a i c}=\underset{k=0, \ldots, K}{\operatorname{argmin}}\{A I C(k)\}, \\
\hat{r}_{b i c}=\underset{k=0, \ldots, K}{\operatorname{argmin}}\{B I C(k)\}, \\
\hat{r}_{h q c}=\underset{k=0, \ldots, K}{\operatorname{argmin}}\{H Q C(k)\} \quad e \\
\hat{r}_{e d c}=\underset{k=0, \ldots, K}{\operatorname{argmin}}\{\operatorname{EDC}(k)\} .
\end{gathered}
$$

Onde,

$$
\begin{gathered}
A I C(k)=n \log \left(\sum_{t=k+1}^{n} \frac{\left[x_{t}-\left(x_{t-1} \hat{\alpha}_{1}+\cdots+x_{t-k} \hat{\alpha}_{k}\right)\right]^{2}}{n}\right)+2 k, \\
B I C(k)=n \log \left(\sum_{t=k+1}^{n} \frac{\left[x_{t}-\left(x_{t-1} \hat{\alpha}_{1}+\cdots+x_{t-k} \hat{\alpha}_{k}\right)\right]^{2}}{n}\right)+k \log n \\
H Q C(k)=n \log \left(\sum_{t=k+1}^{n} \frac{\left[x_{t}-\left(x_{t-1} \hat{\alpha}_{1}+\cdots+x_{t-k} \hat{\alpha}_{k}\right)\right]^{2}}{n}\right)+2 k \log \log n \\
E D C(k)=\sum_{t=k+1}^{n}\left[x_{t}-\left(x_{t-1} \hat{\alpha}_{1}+\cdots+x_{t-k} \hat{\alpha}_{k}\right)\right]^{2}+k \hat{\sigma}_{K}^{2} \log n e
\end{gathered}
$$




$$
\hat{\sigma}_{K}^{2}=\frac{\sum_{t=K+1}^{n}\left[x_{t}-\left(x_{t-1} \hat{\alpha}_{1}+\cdots+x_{t-K} \hat{\alpha}_{K}\right)\right]^{2}}{n} .
$$

Os $\hat{\alpha}_{i}$ são os estimadores de máxima verossimilhança para os parâmetros $\alpha_{i}$. Não há fórmula explícita para a estimação dos $\hat{\alpha}_{i}$. Eles são a solução do seguinte sistema linear.

$$
\begin{gathered}
{\left[\begin{array}{ccc}
\hat{R}(1,1) & \ldots & \hat{R}(1, k) \\
\vdots & & \vdots \\
\hat{R}(k, 1) & \ldots & \hat{R}(k, k)
\end{array}\right]\left[\begin{array}{c}
\hat{\alpha}(1) \\
\vdots \\
\hat{\alpha}(k)
\end{array}\right]=\left[\begin{array}{c}
\hat{R}(0,1) \\
\vdots \\
\hat{R}(0, k)
\end{array}\right]} \\
\hat{R}(i, j)=\frac{1}{n} \sum_{t=k+1}^{n} X_{t-i} X_{t-j}
\end{gathered}
$$

Ressalta-se que foram utilizadas as definições propostas por Shibata (1976) e Hannan \& Quinn (1979) para os estimadores baseados nos critérios AIC, BIC e HQC.

Na Tabela 2.1 estão os resultados de simulações numéricas para modelos AR com ordem $r \in\{1,3,10,50\}$ e desvio padrão $\sigma=0,1$. Para cada caso foram realizados 100 simulações. Os valores iniciais, de 1 até $K$, foram gerados aleatoriamente usando a distribuição $\mathscr{N}\left(0, \sigma^{2}\right)$. A coluna $r$ representa a ordem e $n$ é o tamanho da amostra, que foi escolhida empiricamente para melhor comparação entre os estimadores. As colunas " $<$ ", "=" e " $>$ " representam respectivamente as quantidades de casos que o estimador "subestimou a ordem", "acertou a ordem" ou "superestimou a ordem". Nas Tabelas 2.2, 2.3 e 2.4 temos respectivamente os casos onde $\sigma=1, \sigma=10$ e $\sigma=1000$. Para $r=1,3,10$ foi considerado $K=20$ e para $r=50$ foi considerado $K=100$. Em todos os casos simulados foi considerado $\alpha_{i}=0$ para $i<r$ e $\alpha_{r}=0,5$.

Observa-se que o EDC apresentou performance superior ao HQC e AIC em todos os casos. Comparado com o BIC, o EDC apresenta performance ligeiramente inferior em casos simples, onde $r \leq 3$, e superior para casos mais complexos, $r \geq 10$. Isso sugere que em casos de maior complexidade o EDC apresente performance relativa ainda maior. Ressaltase que simulações mais detalhadas, modificando-se as escolhas de $\theta$ e $n$, podem nos levar a conclusões mais precisas. Entretanto, estes aspectos não são os objetivos principais deste trabalho. 
Tabela 2.1: caso $\sigma=0,1$

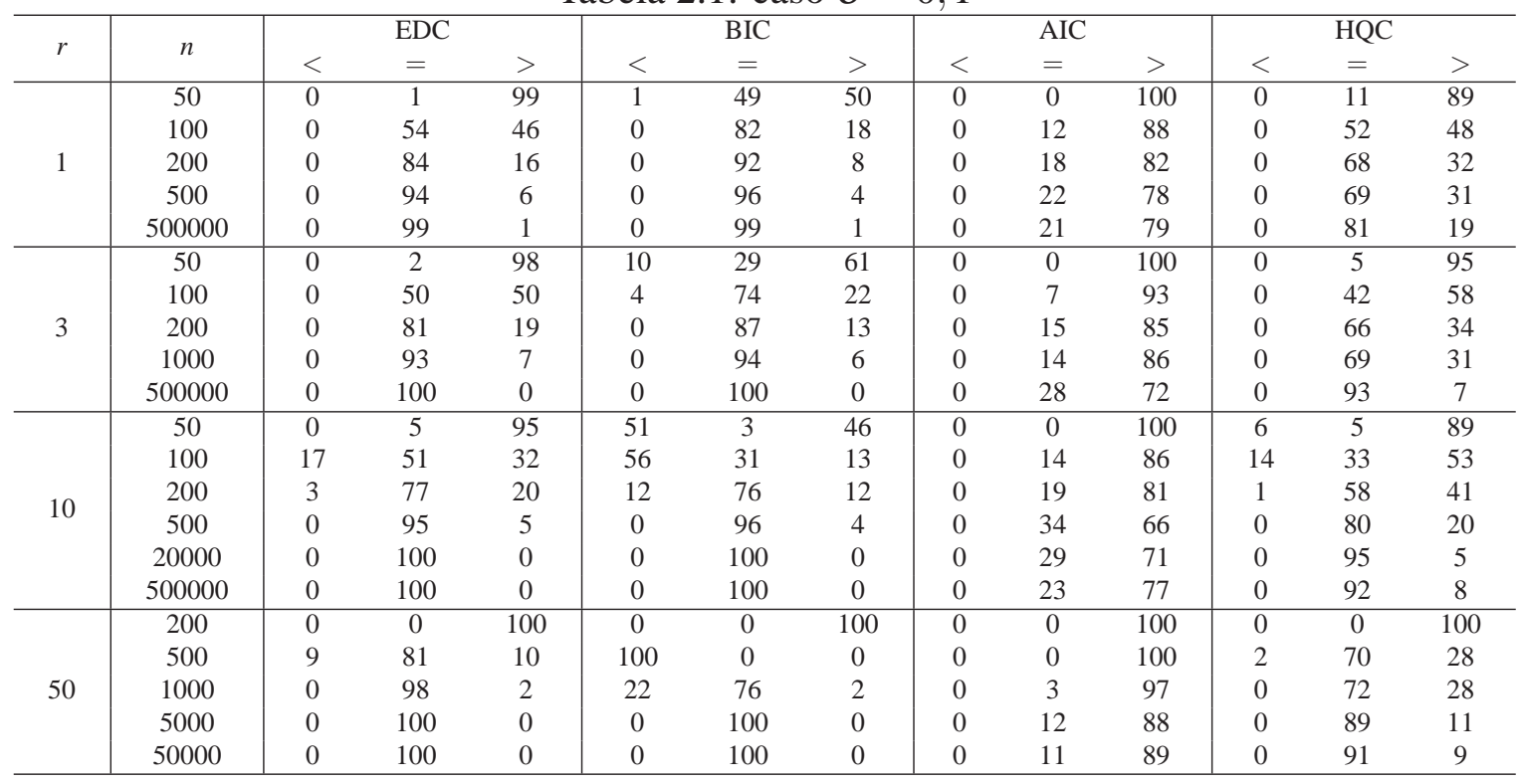

Tabela 2.2: caso $\sigma=1$

\begin{tabular}{|c|c|c|c|c|c|c|c|c|c|c|c|c|c|}
\hline \multirow{2}{*}{$r$} & \multirow{2}{*}{$n$} & \multicolumn{3}{|c|}{ EDC } & \multicolumn{3}{|c|}{ BIC } & \multicolumn{3}{|c|}{ AIC } & \multicolumn{3}{|c|}{ HQC } \\
\hline & & $<$ & $=$ & $>$ & $<$ & $=$ & $>$ & $<$ & $=$ & $>$ & $<$ & $=$ & $>$ \\
\hline \multirow{5}{*}{1} & 100 & 0 & 47 & 53 & 0 & 87 & 13 & 0 & 4 & 96 & 0 & 43 & 57 \\
\hline & 200 & 0 & 84 & 16 & 0 & 92 & 8 & 0 & 18 & 82 & 0 & 68 & 32 \\
\hline & 500 & 0 & 93 & 7 & 0 & 95 & 5 & 0 & 13 & 87 & 0 & 81 & 19 \\
\hline & 50000 & 0 & 100 & 0 & 0 & 100 & 0 & 0 & 19 & 81 & 0 & 92 & 8 \\
\hline & 500000 & 0 & 100 & 0 & 0 & 100 & 0 & 0 & 17 & 83 & 0 & 95 & 5 \\
\hline \multirow{4}{*}{3} & 100 & 0 & 50 & 50 & 0 & 77 & 23 & 0 & 7 & 93 & 0 & 46 & 54 \\
\hline & 200 & 0 & 81 & 19 & 0 & 87 & 13 & 0 & 15 & 85 & 0 & 66 & 34 \\
\hline & 500 & 0 & 94 & 6 & 0 & 94 & 6 & 0 & 23 & 77 & 0 & 74 & 26 \\
\hline & 500000 & 0 & 99 & 1 & 0 & 99 & 1 & 0 & 27 & 73 & 0 & 82 & 18 \\
\hline \multirow{6}{*}{10} & 100 & 16 & 42 & 42 & 54 & 35 & 11 & 0 & 7 & 93 & 11 & 35 & 54 \\
\hline & 200 & 3 & 77 & 20 & 12 & 76 & 12 & 0 & 19 & 81 & 1 & 58 & 41 \\
\hline & 500 & 0 & 89 & 11 & 0 & 89 & 11 & 0 & 16 & 84 & 0 & 65 & 35 \\
\hline & 10000 & 0 & 99 & 1 & 0 & 99 & 1 & 0 & 26 & 74 & 0 & 83 & 17 \\
\hline & 500000 & 0 & 100 & 0 & 0 & 100 & 0 & 0 & 28 & 72 & 0 & 90 & 10 \\
\hline & 1000000 & 0 & 100 & 0 & 0 & 100 & 0 & 0 & 20 & 80 & 0 & 94 & 6 \\
\hline \multirow{5}{*}{50} & 500 & 12 & 65 & 23 & 99 & 0 & 1 & 0 & 1 & 99 & 4 & 57 & 39 \\
\hline & 1000 & 0 & 94 & 6 & 23 & 74 & 3 & 0 & 1 & 99 & 0 & 67 & 33 \\
\hline & 5000 & 0 & 99 & 1 & 0 & 99 & 1 & 0 & 5 & 95 & 0 & 90 & 10 \\
\hline & 20000 & 0 & 99 & 1 & 0 & 99 & 1 & 0 & 10 & 90 & 0 & 88 & 12 \\
\hline & 50000 & 0 & 100 & 0 & 0 & 100 & 0 & 0 & 11 & 89 & 0 & 79 & 21 \\
\hline
\end{tabular}


Tabela 2.3: caso $\sigma=10$

\begin{tabular}{|c|c|c|c|c|c|c|c|c|c|c|c|c|c|}
\hline \multirow{2}{*}{$r$} & \multirow{2}{*}{$n$} & \multicolumn{3}{|c|}{ EDC } & \multicolumn{3}{|c|}{$\mathrm{BIC}$} & \multicolumn{3}{|c|}{ AIC } & \multicolumn{3}{|c|}{ HQC } \\
\hline & & $<$ & $=$ & $>$ & $<$ & $=$ & $>$ & $<$ & $=$ & $>$ & $<$ & $=$ & $>$ \\
\hline \multirow{6}{*}{1} & 50 & 0 & 2 & 98 & 4 & 47 & 49 & 0 & 0 & 100 & 0 & 7 & 93 \\
\hline & 100 & 0 & 54 & 46 & 0 & 83 & 17 & 0 & 5 & 95 & 0 & 47 & 53 \\
\hline & 200 & 0 & 84 & 16 & 0 & 92 & 8 & 0 & 18 & 82 & 0 & 68 & 32 \\
\hline & 500 & 0 & 95 & 5 & 0 & 98 & 2 & 0 & 13 & 87 & 0 & 76 & 24 \\
\hline & 10000 & 0 & 100 & 0 & 0 & 100 & 0 & 0 & 18 & 82 & 0 & 85 & 15 \\
\hline & 500000 & 0 & 100 & 0 & 0 & 100 & 0 & 0 & 24 & 76 & 0 & 92 & 8 \\
\hline \multirow{6}{*}{3} & 50 & 0 & 2 & 98 & 12 & 30 & 58 & 0 & 0 & 100 & 0 & 5 & 95 \\
\hline & 100 & 0 & 49 & 51 & 3 & 72 & 25 & 0 & 9 & 91 & 0 & 41 & 59 \\
\hline & 200 & 0 & 81 & 19 & 0 & 87 & 13 & 0 & 15 & 85 & 0 & 66 & 34 \\
\hline & 500 & 0 & 93 & 7 & 0 & 94 & 6 & 0 & 17 & 83 & 0 & 75 & 25 \\
\hline & 5000 & 0 & 100 & 0 & 0 & 100 & 0 & 0 & 15 & 85 & 0 & 83 & 17 \\
\hline & 500000 & 0 & 99 & 1 & 0 & 99 & 1 & 0 & 21 & 79 & 0 & 90 & 10 \\
\hline \multirow{7}{*}{10} & 50 & 1 & 8 & 91 & 36 & 6 & 58 & 1 & 1 & 98 & 4 & 6 & 90 \\
\hline & 100 & 7 & 59 & 34 & 41 & 48 & 11 & 0 & 9 & 91 & 5 & 38 & 57 \\
\hline & 200 & 3 & 77 & 20 & 12 & 76 & 12 & 0 & 19 & 81 & 1 & 58 & 41 \\
\hline & 500 & 0 & 92 & 8 & 0 & 93 & 7 & 0 & 23 & 77 & 0 & 71 & 29 \\
\hline & 1000 & 0 & 96 & 4 & 0 & 97 & 3 & 0 & 21 & 79 & 0 & 81 & 19 \\
\hline & 5000 & 0 & 100 & 0 & 0 & 100 & 0 & 0 & 32 & 68 & 0 & 88 & 12 \\
\hline & 500000 & 0 & 99 & 1 & 0 & 99 & 1 & 0 & 20 & 80 & 0 & 92 & 8 \\
\hline \multirow{6}{*}{50} & 200 & 0 & 0 & 100 & 0 & 0 & 100 & 0 & 0 & 100 & 0 & 0 & 100 \\
\hline & 500 & 4 & 79 & 17 & 98 & 2 & 0 & 0 & 3 & 97 & 3 & 62 & 35 \\
\hline & 1000 & 0 & 94 & 6 & 28 & 70 & 2 & 0 & 4 & 96 & 0 & 70 & 30 \\
\hline & 2000 & 0 & 97 & 3 & 0 & 99 & 1 & 0 & 12 & 88 & 0 & 78 & 22 \\
\hline & 5000 & 0 & 99 & 1 & 0 & 99 & 1 & 0 & 11 & 89 & 0 & 85 & 15 \\
\hline & 100000 & 0 & 99 & 1 & 0 & 99 & 1 & 0 & 8 & 92 & 0 & 94 & 6 \\
\hline
\end{tabular}

Tabela 2.4: caso $\sigma=1000$

\begin{tabular}{|c|c|c|c|c|c|c|c|c|c|c|c|c|c|}
\hline \multirow{2}{*}{$r$} & \multirow{2}{*}{$n$} & \multicolumn{3}{|c|}{ EDC } & \multicolumn{3}{|c|}{ BIC } & \multicolumn{3}{|c|}{ AIC } & \multicolumn{3}{|c|}{ HQC } \\
\hline & & $<$ & $=$ & $>$ & $<$ & $=$ & $>$ & $<$ & $=$ & $>$ & $<$ & $=$ & $>$ \\
\hline \multirow{5}{*}{1} & 50 & 0 & 3 & 97 & 6 & 52 & 42 & 0 & 0 & 100 & 1 & 10 & 89 \\
\hline & 100 & 0 & 59 & 41 & 0 & 94 & 6 & 0 & 8 & 92 & 0 & 55 & 45 \\
\hline & 500 & 0 & 95 & 5 & 0 & 96 & 4 & 0 & 16 & 84 & 0 & 76 & 24 \\
\hline & 20000 & 0 & 100 & 0 & 0 & 100 & 0 & 0 & 16 & 84 & 0 & 84 & 16 \\
\hline & 100000 & 2 & 98 & 0 & 0 & 100 & 0 & 0 & 20 & 80 & 0 & 90 & 10 \\
\hline \multirow{5}{*}{3} & 100 & 0 & 47 & 53 & 3 & 80 & 17 & 0 & 5 & 95 & 0 & 41 & 59 \\
\hline & 200 & 0 & 81 & 19 & 0 & 87 & 13 & 0 & 15 & 85 & 0 & 66 & 34 \\
\hline & 1000 & 0 & 99 & 1 & 0 & 99 & 1 & 0 & 24 & 76 & 0 & 80 & 20 \\
\hline & 10000 & 0 & 99 & 1 & 0 & 99 & 1 & 0 & 12 & 88 & 0 & 83 & 17 \\
\hline & 100000 & 6 & 94 & 0 & 0 & 98 & 2 & 0 & 23 & 77 & 0 & 90 & 10 \\
\hline \multirow{3}{*}{10} & 50 & 2 & 3 & 95 & 41 & 5 & 54 & 0 & 1 & 99 & 5 & 3 & 92 \\
\hline & 10000 & 0 & 100 & 0 & 0 & 100 & 0 & 0 & 28 & 72 & 0 & 78 & 22 \\
\hline & 100000 & 1 & 99 & 0 & 0 & 100 & 0 & 0 & 25 & 75 & 0 & 86 & 14 \\
\hline \multirow{6}{*}{50} & 200 & 0 & 0 & 100 & 0 & 0 & 100 & 0 & 0 & 100 & 0 & 0 & 100 \\
\hline & 500 & 9 & 67 & 24 & 100 & 0 & 0 & 0 & 1 & 99 & 5 & 55 & 40 \\
\hline & 1000 & 0 & 98 & 2 & 24 & 75 & 1 & 0 & 4 & 96 & 0 & 79 & 21 \\
\hline & 2000 & 0 & 99 & 1 & 0 & 99 & 1 & 0 & 5 & 95 & 0 & 81 & 19 \\
\hline & 5000 & 0 & 100 & 0 & 0 & 100 & 0 & 0 & 11 & 89 & 0 & 93 & 7 \\
\hline & 50000 & 0 & 100 & 0 & 0 & 100 & 0 & 0 & 9 & 91 & 0 & 90 & 10 \\
\hline
\end{tabular}




\subsection{Modelo Autoregressivo de Heteroscedasticidade Condicional (ARCH)}

Processos Autoregressivos de Heteroscedasticidade Condicional (ARCH) foram propostos originalmente por Engle (1982) como uma melhor alternativa para modelagem de séries temporais em cenários econômicos. Desde então, modelos ARCH e variações vêm sendo utilizados com sucesso em econometria na modelagem de taxas de inflação (Engle 1982), séries temporais em mercado de câmbio (Domowitz \& Hakkio 1985), valores de ativos (Bollerslev, Chou \& Kroner 1992), dentre outros.

A consistência forte e normalidade assintótica dos estimadores para o caso ARCH foi estabelecida por Weiss (1986). Muito embora não existam trabalhos sobre a consistência de estimadores de ordem para modelos da família $\mathrm{ARCH}$, os critérios de informação AIC e BIC vêm sendo utilizados sem qualquer formalização (Hughes, King \& Kwek 2004).

Nessa seção a classe de estimadores EDC é definida para processo ARCH e a consistência forte é estabelecida em função do termo de penalidade. Como consequência imediata, a consistência forte do estimador de ordem BIC é demonstrada.

Processos ARCH é um caso particular dos processos BEKK-GARCH, que é objeto da próxima seção. Todavia, as demonstrações para o caso ARCH utilizam condições diferentes das exigidas para o caso geral, e por isso são mantidas.

\subsubsection{Definições}

No que segue, é apresentada a definição para modelos ARCH seguida de condições de ergodicidade e regularidade que são necessárias para o estabelecimento dos próximos resultados. A propriedade básica de processos ARCH é que o desvio padrão da inovação depende dos últimos $r$ valores do processo.

Definição 2.22. Uma sequência de variáveis aleatórias $\mathbb{X}=\left\{X_{t}\right\}_{t \in \mathbb{N}}$ é um processo Autoregressivo de Heteroscedasticidade Condicional (ARCH) de ordem $r \in \mathbb{N}$, denotado por ARCH(r), se satisfaz as condições abaixo. 
(i) Para todo $t \in \mathbb{N}$,

$$
X_{t}=\varepsilon_{t}\left(h_{t}\right)^{\frac{1}{2}}
$$

(ii) $h_{t}=h\left(\alpha_{0}^{r}, X_{t-1}^{t-r}\right)=\alpha_{0}+\alpha_{1} X_{t-1}^{2}+\cdots+\alpha_{r} X_{t-r}^{2}, \theta_{r}=\left(\alpha_{0}, \ldots, \alpha_{r}\right) \in \mathbb{R}^{r+1}$ e $\alpha_{0}, \alpha_{r}>0$.

(iii) $\left\{\varepsilon_{t}\right\}_{t \in \mathbb{N}}$ é uma sequência de variáveis aleatórias i.i.d. $\mathscr{N}(0,1)$.

Condição 2.5 (Ergodicidade). Para $\mathbb{X} u m \operatorname{ARCH}(r)$, e $\theta_{r}=\left(\alpha_{0}, \ldots, \alpha_{r}\right) \in \mathbb{R}^{r+1}$ seus respectivos parâmetros, assumimos que

$$
\sum_{i=1}^{r} \alpha_{i}<1
$$

Condição 2.6 (Regularidade). Para $\mathbb{X} u m A R C H(r)$, então

(i)

$$
E\left(X_{t}^{4}\right)<\infty
$$

(ii) $\alpha_{i}>0$ para todo $i \in\{1, \ldots, r\}$.

(iii) Existe um intervalo conhecido, $I=[c, d]$, tal que $\alpha_{0} \in I^{\circ}=(c, d)$ e $c>0$.

Usando a Condição 2.5, Francq \& Zakoian (2010) (Teorema 2.5, página 37) demonstra que $X_{t}$ admite momentos estacionários finitos de ordem 2. O Teorema 2.9 (página 45) da mesma referência provê condições para a existência de momentos pares maiores que 2. O uso do item (ii) da Condição 2.6 dispensa a exigência de momentos de ordem superiores. Em trabalhos futuros, deve ser avaliado a possibilidade de adequar a técnica para enfraquecer essa exigência. O item (iii) é a particularização da condição exigida em Jeantheau (1998) e Comte \& Lieberman (2003).

\section{Principais propriedades do processo $\mathrm{ARCH}$}

(a) $E\left(X_{t}\right)=0$.

$$
E\left(X_{t}\right)=E\left(E\left(X_{t} \mid \mathscr{F}_{t-1}\right)\right)=E(0)=0 .
$$

(b) $E\left(X_{t} X_{t-k}\right)=0$, se $k \geq 1$

$$
E\left(X_{t} X_{t-k}\right)=E\left(E\left(X_{t} X_{t-k} \mid \mathscr{F}_{t-1}\right)\right)=E\left(X_{t-k} E\left(X_{t} \mid \mathscr{F}_{t-1}\right)\right)=0 .
$$


(c) $h_{t} \geq \alpha_{0}+\alpha_{1}^{i} X_{t-i}^{2} \prod_{p=1}^{i-1} \varepsilon_{t-p}^{2} \geq \alpha_{1}^{i} X_{t-i}^{2} \prod_{p=1}^{i-1} \varepsilon_{t-p}^{2}$, se $i \geq 1$. Basta utilizar um argumento indutivo para

$$
\begin{aligned}
h_{t} & =\alpha_{0}+\alpha_{1} X_{t-1}^{2}+\cdots+\alpha_{r} X_{t-r}^{2} \\
& \geq \alpha_{0}+\alpha_{1} X_{t-1}^{2} \\
& =\alpha_{0}+\alpha_{1} \varepsilon_{t-1}^{2} h_{t-1} .
\end{aligned}
$$

(d) Um ARCH(r) $\mathbb{X}$ pode ser imerso em uma sequência de modelos aninhados $\mathbb{M}=\bigcup_{k=0}^{\infty} M_{k}$, tomando $\Theta_{k}=I \times[0,1]^{k}$ e, para $\theta_{k}=\left(\alpha_{0}, \cdots, \alpha_{k}\right) \in \Theta_{k}$

$$
f\left(x_{1}^{n}, \theta_{k}\right)=C\left(x_{1}^{k}\right) \prod_{t=1+k}^{n} \frac{1}{\sqrt{2 \pi h_{t}}} e^{-\frac{X_{t}^{2}}{2 h_{t}}} .
$$

Funções $\log L_{n, k}$ e suas derivadas

A log-verossimilhança de $m_{k}=f\left(X_{1}^{n}, \theta_{k}\right) \in M_{k}$ dada por

$$
\begin{aligned}
\log \left(\prod_{t=1+k}^{n} f\left(X_{t} \mid X_{t-k-1}^{t-1}\right)\right)+C_{1}\left(X_{1}^{k}\right) & =\sum_{t=1+k}^{n} \log \left(\frac{1}{\sqrt{2 \pi h_{t}}} e^{-\frac{X_{t}^{2}}{2 h_{t}}}\right)+C_{1}\left(X_{1}^{k}\right) \\
& =\sum_{t=1+k}^{n}\left\{-\frac{X_{t}^{2}}{2 h_{t}}-\frac{1}{2} \log h_{t}-\log \sqrt{2 \pi}\right\}+C_{1}\left(X_{1}^{k}\right) \\
& =\sum_{t=1+k}^{n}\left\{-\frac{X_{t}^{2}}{2 h_{t}}-\frac{1}{2} \log h_{t}\right\}-(n-k) \log \sqrt{2 \pi}+C_{1}\left(X_{1}^{k}\right)
\end{aligned}
$$

Definimos abaixo o logaritmo de $L_{n, k}$, que satisfaz a Definição 1.3.

$$
\log L_{n, k}(\theta)=\sum_{t=1+k}^{n}\left\{-\frac{X_{t}^{2}}{2 h_{t}}-\frac{1}{2} \log h_{t}\right\} .
$$

Na literatura (exemplo em Bollerslev (1986) e Engle (1982)) a função (2.24) é assumida diretamente como a $\log$-verossimilhança. Para $i, j, l \in\{1, \cdots, k\}$,

$$
\begin{aligned}
\frac{\partial \log L_{n, k}(\theta)}{\partial \alpha_{i}} & =\sum_{t=1+k}^{n}\left\{\frac{X_{t}^{2}}{2 h_{t}^{2}} \frac{\partial h_{t}}{\partial \alpha_{i}}-\frac{1}{2 h_{t}} \frac{\partial h_{t}}{\partial \alpha_{i}}\right\} \\
& =\sum_{t=1+k}^{n}\left\{\frac{X_{t-i}^{2}}{2 h_{t}}\left(\frac{X_{t}^{2}}{h_{t}}-1\right)\right\} .
\end{aligned}
$$




$$
\begin{aligned}
\frac{\partial^{2} \log L_{n, k}(\theta)}{\partial \alpha_{i} \alpha_{j}} & =\sum_{t=1+k}^{n}\left\{-\frac{1}{2 h_{t}^{2}} \frac{\partial h_{t}}{\partial \alpha_{i}} \frac{\partial h_{t}}{\partial \alpha_{j}} \frac{X_{t}^{2}}{h_{t}}+\left(\frac{X_{t}^{2}}{h_{t}}-1\right) \frac{\partial}{\partial \alpha_{j}}\left\{\frac{1}{2 h_{t}} \frac{\partial h_{t}}{\partial \alpha_{i}}\right\}\right\} \\
& =\sum_{t=1+k}^{n}\left\{-\frac{X_{t-i}^{2} X_{t-j}^{2} X_{i}^{2}}{2 h_{i}^{3}}-\frac{X_{t-i}^{2} X_{t-j}^{2} X_{i}^{2}}{2 h_{i}^{3}}+\frac{X_{t-i}^{2} X_{t-j}^{2}}{2 h_{i}^{2}}\right\} \\
& =\sum_{t=1+k}^{n}\left\{-\frac{2 X_{t-i}^{2} X_{t-j}^{2} X_{i}^{2}}{2 h_{i}^{3}}+\frac{X_{t-i}^{2} X_{t-j}^{2}}{2 h_{i}^{2}}\right\} .
\end{aligned}
$$

$$
\begin{aligned}
\frac{\partial^{3} \log L_{n, k}(\theta)}{\partial \alpha_{i} \alpha_{j} \alpha_{l}}= & \sum_{t=1+k}^{n}\left\{\frac{1}{h_{t}^{3}} \frac{\partial h_{t}}{\partial \alpha_{l}}\left(\frac{\partial h_{t}}{\partial \alpha_{i}} \frac{\partial h_{t}}{\partial \alpha_{j}} \frac{X_{t}^{2}}{h_{t}}\right)\right. \\
& -\frac{1}{2 h_{t}^{2}}\left(\frac{\partial}{\partial \alpha_{l}}\left\{\frac{\partial h_{t}}{\partial \alpha_{i}} \frac{\partial h_{t}}{\partial \alpha_{j}}\right\} \frac{X_{t}^{2}}{h_{t}}+\frac{\partial h_{t}}{\partial \alpha_{i}} \frac{\partial h_{t}}{\partial \alpha_{j}}\left(-\frac{X_{t}^{2}}{h_{t}^{2}} \frac{\partial h_{t}}{\partial \alpha_{l}}\right)\right) \\
& \left.+\left(-\frac{X_{t}^{2}}{h_{t}^{2}} \frac{\partial h_{t}}{\partial \alpha_{l}}\right) \frac{\partial}{\partial \alpha_{j}}\left\{\frac{1}{2 h_{t}} \frac{\partial h_{t}}{\partial \alpha_{i}}\right\}+\left(\frac{X_{t}^{2}}{h_{t}}-1\right) \frac{\partial^{2}}{\partial \alpha_{j} \alpha_{l}}\left\{\frac{1}{2 h_{t}} \frac{\partial h_{t}}{\partial \alpha_{k}}\right\}\right\} \\
= & \sum_{t=1+k}^{n}\left\{\frac{X_{t-i}^{2} X_{t-j}^{2} X_{t-l}^{2}}{h_{t}^{3}} \frac{X_{t}^{2}}{h_{t}}\right. \\
& \left.+\frac{X_{t-i}^{2} X_{t-j}^{2} X_{t-l}^{2} \frac{X_{t}^{2}}{h_{t}}+\frac{X_{t-i}^{2} X_{t-j}^{2} X_{t-l}^{2}}{2 h_{t}^{3}} \frac{X_{t}^{2}}{h_{t}}}{}+\left(\frac{X_{t}^{2}}{h_{t}}-1\right) \frac{X_{t-i}^{2} X_{t-j}^{2} X_{t-l}^{2}}{h_{t}^{3}}\right\} \\
= & \sum_{t=1+k}^{n}\left\{\frac{2 X_{t-i}^{2} X_{t-j}^{2} X_{t-l}^{2} X_{t}^{2}}{h_{t}^{4}}+\left(\frac{X_{t}^{2}}{h_{t}}-1\right) \frac{X_{t-i}^{2} X_{t-j}^{2} X_{t-l}^{2}}{h_{t}^{3}}\right\} \\
= & \sum_{t=1+k}^{n}\left\{\frac{3 X_{t-i}^{2} X_{t-j}^{2} X_{t-l}^{2} X_{t}^{2}}{h_{t}^{4}}-\frac{X_{t-i}^{2} X_{t-j}^{2} X_{t-l}^{2}}{h_{t}^{3}}\right\} .
\end{aligned}
$$

Convencionamos $0 / 0=0 / \infty=0$. Para $i, j, l \in\{0, \ldots, k\}$ (2.25), (2.26) e (2.27) valem se definirmos " $X_{t-0}^{2}:=1 "$.

Definição 2.23. Para $\left\{X_{t}\right\}_{t \in \mathbb{N}}$ um $A R C H(r), k \geq r, \log L_{n}\left(\hat{\theta}_{k}\right)$ como definido em (2.24), $\theta_{r} \in \Theta_{k} \subseteq \mathbb{R}^{k+1}$, definimos

$$
m(i, j)=-E\left(\frac{\partial^{2} \log L_{n}\left(\theta_{r}\right)}{\partial \alpha_{i} \alpha_{j}}\right)
$$




\subsubsection{Consistência do estimador de ordem de dependência}

Os resultados desenvolvidos nessa seção seguem a mesma técnica utilizada para a definição do estimador EDC em processos AR. Para fazer isso, os processos ARCH são inseridos no contexto de cadeias de Markov para obtenção da Lei Forte dos Grandes Números. Foi necessário a utilização de resultados desenvolvidos para Martingales para a aplicação da Lei do Logaritmo Iterado.

Na definição abaixo o estimador de ordem EDC é definido de forma particular para processos ARCH. O Teorema 2.47 provê condições para $c_{n}$ que definem a classe de estimadores EDC fortemente consistentes.

Definição 2.24. Para $\mathbb{X} u m A R C H(r)$ e $K \geq r$, definimos o estimador EDC de $r$ por

$$
\hat{r}_{e d c}=\underset{k \in\{0, \ldots, K\}}{\operatorname{argmin}}\left\{-\log L_{n, k}\left(\hat{\theta}_{k}\right)+(k+1) c_{n}\right\}
$$

para $\log L_{n, k}\left(\hat{\theta}_{k}\right)$ como definida em (2.24) $e\left\{c_{n}\right\}_{n \in \mathbb{N}}$ uma sequência de números positivos.

O processo de imersão de processos ARCH em cadeias de Markov foi proposto, em caso mais geral, por Boussama (1998). A partir disso vêm sendo utilizado por outros autores, tais como Comte \& Lieberman (2003) e Hafner \& Preminger (2009a, 2009b). Abaixo segue a particularização desse resultado.

Definição 2.25. Seja $\mathbb{X}=\left\{X_{t}\right\}_{t \in \mathbb{N}}$ um ARCH(r). A cadeia de Markov $k$-derivada de $\mathbb{X}$ é $o$ processo $\mathbb{Y}=\left\{Y_{t}\right\}_{t \in \mathbb{N}}$ com valores em $\mathbb{R}^{k}$,

$$
Y_{t}=\left(X_{t}, X_{t-1}, \ldots, X_{t-k+1}\right)^{\prime}
$$

Teorema 2.26 (Boussama (1998)). Seja $\left\{X_{t}\right\}_{t \in \mathbb{N}} u m A R C H(r), k \geq r$, satisfazendo a Condição 2.5, então $\mathbb{Y}$, a cadeia de Markov k-derivada de $\mathbb{X}$, é Harris positiva e geometricamente ergódica.

O Teorema 2.27 estabelece certa regularidade no comportamento assintótico de alguns objetos, que são necessários para o desenvolvimento dos próximos resultados. 
Teorema 2.27. Seja $\left\{X_{t}\right\}_{t \in \mathbb{N}}$ um ARCH(r), $k \geq r, \log L_{n, k}$ como definido em (2.24), $\hat{\theta}_{k} \in$ $\Theta_{k} \subseteq \mathbb{R}^{k+1}$ satisfazendo as Condições 2.5 e 2.6 e $\dot{\theta}=s \theta_{r}+(1-s) \hat{\theta}_{k}, s \in[0,1]$, então

(i)

$$
\lim _{n \rightarrow \infty}-\frac{D_{\theta}^{2} \log L_{n, k}(\dot{\theta})}{n}=A_{2} \quad \text { q.c. }
$$

Onde

$$
A_{2}=\left[\begin{array}{ccc}
m(0,0) & \ldots & m(0, k) \\
\vdots & \ddots & \vdots \\
m(k, 0) & \ldots & m(k, k)
\end{array}\right]
$$

(ii) $A_{2}$ é positiva definida.

(iii) Existe $c \in(0, \infty)$, tal que

$$
\lim _{n \rightarrow \infty}\left|\frac{\frac{\partial^{3} \log L_{n, k}(\dot{\theta})}{\partial \alpha_{i} \alpha_{j} \alpha_{l}}}{n}\right| \leq c
$$

Demonstração. (i) Usando a definição de $h_{t}$, temos que

$$
\begin{aligned}
E\left\{\left|-\frac{1}{2 h_{t}^{2}} \frac{\partial h_{t}}{\partial \alpha_{i}} \frac{\partial h_{t}}{\partial \alpha_{j}} \frac{X_{t}^{2}}{h_{t}}+\left(\frac{X_{t}^{2}}{h_{t}}-1\right) \frac{\partial}{\partial \alpha_{j}}\left\{\frac{1}{2 h_{t}} \frac{\partial h_{t}}{\partial \alpha_{i}}\right\}\right|\right\} & =E\left\{\left|-\frac{X_{t-i}^{2} X_{t-j}^{2} X_{t}^{2}}{2 h_{t}^{3}}-\frac{X_{t-i}^{2} X_{t-j}^{2} X_{t}^{2}}{2 h_{t}^{3}}+\frac{X_{t-i}^{2} X_{t-j}^{2}}{2 h_{t}^{2}}\right|\right\} \\
& \leq E\left\{\frac{2 X_{t-i}^{2} X_{t-j}^{2} X_{t}^{2}}{2 h_{t}^{3}}+\frac{X_{t-i}^{2} X_{t-j}^{2}}{2 h_{t}^{2}}\right\} \\
& =E\left\{E\left\{\frac{2 X_{t-i}^{2} X_{t-j}^{2} X_{t}^{2}}{2 h_{t}^{3}} \mid \mathscr{F}_{i-1}\right\}+\frac{X_{t-i}^{2} X_{t-j}^{2}}{2 h_{t}^{2}}\right\} \\
& =E\left\{\frac{2 X_{t-i}^{2} X_{t-j}^{2}}{2 h_{t}^{2}}+\frac{X_{t-i}^{2} X_{t-j}^{2}}{2 h_{t}^{2}}\right\} \\
& =E\left\{\frac{3 X_{t-i}^{2} X_{t-j}^{2}}{2 h_{t}^{2}}\right\} \\
& =E\left\{\frac{3 X_{t-i}^{2} X_{t-j}^{2}}{2\left(\alpha_{0}+\alpha_{1} X_{i-1}^{2}+\cdots+\alpha_{r} X_{i-r}^{2}\right)\left(\alpha_{0}+\alpha_{1} X_{i-1}^{2}+\cdots+\alpha_{r} X_{i-r}^{2}\right)}\right\} \\
& \leq E\left\{\frac{3 X_{t-i}^{2} X_{t-j}^{2}}{2 \alpha_{1}^{i} X_{t-i}^{2}\left(\prod_{p=1}^{i-1} \varepsilon_{t-p}^{2}\right) \alpha_{1}^{j} X_{t-j}^{2}\left(\prod_{p=1}^{j-1} \varepsilon_{t-p}^{2}\right)}\right\} \\
& \leq E\left\{\frac{3}{2 \alpha_{1}^{i+j}\left(\prod_{p=1}^{i-1} \varepsilon_{t-p}^{2}\right)\left(\prod_{p=1}^{j-1} \varepsilon_{t-p}^{2}\right)}\right\} \\
& <\infty .
\end{aligned}
$$

O caso $j=0$ ou $i=0$ é análogo. Usando (2.26), e aplicando os Teoremas 2.26 e 2.5, 
temos que

$$
\lim _{n \rightarrow \infty} \frac{\frac{\partial^{2} \log L_{n, k}\left(\theta_{r}\right)}{\partial \alpha_{i} \alpha_{j}}}{n}=-m(i, j) \quad \text { q.c. }
$$

De Francq \& Zakoian (2010) (eq. 7.89, pg. 179) temos que

$$
\lim _{n \rightarrow \infty}\left|\frac{\frac{\partial^{2} \log L_{n, k}\left(\theta_{r}\right)}{\partial \alpha_{i} \alpha_{j}}}{n}-\frac{\frac{\partial^{2} \log L_{n, k}(\dot{\theta})}{\partial \alpha_{i} \alpha_{j}}}{n}\right|=0 \quad \text { q.c. }
$$

e portanto

$$
\lim _{n \rightarrow \infty} \frac{\frac{\partial^{2} \log L_{n, k}(\dot{\theta})}{\partial \alpha_{i} \alpha_{j}}}{n}=-m(i, j) \quad \text { q.c. }
$$

(ii) Para $\theta_{k}=\left(a_{0}, \ldots, a_{k}\right) \in \mathbb{R}^{k+1}$ qualquer, tal que $\left\|\theta_{k}\right\|>0$, temos

$$
\begin{aligned}
\theta_{k} A_{2} \theta_{k}^{T} & =\theta_{k} E\left\{-D_{\theta}^{2}\left(\log L_{n, k}\left(\theta_{r}\right)\right)\right\} \theta_{k}^{T} \\
& =E\left\{\frac{1}{2 h_{t}^{2}}\left[\begin{array}{lll}
a_{0} & \ldots & a_{k}
\end{array}\right]\left[\begin{array}{cccc}
1 & X_{t-1}^{2} & \ldots & X_{t-k}^{2} \\
X_{t-1}^{2} & X_{t-1}^{2} X_{t-1}^{2} & \ldots & X_{t-k}^{2} X_{t-1}^{2} \\
\vdots & \vdots & \ddots & \vdots \\
X_{t-k}^{2} & X_{t-k}^{2} X_{t-1}^{2} & \ldots & X_{t-k}^{2} X_{t-k}^{2}
\end{array}\right]\left[\begin{array}{c}
a_{0} \\
\vdots \\
a_{k}
\end{array}\right]\right\} \\
& =E\left\{\frac{\left(a_{0}+a_{1} X_{t-1}^{2}+\cdots+a_{k} X_{t-k}^{2}\right)^{2}}{2 h_{t}^{2}}\right\} \\
& =E\left\{\frac{h_{t}^{2}\left(\theta_{k}\right)}{2 h_{t}^{2}\left(\theta_{r}\right)}\right\} \\
& >0 .
\end{aligned}
$$

(iii) Esse é um caso particular da equação B3 de Comte \& Lieberman (2003).

Como, pelo Teorema 2.26, os modelos ARCH são inseridos no contexto de cadeias de Markov, seria natural a utilização da Lei do Logaritmo Iterado apresentada no Teorema 2.5. Entretanto, as Condições 2.1 ou 2.2 não estão estabelecidas de forma suficiente para processos ARCH. Dessa forma, optou-se nesse trabalho por utilizar o Teorema 2.6 na demonstração do próximo Lema. 
Lema 2.28. Seja $\left\{X_{t}\right\}_{t \in \mathbb{N}}$ um ARCH(r) satisfazendo as Condições 2.5 e 2.6, $k \geq r, \log L_{n, k}$ como definido em (2.24), $i \in\{0, \cdots, k\}$, então

$$
\limsup _{n \rightarrow \infty} \frac{\frac{\partial \log L_{n, k}\left(\theta_{r}\right)}{\partial \alpha_{i}}}{\sqrt{2 n \log \log n}}=E\left(\frac{X_{t-i}^{4}}{2 h_{t}^{2}}\right)^{1 / 2}<\infty q . c .,
$$

e

$$
\liminf _{n \rightarrow \infty} \frac{\frac{\partial \log L_{n, k}\left(\theta_{r}\right)}{\partial \alpha_{i}}}{\sqrt{2 n \log \log n}}=-E\left(\frac{X_{t-i}^{4}}{2 h_{t}^{2}}\right)^{1 / 2}>-\infty \text { q.c. }
$$

Demonstração. Assumimos $\mathscr{F}_{t}=\sigma\left(X_{1}, \ldots, X_{t}\right), Z_{t}=t^{\delta}, \delta>1$,

$$
U_{t}=\frac{X_{t-i}^{2}}{2 h_{t}}\left(\frac{X_{t}^{2}}{h_{t}}-1\right) \quad e \quad W_{n}=\left[n E\left(\frac{X_{n-i}^{4}}{2 h_{n}^{2}}\right)\right]^{1 / 2}
$$

Para aplicar o Teorema 2.6, basta demonstrar as hipóteses (L1-L4) que seguem.

(L1) Pela desigualdade de Chebyshev temos que

$$
\begin{aligned}
P\left(\left|U_{t}\right|>Z_{t}\right) & =P\left(\left|\frac{X_{t-i}^{2}}{2 h_{t}}\left(\frac{X_{t}^{2}}{h_{t}}-1\right)\right|>t^{\delta}\right) \\
& \leq P\left(\left|\frac{1}{\alpha_{i}}\left(\frac{X_{t}^{2}}{h_{t}}-1\right)\right|>t^{\delta}\right) \\
& =P\left(\left|\left(\frac{X_{t}^{2}}{h_{t}}-1\right)\right|>\alpha_{i} t^{\delta}\right) \\
& \leq \frac{1}{\alpha_{i}^{2} t^{2 \delta}} E\left[\left(\varepsilon_{t}^{2}-1\right)^{2}\right] \\
& =\frac{1}{\alpha_{i}^{2} t^{2 \delta}} E\left[\varepsilon_{t}^{4}-2 \varepsilon_{t}^{2}+1\right] \\
& =\frac{2}{\alpha_{i}^{2} t^{2 \delta}} .
\end{aligned}
$$

Então temos que

$$
\sum_{t=1}^{\infty} P\left(\left|U_{t}\right|>Z_{t}\right) \leq \sum_{t=1}^{\infty} \frac{2}{\alpha_{i}^{2} t^{2 \delta}}<\infty
$$

e usando o Lema de Borel-Cantelli temos que

$$
P\left(\left\{\omega: \mathbb{I}\left(\left|U_{t}\right|>t^{\delta}\right)=1 \text { infinitas vezes }\right\}\right)=P\left(\left\{\omega:\left|U_{t}\right|>t^{\delta} \text { infinitas vezes }\right\}\right)=0
$$


e portanto

$$
\lim _{n \rightarrow \infty} \frac{\sum_{t=1}^{n} U_{t} \mathbb{I}\left(\left|U_{t}\right|>Z_{t}\right)-E\left[U_{t} \mathbb{I}\left(\left|U_{t}\right|>Z_{t}\right) \mid \mathscr{F}_{t-1}\right]}{\sqrt{2 W_{n}^{2} \log \log W_{n}^{2}}}=0 \quad \text { q.c. }
$$

(L2)

$$
\begin{aligned}
E\left(U_{t} \mid \mathscr{F}_{t-1}\right) & =\frac{X_{t-i}^{2}}{2 h_{t}} E\left[\left(\frac{X_{t}^{2}}{h_{t}}-1\right) \mid \mathscr{F}_{t-1}\right] \\
& =0 e \\
E\left[E\left(U_{t}^{2} \mid \mathscr{F}_{t-1}\right)\right] & =E\left[\frac{X_{t-i}^{4}}{4 h_{t}^{2}}\left(\varepsilon_{t}^{2}-1\right)^{2}\right] \\
& =E\left[\frac{X_{t-i}^{4}}{4 h_{t}^{2}}\right] E\left[\varepsilon_{t}^{4}-2 \varepsilon_{t}^{2}+1\right] \\
& =E\left(\frac{X_{t-i}^{4}}{2 h_{t}^{2}}\right) \leq E\left(\frac{1}{2 \alpha_{i}}\right)<\infty
\end{aligned}
$$

e portanto, usando o item (i) do Teorema 2.5 (LFGN), temos que

$$
\lim _{n \rightarrow \infty} \frac{\sum_{t=1}^{n} E\left[U_{t}^{2} \mid \mathscr{F}_{t-1}\right]}{n E\left(\frac{X_{1-i}^{4}}{2 h_{1}^{2}}\right)}=1 \quad \text { q.c. }
$$

Usando o Teorema da Convergência Dominada, temos que

$$
\lim _{t \rightarrow \infty} E\left[U_{t} \mathbb{I}\left(\left|U_{t}\right| \leq t^{\delta}\right) \mid \mathscr{F}_{t-1}\right]=0 \quad \text { q.c. }
$$

Agora, considerando $\varepsilon>0$ arbitrário, é necessário encontrar uma cota superior somável em $t$ para

$$
P\left[\left|E\left(U_{t}^{2} \mid \mathscr{F}_{t-1}\right)-E\left(U_{t}^{2} I\left(\left|U_{t}\right| \leq t^{\delta}\right) \mid \mathscr{F}_{t-1}\right)\right|>\varepsilon\right]
$$

e aplicar Borel-Cantelli para obter

$$
\lim _{t \rightarrow \infty}\left[E\left(U_{t}^{2} \mathbb{I}\left(\left|U_{t}\right| \leq t^{\delta}\right) \mid \mathscr{F}_{t-1}\right)-E\left(U_{t}^{2} \mid \mathscr{F}_{t-1}\right)\right]=0 \quad \text { q.c. }
$$


e aplicar o Teorema Médio de Cesàro (Apêndice A) para concluir

$$
\begin{aligned}
\lim _{n \rightarrow \infty} \frac{\sum_{t=1}^{n} E\left[U_{t}^{2} \mathbb{I}\left(\left|U_{t}\right| \leq Z_{t}\right) \mid \mathscr{F}_{t-1}\right]-E\left[U_{t} \mathbb{I}\left(\left|U_{t}\right| \leq Z_{t}\right) \mid \mathscr{F}_{t-1}\right]^{2}}{W_{n}^{2}} & =\lim _{n \rightarrow \infty} \frac{\sum_{t=1}^{n} E\left[U_{t}^{2} \mid \mathscr{F}_{t-1}\right]}{n E\left(\frac{X_{1-i}^{4}}{2 h_{1}^{2}}\right)} \\
& =1 \text { q.c. }
\end{aligned}
$$

Usando a desigualdade generalizada de Chebyshev (Apêndice B)

$$
\begin{aligned}
P\left[\left|E\left(U_{t}^{2} \mid \mathscr{F}_{t-1}\right)-E\left(U_{t}^{2} I\left(\left|U_{t}\right| \leq t^{\delta}\right) \mid \mathscr{F}_{t-1}\right)\right|>\varepsilon\right] \\
\leq \frac{1}{\varepsilon} E\left[\left|E\left(U_{t}^{2} \mid \mathscr{F}_{t-1}\right)-E\left(U_{t}^{2} I\left(\left|U_{t}\right| \leq t^{\delta}\right) \mid \mathscr{F}_{t-1}\right)\right|\right]
\end{aligned}
$$

$\mathrm{e}$

$$
\begin{aligned}
E\left[\left|E\left(U_{t}^{2} \mid \mathscr{F}_{t-1}\right)-E\left(U_{t}^{2} I\left(\left|U_{t}\right| \leq t^{\delta}\right) \mid \mathscr{F}_{t-1}\right)\right|\right] & =E\left[\left|E\left(U_{t}^{2} \mid \mathscr{F}_{t-1}\right)-E\left(U_{t}^{2} I\left(\left|U_{t}\right| \leq t^{\delta}\right) \mid \mathscr{F}_{t-1}\right) \pm E\left(U_{t}^{2} I\left(\left|U_{t}\right|>t^{\delta}\right) \mid \mathscr{F}_{t-1}\right)\right|\right] \\
& =E\left[E\left(U_{t}^{2} I\left(\left|U_{t}\right|>t^{\delta}\right) \mid \mathscr{F}_{t-1}\right)\right] \\
& =E\left[U_{t}^{2} I\left(\left|U_{t}\right|>t^{\delta}\right)\right] \\
& \leq E\left[U_{t}^{4}\right]^{1 / 2} E\left[I\left(\left|U_{t}\right|>t^{\delta}\right)\right]^{1 / 2} \\
& =E\left[U_{t}^{4}\right]^{1 / 2} \mathrm{P}\left(\left|U_{t}\right|>t^{\delta}\right)^{1 / 2}
\end{aligned}
$$

Usando (2.30) e (2.31) para $c>0$ apropriado, obtemos

$$
\begin{aligned}
E\left[\left|E\left(U_{t}^{2} \mid \mathscr{F}_{t-1}\right)-E\left(U_{t}^{2} I\left(\left|U_{t}\right| \leq t^{\delta}\right) \mid \mathscr{F}_{t-1}\right)\right|\right] & \leq c\left(\frac{2}{\alpha_{i}^{2} t^{2 \delta}}\right)^{1 / 2} \\
& =c \frac{2^{1 / 2}}{\alpha_{i} t^{\delta}}
\end{aligned}
$$

que é somável em $t$. 
(L3) Temos que

$$
\begin{aligned}
0 & \leq E\left[U_{t}^{4} \mathbb{I}\left(\left|U_{t}\right| \leq Z_{t}\right) \mid \mathscr{F}_{t-1}\right] \\
& \leq E\left[U_{t}^{4} \mid \mathscr{F}_{t-1}\right] \\
& =E\left[\left(\frac{X_{t-i}^{2}}{2 h_{t}}\right)^{4}\left(\frac{X_{t}^{2}}{h_{t}}-1\right)^{4} \mid \mathscr{F}_{t-1}\right] \\
& \leq \frac{1}{\alpha_{i}^{4}} E\left[\left(\varepsilon_{t}^{2}-1\right)^{4} \mid \mathscr{F}_{t-1}\right] \\
& =c_{1} E\left[\left(\varepsilon_{t}^{2}-1\right)^{4} \mid \mathscr{F}_{t-1}\right]
\end{aligned}
$$

para $\delta_{1} \in\left(\frac{1}{2}, 1\right)$, usando as desigualdades de Chebyshev e de Jensen,

$$
\begin{aligned}
\mathrm{P}\left[c_{1} E\left[\left(\varepsilon_{t}^{2}-1\right)^{4} \mid \mathscr{F}_{t-1}\right]>t^{\delta_{1}}\right] & \leq \frac{c_{1}^{2}}{t^{2 \delta_{1}}} E\left[E\left[\left(\varepsilon_{t}^{2}-1\right)^{4} \mid \mathscr{F}_{t-1}\right]^{2}\right] \\
& \leq \frac{c_{1}^{2}}{t^{2 \delta_{1}}} E\left[\left(\varepsilon_{t}^{2}-1\right)^{8}\right] \\
& \leq c_{2} \frac{1}{t^{2 \delta_{1}}}
\end{aligned}
$$

e portanto,

$$
\sum_{t=1}^{\infty} \mathrm{P}\left[E\left[U_{t}^{4} \mathbb{I}\left(\left|U_{t}\right| \leq Z_{t}\right) \mid \mathscr{F}_{t-1}\right]>t^{\delta_{1}}\right] \leq \sum_{t=1}^{\infty} \frac{c_{2}}{t^{2 \delta_{1}}}<\infty .
$$

Usando o Lema de Borel-Cantelli,

$$
\mathrm{P}\left[E\left[U_{t}^{4} \mathbb{I}\left(\left|U_{t}\right| \leq Z_{t}\right) \mid \mathscr{F}_{t-1}\right]>t^{\delta_{1}} \quad \text { i.o. }\right]=0
$$

e logo

$$
\lim _{n \rightarrow \infty} \sum_{t=1}^{n} \frac{E\left[U_{t}^{4} \mathbb{I}\left(\left|U_{t}\right| \leq Z_{t}\right) \mid \mathscr{F}_{t-1}\right]}{W_{t}^{4}} \leq \lim _{n \rightarrow \infty} \sum_{t=1}^{n} \frac{1}{t^{2-\delta_{1}}}<\infty \quad \text { q.c. }
$$

(L4) Como $E\left(X_{t}^{4}\right)>0$, caso contrário $X_{t} \equiv 0$ q.c., então, usando a estacionaridade, temos

$$
\lim _{n \rightarrow \infty} \frac{W_{n}}{W_{n+1}}=\lim _{n \rightarrow \infty} \frac{\left[n E\left(X_{1-i}^{4} / h_{1}^{2}\right)\right]^{1 / 2}}{\left[(n+1) E\left(X_{1-i}^{4} / h_{1}^{2}\right)\right]^{1 / 2}}=1 \quad \text { q.c. } e
$$




$$
\lim _{n \rightarrow \infty} W_{n}=E\left(X_{1-i}^{4} / h_{1}^{2}\right)^{1 / 2} \lim _{n \rightarrow \infty} \sqrt{n}=\infty .
$$

Ressalta-se que a desigualdade generalizada de Chebyshev e o Teorema Médio de Cesàro foram utilizados por Hafner \& Preminger (2009a), em situação semelhante, para demonstrar a normalidade assintótica do estimador do parâmetro $\theta_{r}$ no caso de processos FACTORGARCH.

Corolário 2.29. Seja $\left\{X_{t}\right\}_{t \in \mathbb{N}}$ um ARCH(r), satisfazendo as Condições 2.5 e 2.6, $k \geq r$, $\log L_{n, k}$ como definido em (2.24), então existe $c_{5} \in(0, \infty)$ tal que

$$
\limsup _{n \rightarrow \infty} \frac{\left\|D_{\theta}^{1} \log L_{n, k}\left(\theta_{r}\right)\right\|}{\sqrt{2 \log \log n}} \leq c_{5} \quad \text { q.c. }
$$

Demonstração.

$$
\begin{aligned}
\limsup _{n \rightarrow \infty} \frac{\left\|D_{\theta}^{1} \log L_{n, k}\left(\theta_{r}\right)\right\|}{\sqrt{2 \log \log n}} & \leq \limsup _{n \rightarrow \infty} \sum_{i=0}^{k} \frac{\left|\frac{\partial \log L_{n, k}\left(\theta_{r}\right)}{\partial \alpha_{i}}\right|}{\sqrt{2 n \log \log n}} \\
& \leq \sum_{i=0}^{k} \limsup _{n \rightarrow \infty} \frac{\left|\frac{\partial \log L_{n, k}\left(\theta_{r}\right)}{\partial \alpha_{i}}\right|}{\sqrt{2 n \log \log n}} \\
& \leq \sum_{i=0}^{k} E\left(\frac{X_{t-i}^{4}}{2 h_{t}^{2}}\right)^{1 / 2} \\
& =c_{5} \text { q.c. }
\end{aligned}
$$

O próximo Lema contém resultados técnicos que são utilizados no que segue.

Lema 2.30. Seja $\mathbb{X}$ um ARCH(r) satisfazendo as Condições 2.5 e 2.6, $k \geq 0, A \subseteq \Theta_{k}$, então

(i) $\operatorname{Se} \bar{\theta}=\left(\alpha_{0}, \ldots, \alpha_{k}\right) \in A$

$$
\begin{gathered}
E\left|h_{t}(\bar{\theta})\right|<\infty, \\
E\left|\log \left(h_{t}(\bar{\theta})\right)\right|<\infty \quad e \\
E\left|\log \left(\frac{1}{2 \pi h_{t}(\bar{\theta})} e^{-\frac{X_{t}^{2}}{2 h_{t}(\bar{\theta})}}\right)\right|<\infty .
\end{gathered}
$$


(ii) Para $i \in\{0, \ldots, k\}$,

$$
E \sup _{\theta \in A}\left|\frac{X_{t-i}^{2}}{2 h_{t}(\theta)}\left(\frac{X_{t}^{2}}{h_{t}(\theta)}-1\right)\right|<\infty .
$$

Demonstração. (i) Como $E\left(X_{t}^{2}\right)<\infty$,

$$
\begin{aligned}
E\left|h_{t}(\bar{\theta})\right| & \leq E\left|\alpha_{0}+\sum_{i=1}^{k} \alpha_{i} X_{t-i}^{2}\right| \\
& \leq \alpha_{0}+\sum_{i=1}^{k} \alpha_{i} E\left(X_{t-i}^{2}\right)<\infty
\end{aligned}
$$

Temos também que

$$
E\left(\log \left(h_{t}(\bar{\theta})\right)\right) \leq E\left(h_{t}\right)<\infty .
$$

Além disso, usando $c$ como na Condição 2.6, temos

$$
E\left(\log \left(h_{t}(\bar{\theta})\right)\right)^{-} \leq \max \{-\log (c), 0\}<\infty .
$$

Para a última inequação temos

$$
E\left|\log \left(\frac{1}{2 \pi h_{t}(\bar{\theta})} e^{-\frac{X_{t}^{2}}{2 h_{t}(\bar{\theta})}}\right)\right| \leq c_{1}+\frac{1}{c} E\left|X_{t}^{2}\right|+E\left|\log \left(h_{t}\right)\right|<\infty .
$$

(ii)

$$
\begin{aligned}
E \sup _{\theta \in A}\left|\frac{X_{t-i}^{2}}{2 h_{t}(\theta)}\left(\frac{X_{t}^{2}}{h_{t}(\theta)}-1\right)\right| & \leq E\left|\frac{X_{t-i}^{2}}{c} \frac{X_{t}^{2}}{c}\right| \\
& \leq E\left|\frac{X_{t-i}^{4}}{c}\right|^{1 / 2} E\left|\frac{X_{t}^{4}}{c}\right|^{1 / 2}<\infty .
\end{aligned}
$$

Abaixo demonstramos $\mathrm{H} 1$ para processos $\mathrm{ARCH}$. O resultado análogo demonstrado para processos AR utilizou um argumento simples, fundamentado na expansão de Taylor e considerando que as derivadas de terceira ordem de $\log L_{n, k}$ são nulas nos processos $\mathrm{AR}$, o que não ocorre no geral. Assim, optou-se por desenvolver uma demonstração (abaixo) utilizando 
a comparação das densidades pela divergência de Kullback-Leibler em um contexto mais geral. Para isso foi necessário atentar para alguns detalhes de convergência de sequências.

Teorema 2.31. Seja $\mathbb{X}$ um ARCH(r) satisfazendo as Condições 2.5 e 2.6, $k<r$, então

$$
\lim _{n \rightarrow \infty} \frac{\log L_{n}\left(\hat{\theta}_{r}\right)-\log L_{n}\left(\hat{\theta}_{k}\right)}{n}>0 \quad \text { q.c. }
$$

Demonstração. Basta mostrar o resultado considerando

$$
\log L_{n, k}(\theta)=\sum_{t=1+k}^{n} l_{t}(\theta)
$$

e

$$
l_{t}(\theta)=\log (f(\theta))=\log \left(\frac{1}{\sqrt{2 \pi h_{t}}} e^{-\frac{X_{t}^{2}}{2 h_{t}}}\right)
$$

em (2.23). Usando o Lema 2.30, temos que

$$
E\left[\left|l_{t}(\theta)\right|\right]<\infty
$$

e portanto, usando o Teorema 2.5,

$$
\lim _{n \rightarrow \infty} \frac{\log L_{n}\left(\theta_{r}\right)}{n}=\lim _{n \rightarrow \infty} \frac{\sum_{t=1+k}^{n} l_{t}\left(\theta_{r}\right)}{n}=E\left(l_{1}\left(\theta_{r}\right)\right)=c_{1}<\infty \quad \text { q.c. }
$$

Usando o Teorema do valor médio, para $\dot{\theta}=s \theta_{r}+(1-s) \hat{\theta}_{r}, s \in(0,1), n$ suficientemente grande e $B_{\delta}\left(\theta_{r}\right)$ uma vizinhança suficientemente pequena de $\theta_{r}$, temos

$$
\begin{aligned}
\left|\frac{\sum_{t=1+r}^{n} l_{t}\left(\hat{\theta}_{r}\right)}{n}-\frac{\sum_{t=1+r}^{n} l_{t}\left(\theta_{r}\right)}{n}\right| & =\left|\frac{\sum_{t=1+r}^{n} D_{\theta}^{1} l_{t}(\dot{\theta})}{n}\left(\hat{\theta}_{r}-\theta_{r}\right)\right| \\
& \leq \sup _{\theta \in B_{\delta}\left(\theta_{r}\right)}\left\|\frac{\sum_{t=1+r}^{n} D_{\theta}^{1} l_{t}(\dot{\theta})}{n}\right\|\left\|\left(\hat{\theta}_{r}-\theta_{r}\right)\right\|
\end{aligned}
$$

Aplicando o Teorema 2.5, usando o item (ii) do Lema 2.30 e a consistência forte de $\hat{\theta}_{r}$, 


$$
\left|\frac{\sum_{t=1+r}^{n} l_{t}\left(\hat{\theta}_{r}\right)}{n}-\frac{\sum_{t=1+r}^{n} l_{t}\left(\theta_{r}\right)}{n}\right| \underset{q . c .}{\rightarrow} 0 .
$$

Por outro lado, temos que, como o estimador é de máxima verossimilhança e $\Theta_{k} \subset \Theta_{r}$,

$$
\begin{aligned}
\lim _{n \rightarrow \infty} \frac{\log L_{n}\left(\hat{\theta}_{k}\right)}{n} & \leq \lim _{n \rightarrow \infty} \frac{\log L_{n}\left(\hat{\theta}_{r}\right)}{n} \\
& =c_{1} \text { q.c. }
\end{aligned}
$$

e portanto,

$$
\lim _{n \rightarrow \infty} \frac{\log L_{n}\left(\hat{\theta}_{k}\right)}{n} \leq \limsup _{n \rightarrow \infty} \frac{\sum_{t=1+k}^{n} l_{t}\left(\hat{\theta}_{k}\right)}{n}=c_{2} \leq c_{1} \quad \text { q.c. }
$$

Seja $n_{i}$ uma subsequência de $n$ tal que

$$
\lim _{n_{i} \rightarrow \infty} \frac{\sum_{t=1+k}^{n_{i}} l_{t}\left(\hat{\theta}_{k}\right)}{n_{i}}=c_{2} \text { q.c. }
$$

Usando que $\bar{\Theta}_{k}$ é compacto, tome $n_{j}$ uma subsequência de $n_{i}$ tal que $\hat{\theta}_{k}\left(n_{j}\right) \rightarrow \bar{\theta}_{k} \in \bar{\Theta}_{k}$ q.c. Temos assim que

$$
\begin{aligned}
\lim _{n \rightarrow \infty} \frac{\log L_{n}\left(\hat{\theta}_{k}\right)}{n} & \leq \limsup _{n \rightarrow \infty} \frac{\log L_{n}\left(\hat{\theta}_{k}\right)}{n} \\
& =\lim _{n_{j} \rightarrow \infty} \frac{\log L_{n_{j}}\left(\hat{\theta}_{k}\left(n_{j}\right)\right)}{n_{j}} \text { q.c. }
\end{aligned}
$$

Aplicando o mesmo raciocínio usado em (2.32), concluímos

$$
\begin{gathered}
\lim _{n_{j} \rightarrow \infty} \frac{\log L_{n_{j}}\left(\bar{\theta}_{k}\right)}{n_{j}}=E\left(l_{1}(\bar{\theta})\right) \quad e \\
\left|\frac{\sum_{t=1+k}^{n} l_{t}\left(\hat{\theta}_{k}\right)}{n}-\frac{\sum_{t=1+k}^{n} l_{t}(\bar{\theta})}{n}\right| \underset{q . c .}{\rightarrow} 0 .
\end{gathered}
$$


Além disso,

$$
\lim _{n \rightarrow \infty}\left[\frac{\log L_{n}\left(\hat{\theta}_{r}\right)}{n}-\frac{\log L_{n}\left(\hat{\theta}_{k}\right)}{n}\right] \geq E\left[\log \left(\frac{f\left(\bar{\theta}_{k}\right)}{f\left(\theta_{r}\right)}\right)\right]
$$

Por outro lado, $E\left[\log \left(\frac{f\left(\bar{\theta}_{k}\right)}{f\left(\theta_{r}\right)}\right)\right]$ é a divergência de Kullback-Leibler, que é positiva se $f\left(\bar{\theta}_{k}\right) \neq$ $f\left(\theta_{r}\right)$, e como $\theta_{r} \notin \bar{\Theta}_{k} \subseteq \mathbb{R}^{\gamma(k)}$, temos que $\theta_{r} \neq \bar{\theta}_{k}$ e então $f\left(\bar{\theta}_{k}\right) \neq f\left(\theta_{r}\right)$. Onde concluímos

$$
\lim _{n \rightarrow \infty}\left[\frac{\log L_{n}\left(\hat{\theta}_{r}\right)}{n}-\frac{\log L_{n}\left(\hat{\theta}_{k}\right)}{n}\right] \geq E\left[\log \left(\frac{f\left(\bar{\theta}_{k}\right)}{f\left(\theta_{r}\right)}\right)\right]>0 .
$$

Teorema 2.32. Seja $\mathbb{X}$ um ARCH(r) satisfazendo as Condições 2.5 e 2.6 e $\hat{r}_{\text {edc }}$ como na Definição 2.24. Então, $\hat{r}_{\text {edc }}$ é fortemente consistente $\left(\hat{r}_{\text {edc }} \underset{q . c .}{\rightarrow} r\right)$ se

$$
\lim _{n \rightarrow \infty} \frac{c_{n}}{n}=0 \quad e \quad \liminf _{n \rightarrow \infty} \frac{c_{n}}{\log \log n}=\infty
$$

Demonstração. O conjunto dos possíveis valores de $\theta_{r}, \Theta_{r}$, definido em (2.22), é aberto e $\theta_{r} \in \Theta_{r}$. De Weiss (1986), temos que o estimador de máxima verossimilhança $\hat{\theta}_{k}$ é fortemente consistente para $k \geq r$, isto é, vale (1.10). As derivadas de $\log L_{n, k},(2.25)$ e (2.26) são contínuas em relação a $\theta$ e a $x_{1}^{n}$ e portanto são mensuráveis em relação a $x_{1}^{n}$. Temos de (2.27) que (1.11) é satisfeita. Usando o Teorema 2.27 e o Corolário 2.29 temos as condições satisfeitas para a aplicação do item (ii) do Teorema 1.6 e com isso temos (H2) satisfeita. A hipótese (H1) é segue do Teorema 2.31. O resultado segue do item (i) do Teorema 1.5.

Corolário 2.33. Seja $\mathbb{X}$ um ARCH(r) satisfazendo as Condições 2.5 e 2.6, $K \geq r$. Então o estimador $\hat{r}_{b i c}$, como definido abaixo, é fortemente consistente.

$$
\hat{r}_{b i c}=\underset{k \in\{0, \ldots, K\}}{\operatorname{argmin}}\left\{-\log L_{n, k}\left(\hat{\theta}_{k}\right)+\frac{(k+1)}{2} \log n\right\}
$$

para $\log L_{n, k}\left(\hat{\theta}_{k}\right)$ como definida em (2.24).

Demonstração. $c_{n}=\log n / 2$ e satisfaz (2.33). 


\subsection{Modelo ARCH multivariado generalizado (BEKK-GARCH)}

Desde sua criação por Engle (1982), o modelo ARCH teve diversas generalizações e adaptações em que se destacam os modelos GARCH (Bollerslev 1986), NGARCH (Engle \& $\mathrm{Ng}$ 1993), EGARCH (Nelson 1991) e generalizações multivariadas como BEKK-GARCH (Engle \& Kroner 1995), CCC-GARCH (Bollerslev 1990), VEC-GARCH (Bollerslev, Engle $\&$ Wooldridge 1988), dentre outros.

Os modelos multivariados tem especial aplicação na seleção de portfólios e precificação de ativos (Hafner \& Preminger 2009b). Na classe dos modelos multivariados, o BEKKGARCH se destaca por ser geral e por haver avanços significativos disponíveis na literatura. Dos citados, apenas o VEC-GARCH é mais geral que o modelo BEKK-GARCH e, mesmo assim, os casos VEC-GARCH que não são representáveis na modelagem BEKK-GARCH são de certa forma degenerados (Stelzer 2008).

Boussama (1998), utilizando técnicas de geometria algébrica, inseriu os modelos BEKKGARCH no contexto de cadeias de Markov e demonstrou a ergodicidade geométrica desses modelos a partir de determinadas condições. Com algumas alterações, esses resultados também foram publicados por Boussama, Fuchs \& Stelzer (2011).

Comte \& Lieberman (2003) utilizou os resultados de Boussama (1998) para demonstrar as condições propostas por Jeantheau (1998) para obter a consistência forte de estimadores de máxima verossimilhança para processos BEKK-GARCH. Usando as condições de Basawa \& Heyde (1976), a normalidade assintótica do estimador de máxima verossimilhança também é estabelecida por Comte \& Lieberman.

Assim como em processos ARCH, não há a formalização de estimadores de ordem para modelos BEKK-GARCH, embora os critérios de informação AIC e BIC vêm sendo utilizados (Francq \& Zakoian 2010).

Nessa Seção, a classe de estimadores EDC é definida para modelos BEKK-GARCH e são demonstradas a consistência forte para uma subclasse, que inclui o estimador de ordem baseado no critério de informação BIC. A técnica utilizada é semelhante à utilizada para o caso de modelos $\mathrm{ARCH}$, a maior diferença está no uso de cálculo matricial, que é necessário 
para a manipulação dos objetos existentes na definição multivariada.

\subsubsection{Definições}

No que segue é apresentada a definição de modelo BEKK-GARCH e da notação utilizada nos próximos resultados.

Definição 2.34. Uma sequência de variáveis aleatórias $\mathbb{X}=\left\{X_{t}\right\}_{t \in \mathbb{N}}$ com valores em $\mathbb{R}^{m}$ é um processo Autoregressivo de Heteroscedasticidade Condicional Generalizado na modelagem BEKK (BEKK-GARCH) de ordem $(p, q) \in \mathbb{N}^{2}$, denotado por BEKK-GARCH $(p, q)$, se satisfaz as condições abaixo.

(i) Para todo $t \in \mathbb{N}$,

$$
X_{t}=\left(H_{t}\right)^{\frac{1}{2}} \varepsilon_{t} .
$$

(ii) Para $C, A_{l s}$ e $B_{l s}$ matrizes $(m \times m)$, C positiva definida e $N \in \mathbb{N}$,

$$
H_{t}=C+\sum_{l=1}^{q}\left(\sum_{s=1}^{N} A_{l s} X_{t-l} X_{t-l}^{\prime} A_{l s}^{\prime}\right)+\sum_{l=1}^{p}\left(\sum_{s=1}^{N} B_{l s} H_{t-l} B_{l s}^{\prime}\right) .
$$

(iii) $\left\{\varepsilon_{t}\right\}_{t \in \mathbb{N}}$ é uma sequência de variáveis aleatórias i.i.d em $\mathbb{R}^{m}$ com distribuição $\mathscr{N}\left(0, I_{m}\right)$, para $I_{m}$ a matriz identidade $(m \times m)$.

Definição 2.35. Seja $A=\left(a_{i j}\right) \in \mathcal{M}$ uma matriz e $\mathcal{M}$ o espaço das matrizes $(m \times m)$.

(i) O operador vec $: \mathcal{M} \rightarrow \mathbb{R}^{m \times m}$ é definido por

$$
\operatorname{vec}(A)=\left(a_{11}, a_{21}, \ldots, a_{m 1}, a_{12}, \ldots, a_{m 2}, \ldots, a_{1 m}, \ldots, a_{m m}\right)^{\prime}
$$

(ie., empilha as colunas de A).

(ii) O operador vech: $\mathcal{M} \rightarrow \mathbb{R}^{m(m+1) / 2}$ é definido por

$$
\operatorname{vech}(A)=\left(a_{11}, a_{21}, \ldots, a_{m 1}, a_{22}, \ldots, a_{m 2}, \ldots, a_{m m}\right)^{\prime}
$$

(ie., empilha as colunas da parte triangular inferior de A). 
(iii) $D_{m}=\left(d_{i j}\right)$ é a matriz duplicação, em que

$$
\begin{gathered}
d_{i j}=\mathbb{I}\left[(i, j) \in A_{d}\right] \text { e } \\
A_{d}=\left\{(a, b) \in \mathbb{N}^{2}:(a, b)=((j-1) m+i,(j-1)(m-j / 2)+i)\right. \text { ou } \\
(a, b)=((i-1) m+j,(j-1)(m-j / 2)+i) \\
i, j \in \mathbb{N} \text { e } i \geq j\} .
\end{gathered}
$$

(iv) $D_{m}^{+}=\left(D_{m}^{\prime} D_{m}\right)^{-1} D_{m}^{\prime}$.

(v) Se B é matriz $(m \times n)$ e C é matriz $\left(m^{\prime} \times n^{\prime}\right)$, então o Produto de Kronecker $B \otimes C$ é a matriz $\left(\mathrm{mm}^{\prime} \times n n^{\prime}\right)$ com os blocos $a_{i j} B$.

(vi) O raio espectral de A é definido por $\rho(A)=\max \left\{\left|\lambda_{i}\right|: \lambda_{i}\right.$ é autovalor de $\left.A\right\}$.

(vii) $\|A\|$ é a norma de Frobenius, isto é,

$$
\|A\|^{2}=\sum_{i, j} a_{i j}^{2}
$$

Usando a notação definida, temos o seguinte resultado que pode ser encontrado em Francq \& Zakoian (2010).

Proposição 2.36 (Francq \& Zakoian (2010)). Seja A matriz $(m \times m)$, B e C matrizes tais que o produto ABC esteja bem definido. Então

$$
\begin{gathered}
\operatorname{vec}(A)=D_{m} \operatorname{vech}(A), \\
\operatorname{vech}(A)=D_{m}^{+} \operatorname{vec}(A) e \\
\operatorname{vec}(A B C)=\left(C^{\prime} \otimes A\right) \operatorname{vec}(B) .
\end{gathered}
$$

A condição a seguir, necessária para a obtenção de ergodicidade de processos BEKKGARCH, foi proposta por Boussama (1998) e é utilizada por diversos trabalhos, dentre eles Comte \& Lieberman (2003). 
Condição 2.7 (Ergodicidade). Para $\mathbb{X}$ um BEKK-GARCH(p,q),

$$
\rho\left(\sum_{l=1}^{q} \tilde{A}_{l}+\sum_{l=1}^{p} \tilde{B}_{l}\right)<1
$$

para

$$
\tilde{A}_{l}=D_{m}^{+} \sum_{s=1}^{N}\left(A_{l s} \otimes A_{l s}\right) D_{m} \quad e \quad \tilde{B}_{l}=D_{m}^{+} \sum_{s=1}^{N}\left(B_{l s} \otimes B_{l s}\right) D_{m} .
$$

\section{Principais propriedades do processo BEKK-GARCH}

(a) $E\left(X_{t}\right)=\overrightarrow{0}$

$$
E\left(X_{t}\right)=E\left(E\left(X_{t} \mid \mathscr{F}_{t-1}\right)\right)=E(0)=0 .
$$

(b) $E\left(X_{t} X_{t-k}\right)=\overrightarrow{0}$, se $k \geq 1$.

$$
E\left(X_{t} X_{t-k}\right)=E\left(E\left(X_{t} X_{t-k} \mid \mathscr{F}_{i-1}\right)\right)=E\left(X_{t-k} E\left(X_{t} \mid \mathscr{F}_{i-1}\right)\right)=0
$$

(c) $E\left(X_{t} X_{t}^{\prime} \mid \mathscr{F}_{t-1}\right)=H_{t} \quad$ q.c. $($ Comte \& Lieberman 2003).

(d) Supondo $N=1$, um BEKK-GARCH $\left(k_{1}, k_{2}\right) \mathbb{X}$ pode ser imerso em uma classe de modelos parcialmente aninhados $\mathbb{M}=\left\{M_{k}\right\}_{k \in \mathbb{N}^{2}}$ tomando, $\bar{k}=\max \left\{k_{1}, k_{2}\right\}$,

$$
\Theta_{k}=\Omega_{0} \times \Omega_{1} \times \cdots \times \Omega_{2 \bar{k}}
$$

Onde $\Omega_{i}=\{0\}^{m^{2}}$ se $i / 2>k_{2}$ e $i$ é impar ou $i / 2>k_{1}$ e $i$ é par, nos demais casos $\Omega_{i} \subseteq \mathbb{R}^{m^{2}}$ com interior não vazio.

$$
\gamma(k)=m^{2}\left(1+k_{1}+k_{2}\right)
$$

assumindo $A_{i}=0$ se $i>k_{2}, B_{i}=0$ se $i>k_{2}$,

$$
\theta_{k}=\left(\operatorname{vec}(C), \operatorname{vec}\left(A_{1}\right), \operatorname{vec}\left(B_{1}\right), \ldots, \operatorname{vec}\left(A_{\bar{k}}\right), \operatorname{vec}\left(B_{\bar{k}}\right)\right) \in \Theta_{k}
$$

$\mathrm{e}$

$$
f\left(x_{1}^{n}, \theta_{k}\right)=C_{1}\left(x_{1}^{\bar{k}}\right) \prod_{t=1+\bar{k}}^{n} \frac{1}{\sqrt{(2 \pi)^{m / 2} \operatorname{det}\left(H_{t}\right)}} \exp \left(-\frac{1}{2} x_{t}^{\prime} H_{t}^{-1} x_{t}\right)
$$

para

$$
H_{t}=C+\sum_{l=1}^{k_{2}} A_{l} X_{t-l} X_{t-s}^{\prime} A_{l}^{\prime}+\sum_{l=1}^{k_{1}} B_{l} H_{t-l} B_{l}^{\prime}
$$




\section{Funções $\log L_{n, k}$ e suas derivadas}

Para uma amostra $x_{1}^{n}$, temos que a log-verossimilhança de $m_{k}=f\left(x_{1}^{n}, \theta_{k}\right), k \in \mathbb{N}^{2}$, é dada por

$$
\begin{aligned}
& \log \left(\prod_{t=1+\bar{k}}^{n} \frac{1}{\sqrt{(2 \pi)^{m / 2} \operatorname{det}\left(H_{t}\right)}} \exp \left(-\frac{1}{2} x_{t}^{\prime} H_{t}^{-1} x_{t}\right)\right)+C_{1}\left(x_{1}^{\bar{k}}\right) \\
& =\sum_{t=1+\bar{k}}^{n} \log \left(\frac{1}{\sqrt{(2 \pi)^{m / 2} \operatorname{det}\left(H_{t}\right)}} \exp \left(-\frac{1}{2} x_{t}^{\prime} H_{t}^{-1} x_{t}\right)\right)+C_{1}\left(x_{1}^{\bar{k}}\right) \\
& =\sum_{t=1+\bar{k}}^{n}\left\{-\frac{1}{2} x_{t}^{\prime} H_{t}^{-1} x_{t}-\frac{1}{2} \log \operatorname{det}\left(H_{t}\right)-\log \sqrt{(2 \pi)^{m / 2}}\right\}+C_{1}\left(x_{1}^{\bar{k}}\right) \\
& =\sum_{t=1+\bar{k}}^{n}\left\{-\frac{1}{2} x_{t}^{\prime} H_{t}^{-1} x_{t}-\frac{1}{2} \log \operatorname{det}\left(H_{t}\right)\right\}-(n-\bar{k}) \log \sqrt{(2 \pi)^{m / 2}}+C_{1}\left(x_{1}^{\bar{k}}\right)
\end{aligned}
$$

Definimos abaixo o logaritmo de $L_{n, k}$, que satisfaz a Definição 1.3.

$$
\log L_{n, k}(\theta)=\sum_{t=1+\bar{k}}^{n} l_{t}\left(\theta_{k}\right)
$$

para

$$
l_{t}\left(\theta_{k}\right)=\left\{-\frac{1}{2} x_{t}^{\prime} H_{t}^{-1} x_{t}-\frac{1}{2} \log \operatorname{det}\left(H_{t}\right)\right\} .
$$

Na literatura (exemplo em Comte \& Lieberman (2003)) a função (2.36) é assumida diretamente como a log-verossimilhança. Para $i, j \in\{1, \cdots, k\}$,

$$
\begin{aligned}
\frac{\partial \log L_{n, k}(\theta)}{\partial \alpha_{i}} & =\sum_{t=1+\bar{k}}^{n}\left\{-\frac{1}{2} x_{t}^{\prime} \frac{\partial H_{t}^{-1}}{\partial \alpha_{i}} x_{t}-\frac{1}{2 \operatorname{det}\left(H_{t}\right)}\left(\operatorname{det}\left(H_{t}\right) \operatorname{Tr}\left[H_{t}^{-1} \frac{\partial H_{t}}{\partial \alpha_{i}}\right]\right)\right\} \\
& =\sum_{t=1+\bar{k}}^{n}\left\{\frac{1}{2} x_{t}^{\prime} H_{t}^{-1} \frac{\partial H_{t}}{\partial \alpha_{i}} H_{t}^{-1} x_{t}-\frac{1}{2} \operatorname{Tr}\left[H_{t}^{-1} \frac{\partial H_{t}}{\partial \alpha_{i}}\right]\right\} \\
& =\sum_{t=1+\bar{k}}^{n} \frac{1}{2} \operatorname{Tr}\left[x_{t}^{\prime} H_{t}^{-1} \frac{\partial H_{t}}{\partial \alpha_{i}} H_{t}^{-1} x_{t}-H_{t}^{-1} \frac{\partial H_{t}}{\partial \alpha_{i}}\right] \\
& =\sum_{t=1+\bar{k}}^{n} \frac{1}{2} \operatorname{Tr}\left[x_{t} x_{t}^{\prime} H_{t}^{-1} \frac{\partial H_{t}}{\partial \alpha_{i}} H_{t}^{-1}-H_{t}^{-1} \frac{\partial H_{t}}{\partial \alpha_{i}}\right] e
\end{aligned}
$$




$$
\begin{aligned}
& \frac{\partial^{2} \log L_{n, k}(\theta)}{\partial \alpha_{i} \alpha_{j}}=\sum_{t=1+\bar{k}}^{n} \frac{1}{2} \operatorname{Tr}\left\{x_{t} x_{t}^{\prime}\left[\frac{\partial H_{t}^{-1}}{\partial \alpha_{j}} \frac{\partial H_{t}}{\partial \alpha_{i}} H_{t}^{-1}+H_{t}^{-1}\left(\frac{\partial^{2} H_{t}}{\partial \alpha_{i} \alpha_{j}} H_{t}^{-1}+\frac{\partial H_{t}}{\partial \alpha_{i}} \frac{\partial H_{t}^{-1}}{\partial \alpha_{j}}\right)\right]\right. \\
& \left.-\left(\frac{\partial H_{t}^{-1}}{\partial \alpha_{j}} \frac{\partial H_{t}}{\partial \alpha_{i}}+H_{t}^{-1} \frac{\partial^{2} H_{t}}{\partial \alpha_{i} \alpha_{j}}\right)\right\} \\
& =\sum_{t=1+\bar{k}}^{n} \frac{1}{2} \operatorname{Tr}\left\{x _ { t } x _ { t } ^ { \prime } \left[-H_{t}^{-1} \frac{\partial H_{t}}{\partial \alpha_{j}} H_{t}^{-1} \frac{\partial H_{t}}{\partial \alpha_{i}} H_{t}^{-1}\right.\right. \\
& \left.+H_{t}^{-1}\left(\frac{\partial^{2} H_{t}}{\partial \alpha_{i} \alpha_{j}} H_{t}^{-1}-\frac{\partial H_{t}}{\partial \alpha_{i}} H_{t}^{-1} \frac{\partial H_{t}}{\partial \alpha_{j}} H_{t}^{-1}\right)\right] \\
& \left.-\left(-H_{t}^{-1} \frac{\partial H_{t}}{\partial \alpha_{j}} H_{t}^{-1} \frac{\partial H_{t}}{\partial \alpha_{i}}+H_{t}^{-1} \frac{\partial^{2} H_{t}}{\partial \alpha_{i} \alpha_{j}}\right)\right\} \\
& =\sum_{t=1+\bar{k}}^{n} \frac{1}{2} \operatorname{Tr}\left\{x _ { t } x _ { t } ^ { \prime } \left[-H_{t}^{-1} \frac{\partial H_{t}}{\partial \alpha_{j}} H_{t}^{-1} \frac{\partial H_{t}}{\partial \alpha_{i}} H_{t}^{-1}\right.\right. \\
& \left.+H_{t}^{-1} \frac{\partial^{2} H_{t}}{\partial \alpha_{i} \alpha_{j}} H_{t}^{-1}-H_{t}^{-1} \frac{\partial H_{t}}{\partial \alpha_{i}} H_{t}^{-1} \frac{\partial H_{t}}{\partial \alpha_{j}} H_{t}^{-1}\right] \\
& \left.+H_{t}^{-1} \frac{\partial H_{t}}{\partial \alpha_{j}} H_{t}^{-1} \frac{\partial H_{t}}{\partial \alpha_{i}}-H_{t}^{-1} \frac{\partial^{2} H_{t}}{\partial \alpha_{i} \alpha_{j}}\right\} \text {. }
\end{aligned}
$$

\subsubsection{Consistência do estimador de ordem}

Abaixo definimos a classe de estimadores EDC para processos BEKK-GARCH considerando $N=1$ e $\theta$ como as colunas concatenadas das matrizes $\left\{A_{l}\right\}_{l=1 \cdots q}$ e $\left\{B_{l}\right\}_{l=1 \cdots p}$. Esse resultado pode ser generalizado para qualquer $N$, observando que devem ser consideradas condições para garantir que o processo seja identificável, que por Jeantheau (1998) $H_{t}$ deve ser injetiva quase certamente. De forma semelhante, é possível generalizar para matrizes $A_{l}(\theta)$ e $B_{l}(\theta)$ com condições sobre as derivadas dessas matrizes em relação a $\theta$. Esses dois casos de generalização são objetos de trabalhos futuros.

Definição 2.37. Para $K \in \mathbb{N}^{2}, r=(p, q) \in \mathbb{N}^{2}, k=\left(k_{1}, k_{2}\right) \in \mathbb{N}^{2}, \mathbb{X}$ um BEKK-GARCH(r)e $K \geq r$, definimos o estimador EDC de $r$ por

$$
\hat{r}_{e d c}=\underset{k \leq K}{\operatorname{argmin}}\left\{-\log L_{n, k}\left(\hat{\theta}_{k}\right)+\left[m^{2}\left(1+k_{1}+k_{2}\right)\right] c_{n}\right\}
$$

para $\log L_{n, k}\left(\hat{\theta}_{k}\right)$ como definida em (2.36) $e\left\{c_{n}\right\}_{n \in \mathbb{N}}$ uma sequência de números positivos. 
Os dois próximos resultados tratam da imersão dos modelos BEKK-GARCH em cadeias de Markov, que podem ser encontrados em Boussama (1998) ou Boussama, Fuchs \& Stelzer (2011).

Definição 2.38. Seja $\mathbb{X}=\left\{X_{t}\right\}_{t \in \mathbb{N}}$ um BEKK-GARCH(r) e $k \geq r$. A cadeia de Markov $k$ derivada de $\mathbb{X}$ é o processo $\mathbb{Y}=\left\{Y_{t}\right\}_{t \in \mathbb{N}}$ com valores em $\mathbb{R}^{\zeta(k)}$,

$$
Y_{t}=\left(\operatorname{vech}\left(H_{t+1}\right)^{\prime}, \operatorname{vech}\left(H_{t}\right)^{\prime}, \ldots, \operatorname{vech}\left(H_{t-k_{1}+2}\right)^{\prime}, X_{t}^{\prime}, X_{t-1}^{\prime}, \ldots, X_{t-k_{2}+1}^{\prime}\right)^{\prime}
$$

onde

$$
\zeta(k)=\frac{m(m+1)\left(k_{1}-2\right)}{2}+m\left(k_{2}-1\right)
$$

Teorema 2.39 (Boussama (1998)). Seja $\mathbb{X}=\left\{X_{t}\right\}_{t \in \mathbb{N}}$ um BEKK-GARCH(r), $k \geq r$, satisfazendo a Condição 2.7, então $\mathbb{Y}$, a cadeia de Markov $k$-derivada de $\mathbb{X}$, é Harris positiva e geometricamente ergódica.

As condições abaixo são propostas por Comte \& Lieberman (2003) e foram baseadas nas condições propostas por Jeantheau (1998) para o estabelecimento da consistência forte dos estimadores. A única exceção é o item (v), que em Comte \& Lieberman são exigidos momentos finitos de ordem 8 e nessa tese é necessário a finitude de momentos de ordem 16 para a demonstração do Lema 2.43.

Condição 2.8. Para $\mathbb{X}=\left\{X_{t}\right\}_{t \in \mathbb{N}}$ um BEKK-GARCH(r), $\theta_{r}$ seu respectivo parâmetro e $\Theta_{r}$ seu conjunto de parâmetros.

(i) $\Theta_{r}$ é compacto.

(ii) $\theta_{r}$ é ponto interior de $\Theta_{r}$.

(iii) Existe c $>0$ tal que

$$
\inf _{\theta \in \Theta_{r}} \operatorname{det}(C(\theta)) \geq c .
$$

(iv) $H_{t}(\theta)=H_{t}\left(\theta^{\prime}\right)$ q.c. se e somente se $\theta=\theta^{\prime}$.

(v) $X_{t}$ admite momentos finitos de ordem 16.

O próximo Teorema sumariza os principais resultados de Comte \& Lieberman (2003) que são utilizados a seguir. 
Teorema 2.40 (Comte \& Lieberman (2003)). Seja $\mathbb{X}=\left\{X_{t}\right\}_{t \in \mathbb{N}}$ um BEKK-GARCH(r), $k \geq r$, $\theta_{r}=\left(\alpha_{1}, \ldots, \alpha_{\gamma(k)}\right)$ seu parâmetro verdadeiro, $\hat{\theta}_{k}=\left(\hat{\alpha}_{1}, \ldots, \hat{\alpha}_{\gamma(k)}\right)$ o estimador de máxima verossimilhança de $\theta_{r}$, em que as Condições 2.7 e 2.8 são satisfeitas. Então é verdade que

(i)

$$
\lim _{n \rightarrow \infty}-\frac{D_{\theta}^{2} \log L_{n, k}\left(\theta_{r}\right)}{n}=A_{2} \quad \text { q.c. }
$$

onde

$$
A_{2}=-E\left(\frac{\partial^{2} l_{t}\left(\theta_{r}\right)}{\partial \theta \partial \theta^{\prime}}\right)
$$

(ii) $A_{2}$ é positiva definida.

(iii)

$$
\frac{D_{\theta}^{1} \log L_{n}\left(\theta_{r}, k\right)}{\sqrt{n}} \underset{D}{\rightarrow N}\left(0, A_{1}\right),
$$

onde

$$
A_{1}=E\left(\frac{\partial l_{t}\left(\theta_{r}\right)}{\partial \theta} \frac{\partial l_{t}\left(\theta_{r}\right)}{\partial \theta^{\prime}}\right)
$$

é finita e não depende de $t$.

(iv) Para todo $i, j, l \in\{1, \ldots, \gamma(k)\}$,

$$
E\left(\sup _{\left\|\theta-\theta_{r}\right\| \leq \delta}\left|\frac{\partial^{3} l_{t}(\theta)}{\partial \alpha_{i} \alpha_{j} \alpha_{l}}\right|\right)<c(\delta) .
$$

(v) Para todo $i \in\{1, \ldots, \gamma(k)\}, \frac{\partial \log L_{n, k}\left(\theta_{r}\right)}{\partial \alpha_{i}}$ é martingale com segundo momento finito.

(vi)

$$
\sqrt{n}\left(\hat{\theta}_{k}-\theta_{r}\right) \underset{D}{\rightarrow} \mathscr{N}\left(0,2 A_{2}^{-1}\right)
$$

(vii) O estimador $\hat{\theta}_{k}$ é fortemente consistente.

(viii) Existe $c_{1} \in(0, \infty)$, que não depende de t ou $\theta$, tal que

$$
\left\|H_{t}^{-1}\right\| \leq c_{1} .
$$

(ix)

$$
E\left(\left|\log \left(\operatorname{det}\left(H_{t}\left(\boldsymbol{\theta}_{r}\right)\right)\right)\right|\right)<\infty .
$$


No Teorema 2.41 e no Lema 2.42, alguns resultados de Comte \& Lieberman (2003) são ajustados para serem utilizados no que segue.

Teorema 2.41. Seja $\mathbb{X}=\left\{X_{t}\right\}_{t \in \mathbb{N}}$ um BEKK-GARCH(r), $k \geq r, \theta_{r}=\left(\alpha_{1}, \ldots, \alpha_{\gamma(k)}\right)$ seu parâmetro verdadeiro, $\hat{\theta}_{k}=\left(\hat{\alpha}_{1}, \ldots, \hat{\alpha}_{\gamma(k)}\right)$ o estimador de máxima verossimilhança de $\theta_{r}$, $\log L_{n, k}$ como definido em (2.36), $\hat{\theta}_{k} \in \Theta_{k}$ satisfazendo as Condições 2.7 e 2.8, $\dot{\theta}=s \theta_{r}+$ $(1-s) \hat{\theta}_{k}$ e $s \in[0,1]$ e $B_{\delta}\left(\theta_{r}\right) \subset \Theta_{k}$ uma vizinhança de $\theta_{r}$, então

(i) Existe $c \in(0, \infty)$, tal que, para todo $i, j, l \in\{1, \ldots, \gamma(k)\}$,

$$
\limsup _{n \rightarrow \infty}\left\|\frac{1}{n} \sum_{t=1}^{n} \sup _{\theta \in B_{\delta}\left(\theta_{r}\right)} \frac{\partial^{3} l_{t}(\theta)}{\partial \alpha_{i} \alpha_{j} \alpha_{l}}\right\| \leq c
$$

(ii)

$$
\lim _{n \rightarrow \infty}-\frac{D_{\theta}^{2} \log L_{n}(\dot{\theta})}{n}=A_{2} \quad \text { q.c. }
$$

para $A_{2}$ como definido em (2.40).

(iii)

$$
E\left(\left|\log \left[\operatorname{det}\left(H_{t}\left(\theta_{r}\right)\right)\right]+X_{t}^{\prime} H_{t}^{-1} X_{t}\right|\right)<\infty
$$

Demonstração. (i) Usando o item (iv) do Teorema 2.40 e o Teorema 2.39, basta aplicar o Teorema 2.5 .

(ii) De forma análoga à utilizada no Lema 5 de Hafner \& Preminger (2009a), como $D_{\theta}^{2} l_{t}(\theta)$ e $D_{\theta}^{3} l_{t}(\theta)$ são contínuas em relação a $\theta$ e $\hat{\theta}_{k}$ é fortemente consistente, então, pelo Teorema do valor médio

$$
\left\|\frac{1}{n} \sum_{t=1}^{n} \frac{\partial^{2} l_{t}(\dot{\theta})}{\partial \alpha_{i} \alpha_{j}}-\frac{1}{n} \sum_{t=1}^{n} \frac{\partial^{2} l_{t}\left(\theta_{r}\right)}{\partial \alpha_{i} \alpha_{j}}\right\| \leq \sup _{\theta \in B_{\delta}\left(\theta_{r}\right)}\left\{\left\|\frac{1}{n} \sum_{t=1}^{n} \frac{\partial}{\partial \theta^{\prime}}\left(\frac{\partial^{2} l_{t}(\theta)}{\partial \alpha_{i} \alpha_{j}}\right)\right\| \cdot\left\|\dot{\theta}-\theta_{r}\right\|\right\} .
$$

Usando o item (i) e a consistência forte de $\hat{\theta}$ obtemos o resultado.

(iii)

$$
\begin{aligned}
E\left(\left|\log \left[\operatorname{det}\left(H_{t}\left(\theta_{r}\right)\right)\right]+X_{t}^{\prime} H_{t}^{-1} X_{t}\right|\right) & \leq E\left(\left|\log \left[\operatorname{det}\left(H_{t}\left(\theta_{r}\right)\right)\right]\right|\right)+E\left(\left|X_{t}^{\prime} H_{t}^{-1} X_{t}\right|\right) \\
& \leq E\left(\left|\log \left[\operatorname{det}\left(H_{t}\left(\theta_{r}\right)\right)\right]\right|\right)+E\left(\left\|X_{t}\right\|^{2}\right) E\left(\left\|H_{t}^{-1}\right\|\right)
\end{aligned}
$$


que é finito usando o Teorema 2.40, itens (viii) e (ix), e a Condição 2.8.

Lema 2.42. Seja $\mathbb{X}=\left\{X_{t}\right\}_{t \in \mathbb{N}}$ um BEKK-GARCH(k) em que as Condições 2.7 e 2.8 são satisfeitas. Então existe $c_{2} \in(0, \infty)$, que não depende de $t$ ou $i$, tal que

$$
E\left(\sup _{\theta \in \Theta_{k}}\left[\left\|\frac{\partial H_{t}}{\partial \alpha_{i}}(\theta)\right\|^{8}\right]\right)<c_{2} .
$$

Demonstração. Pela demonstração do Lema A.2 de Comte \& Lieberman (2003), temos que $D_{\theta}^{1} H_{t}$ admite momentos finitos de ordem $p$ se $X_{t}$ admite momentos finitos de ordem $2 p$. Portanto, usando a Condição 2.8, temos o desejado.

Como ocorre para o caso de modelos ARCH, não foi possível satisfazer as Condições 2.1 ou 2.2 para modelos BEKK-GARCH de forma suficiente para a aplicação da Lei do Logaritmo Iterado (LLI) apresentado no Teorema 2.5. Dessa forma, foi necessário a utilização da LLI para Martingales apresentada no Teorema 2.6. A demonstração segue de forma análoga à do Lema 2.28. As diferenças significativas estão na utilização de cálculo matricial e no uso do Lema 2.42, basicamente, em substituição ao item (ii) da Condição 2.6.

Lema 2.43. Seja $\mathbb{X}=\left\{X_{t}\right\}_{t \in \mathbb{N}} u m B E K K-G A R C H(r), k \geq r, \theta_{r}=\left(\alpha_{1}, \ldots, \alpha_{\gamma(k)}\right)$ seu parâmetro verdadeiro, em que as Condições 2.7 e 2.8 são satisfeitas, então, para todo $i \in\{1, \ldots, \gamma(k)\}$,

$$
\begin{gathered}
\limsup _{n \rightarrow \infty} \frac{\frac{\partial \log L_{n, k}\left(\theta_{r}\right)}{\partial \alpha_{i}}}{\sqrt{2 n \log \log n}}=\left[E\left(\frac{\partial l_{1}\left(\theta_{r}\right)^{2}}{\partial \alpha_{i}}\right)\right]^{1 / 2} \text { q.c. } \\
\liminf _{n \rightarrow \infty} \frac{\frac{\partial \log L_{n, k}\left(\theta_{r}\right)}{\partial \alpha_{i}}}{\sqrt{2 n \log \log n}}=-\left[E\left(\frac{\partial l_{1}\left(\theta_{r}\right)^{2}}{\partial \alpha_{i}}\right)\right]^{1 / 2} \text { q.c. }
\end{gathered}
$$

$e E\left(\frac{\partial l_{1}\left(\theta_{r}\right)^{2}}{\partial \alpha_{i}}\right)$ é finito.

Demonstração. Usando o item (v) do Teorema 2.40 e assumindo $\mathscr{F}_{t-1}=\sigma\left(X_{1}, \ldots, X_{t}\right), Z_{t}=$ $t^{\delta}, \delta>1$,

$$
U_{t}=\frac{\partial l_{t}\left(\theta_{r}\right)}{\partial \alpha_{i}} \quad e \quad W_{n}=\left[n E\left(\frac{\partial l_{t}\left(\theta_{r}\right)^{2}}{\partial \alpha_{i}}\right)\right]^{1 / 2}
$$


Onde, por (2.37),

$$
\frac{\partial l_{t}\left(\theta_{r}\right)}{\partial \alpha_{i}}=\frac{1}{2} \operatorname{Tr}\left(X_{t} X_{t}^{\prime} H_{t}^{-1} \frac{\partial H_{t}}{\partial \alpha_{i}} H_{t}^{-1}-H_{t}^{-1} \frac{\partial H_{t}}{\partial \alpha_{i}}\right)
$$

Para aplicar o Teorema 2.6, basta demonstrar as hipóteses (L1-L4) que seguem.

(L1) Pela desigualdade de Chebyshev, temos que

$$
\begin{aligned}
P\left(\left|U_{t}\right|>Z_{t}\right) & =P\left(\left|\frac{\partial l_{t}\left(\theta_{r}\right)}{\partial \alpha_{i}}\right|>t^{\delta}\right) \\
& \leq \frac{1}{t^{2 \delta}} E\left(\frac{\partial l_{t}\left(\theta_{r}\right)^{2}}{\partial \alpha_{i}}\right)
\end{aligned}
$$

Usando o item (iii) do Teorema 2.40,

$$
\sum_{t=1}^{\infty} P\left(\left|U_{t}\right|>Z_{t}\right) \leq E\left(\frac{\partial l_{1}\left(\theta_{r}\right)^{2}}{\partial \alpha_{i}}\right) \sum_{t=1}^{\infty} \frac{1}{t^{2 \delta}}<\infty .
$$

Pelo Lema de Borel-Cantelli, temos que $P\left(\left\{\omega: \mathbb{I}\left(\left|U_{t}\right|>t^{\delta}\right)=1\right.\right.$ infinitas vezes $\left.\}\right)=P\left(\left\{\omega:\left|U_{t}\right|>t^{\delta}\right.\right.$ infinitas vezes $\left.\}\right)=0$

e portanto

$$
\lim _{n \rightarrow \infty} \frac{\sum_{t=1}^{n} U_{t} \mathbb{I}\left(\left|U_{t}\right|>Z_{t}\right)-E\left[U_{t} \mathbb{I}\left(\left|U_{t}\right|>Z_{t}\right) \mid \mathscr{F}_{t-1}\right]}{\sqrt{2 W_{n}^{2} \log \log W_{n}^{2}}}=0 \quad \text { q.c. }
$$

(L2)

$$
\begin{aligned}
E\left(U_{t} \mid \mathscr{F}_{t-1}\right) & =\frac{1}{2} \operatorname{Tr}\left[E\left(X_{t} X_{t}^{\prime} \mid \mathscr{F}_{t-1}\right) H_{t}^{-1} \frac{\partial H_{t}}{\partial \alpha_{i}} H_{t}^{-1}-H_{t}^{-1} \frac{\partial H_{t}}{\partial \alpha_{i}}\right] \\
& =\frac{1}{2} \operatorname{Tr}\left[H_{t} H_{t}^{-1} \frac{\partial H_{t}}{\partial \alpha_{i}} H_{t}^{-1}-H_{t}^{-1} \frac{\partial H_{t}}{\partial \alpha_{i}}\right] \\
& =0 .
\end{aligned}
$$

Além disso, pelo item (v) do Teorema 2.40,

$$
E\left(E\left(U_{t}^{2} \mid \mathscr{F}_{t-1}\right)\right)=E\left(U_{t}^{2}\right)<\infty
$$


e portanto, usando o item (i) do Teorema 2.5 (LFGN), temos que

$$
\lim _{n \rightarrow \infty} \frac{\sum_{t=1}^{n} E\left[U_{t}^{2} \mid \mathscr{F}_{t-1}\right]}{n E\left(\frac{\partial l_{t}\left(\theta_{r}\right)^{2}}{\partial \alpha_{i}}\right)}=1 \quad \text { q.c. }
$$

Usando o Teorema da Convergência Dominada, temos que

$$
\lim _{t \rightarrow \infty} E\left(U_{t} \mathbb{I}\left(\left|U_{t}\right| \leq n\right) \mid \mathscr{F}_{t-1}\right)=0 \quad \text { q.c. }
$$

Agora, considerando $\varepsilon>0$ arbitrário, é necessário encontrar uma cota superior somável em $t$ para

$$
P\left[\left|E\left(U_{t}^{2} \mid \mathscr{F}_{t-1}\right)-E\left(U_{t}^{2} I\left(\left|U_{t}\right| \leq t^{\delta}\right) \mid \mathscr{F}_{t-1}\right)\right|>\varepsilon\right]
$$

e aplicar Borel-Cantelli para obter

$$
\lim _{t \rightarrow \infty}\left[E\left(U_{t}^{2} \mathbb{I}\left(\left|U_{t}\right| \leq t^{\delta}\right) \mid \mathscr{F}_{t-1}\right)-E\left(U_{t}^{2} \mid \mathscr{F}_{t-1}\right)\right]=0 \quad \text { q.c. }
$$

e aplicar o Teorema Médio de Cesàro (Apêndice A) para concluir

$$
\begin{aligned}
\lim _{n \rightarrow \infty} \frac{\sum_{t=1}^{n} E\left[U_{t}^{2} \mathbb{I}\left(\left|U_{t}\right| \leq Z_{t}\right) \mid \mathscr{F}_{t-1}\right]-E\left[U_{t} \mathbb{I}\left(\left|U_{t}\right| \leq Z_{t}\right) \mid \mathscr{F}_{t-1}\right]^{2}}{W_{n}^{2}} & =\lim _{n \rightarrow \infty} \frac{\sum_{t=1}^{n} E\left[U_{t}^{2} \mid \mathscr{F}_{t-1}\right]}{n E\left(\frac{\partial l_{t}\left(\theta_{r}\right)}{\partial \alpha_{i}}{ }^{2}\right)} \\
& =1 \text { q.c. }
\end{aligned}
$$

Usando a desigualdade generalizada de Chebyshev

$$
\begin{aligned}
P\left[\left|E\left(U_{t}^{2} \mid \mathscr{F}_{t-1}\right)-E\left(U_{t}^{2} I\left(\left|U_{t}\right| \leq t^{\delta}\right) \mid \mathscr{F}_{t-1}\right)\right|>\varepsilon\right] & \\
\leq & \frac{1}{\varepsilon} E\left[\left|E\left(U_{t}^{2} \mid \mathscr{F}_{t-1}\right)-E\left(U_{t}^{2} I\left(\left|U_{t}\right| \leq t^{\delta}\right) \mid \mathscr{F}_{t-1}\right)\right|\right]
\end{aligned}
$$

$\mathrm{e}$

$$
\begin{aligned}
E\left[\left|E\left(U_{t}^{2} \mid \mathscr{F}_{t-1}\right)-E\left(U_{t}^{2} I\left(\left|U_{t}\right| \leq t^{\delta}\right) \mid \mathscr{F}_{t-1}\right)\right|\right] & =E\left[\left|E\left(U_{t}^{2} \mid \mathscr{F}_{t-1}\right)-E\left(U_{t}^{2} I\left(\left|U_{t}\right| \leq t^{\delta}\right) \mid \mathscr{F}_{t-1}\right) \pm E\left(U_{t}^{2} I\left(\left|U_{t}\right|>t^{\delta}\right) \mid \mathscr{F}_{t-1}\right)\right|\right] \\
& =E\left[E\left(U_{t}^{2} I\left(\left|U_{t}\right|>t^{\delta}\right) \mid \mathscr{F}_{t-1}\right)\right] \\
& =E\left[U_{t}^{2} I\left(\left|U_{t}\right|>t^{\delta}\right)\right] \\
& \leq E\left[U_{t}^{4}\right]^{1 / 2} E\left[I\left(\left|U_{t}\right|>t^{\delta}\right)\right]^{1 / 2} \\
& =E\left[U_{t}^{4}\right]^{1 / 2} \mathrm{P}\left(\left|U_{t}\right|>t^{\delta}\right)^{1 / 2}
\end{aligned}
$$


Usando (2.41), (2.45) e o Lema 2.42 para $c>0$ apropriado, obtemos

$$
E\left[\left|E\left(U_{t}^{2} \mid \mathscr{F}_{t-1}\right)-E\left(U_{t}^{2} I\left(\left|U_{t}\right| \leq t^{\delta}\right) \mid \mathscr{F}_{t-1}\right)\right|\right] \leq c \frac{1}{t^{\delta}}
$$

que é somável em $t$.

(L3) Adotando a notação $\dot{H}_{t}:=\frac{\partial H_{t}}{\partial \alpha_{i}}$, temos que

$$
\begin{aligned}
0 \leq & E\left[U_{t}^{4} \mathbb{I}\left(\left|U_{t}\right| \leq Z_{t}\right) \mid \mathscr{F}_{t-1}\right] \\
\leq & E\left[U_{t}^{4} \mid \mathscr{F}_{t-1}\right] \\
\leq & E\left\{\operatorname{Tr}\left[X_{t} X_{t}^{\prime} H_{t}^{-1} \dot{H}_{t} H_{t}^{-1}-H_{t}^{-1} \dot{H}_{t}\right]^{4} \mid \mathscr{F}_{t-1}\right\} \\
= & E\left\{\left[\operatorname{Tr}\left(X_{t} X_{t}^{\prime} H_{t}^{-1} \dot{H}_{t} H_{t}^{-1}\right)-\operatorname{Tr}\left(H_{t}^{-1} \dot{H}_{t}\right)\right]^{4} \mid \mathscr{F}_{t-1}\right\} \\
= & E\left\{\operatorname{Tr}\left(X_{t} X_{t}^{\prime} H_{t}^{-1} \dot{H}_{t} H_{t}^{-1}\right)^{4}\right. \\
& -4 \operatorname{Tr}\left(X_{t} X_{t}^{\prime} H_{t}^{-1} \dot{H}_{t} H_{t}^{-1}\right)^{3} \operatorname{Tr}\left(H_{t}^{-1} \dot{H}_{t}\right) \\
& +6 \operatorname{Tr}\left(X_{t} X_{t}^{\prime} H_{t}^{-1} \dot{H}_{t} H_{t}^{-1}\right)^{2} \operatorname{Tr}\left(H_{t}^{-1} \dot{H}_{t}\right)^{2} \\
& -4 \operatorname{Tr}\left(X_{t} X_{t}^{\prime} H_{t}^{-1} \dot{H}_{t} H_{t}^{-1}\right) \operatorname{Tr}\left(H_{t}^{-1} \dot{H}_{t}\right)^{3} \\
& \left.+\operatorname{Tr}\left(H_{t}^{-1} \dot{H}_{t}\right)^{4} \mid \mathscr{F}_{t-1}\right\}
\end{aligned}
$$

Adicionalmente,

$$
\begin{aligned}
\left|\operatorname{Tr}\left(X_{t} X_{t}^{\prime} H_{t}^{-1} \dot{H}_{t} H_{t}^{-1}\right)\right| & =\left|\operatorname{Tr}\left(H_{t}^{1 / 2} \varepsilon_{t}\left(H_{t}^{1 / 2} \varepsilon_{t}\right)^{\prime} H_{t}^{-1} \dot{H}_{t} H_{t}^{-1}\right)\right| \\
& =\left|\operatorname{Tr}\left(H_{t}^{1 / 2} \varepsilon_{t} \varepsilon_{t}^{\prime} H_{t}^{1 / 2} H_{t}^{-1} \dot{H}_{t} H_{t}^{-1}\right)\right| \\
& =\left|\operatorname{Tr}\left(\varepsilon_{t} \varepsilon_{t}^{\prime} H_{t}^{-1 / 2} \dot{H}_{t} H_{t}^{-1 / 2}\right)\right| \\
& \leq\left\|\varepsilon_{t} \varepsilon_{t}^{\prime}\right\|\left\|H_{t}^{-1 / 2}\right\|^{2}\left\|\dot{H}_{t}\right\|
\end{aligned}
$$

e, usando o Teorema 2.40 (viii) para $c \in(0, \infty)$ apropriado, temos

$$
\left|\operatorname{Tr}\left(H_{t}^{-1 / 2} \dot{H}_{t} H_{t}^{-1 / 2}\right)\right| \leq c\left\|\dot{H}_{t}\right\|
$$


Onde usamos a relação $|\operatorname{Tr}(A B C)| \leq\|A\|\|B\|\|C\|$. Portanto,

$$
\begin{aligned}
E\left[U_{t}^{4} \mathbb{I}\left(\left|U_{t}\right| \leq Z_{t}\right) \mid \mathscr{F}_{t-1}\right] \leq & E\left(\left\|\varepsilon_{t} \varepsilon_{t}^{\prime}\right\|^{4} \mid \mathscr{F}_{t-1}\right)\left\|\dot{H}_{t}\right\|^{4} \\
& +4 c E\left(\left\|\varepsilon_{t} \varepsilon_{t}^{\prime}\right\|^{3} \mid \mathscr{F}_{t-1}\right)\left\|\dot{H}_{t}\right\|^{4} \\
& +6 c^{2} E\left(\left\|\varepsilon_{t} \varepsilon_{t}^{\prime}\right\|^{2} \mid \mathscr{F}_{t-1}\right)\left\|\dot{H}_{t}\right\|^{4} \\
& +3 c^{4}\left\|\dot{H}_{t}\right\|^{4} \\
\leq & c_{1} E\left[\left\|\varepsilon_{t} \varepsilon_{t}^{\prime}\right\|^{2}+\left\|\varepsilon_{t} \varepsilon_{t}^{\prime}\right\|^{3}+\left\|\varepsilon_{t} \varepsilon_{t}^{\prime}\right\|^{4}+1 \mid \mathscr{F}_{t-1}\right]\left\|\dot{H}_{t}\right\|^{4}
\end{aligned}
$$

para $\delta_{1} \in\left(\frac{1}{2}, 1\right)$, usando as desigualdades de Chebyshev e Jensen,

$$
\begin{aligned}
\mathrm{P}\left[E\left[U_{t}^{4} \mathbb{I}\left(\left|U_{t}\right| \leq Z_{t}\right) \mid \mathscr{F}_{t-1}\right]>t^{\delta_{1}}\right] & \leq \mathrm{P}\left[E\left[\left\|\varepsilon_{t} \varepsilon_{t}^{\prime}\right\|^{2}+\left\|\varepsilon_{\varepsilon_{t}} \varepsilon_{t}^{\prime}\right\|^{3}+\left\|\varepsilon_{t} \varepsilon_{t}^{\prime}\right\|^{4}+1 \mid \mathscr{F}_{t-1}\right]\left\|\dot{H}_{t}\right\|^{4}>\frac{t^{\delta_{1}}}{c_{1}}\right] \\
& \leq \frac{c_{2}^{2}}{t^{2 \delta_{1}}} E\left\{E\left[\left(\left\|\varepsilon_{t} \varepsilon_{t}^{\prime}\right\|^{2}+\left\|\varepsilon_{t} \varepsilon_{t}^{\prime}\right\|^{3}+\left\|\varepsilon_{t} \varepsilon_{t}^{\prime}\right\|^{4}+1\right)\left\|\dot{H}_{t}\right\|^{4} \mid \mathscr{F}_{t-1}\right]^{2}\right\} \\
& \leq \frac{c_{2}^{2}}{t^{2 \delta_{1}}} E\left\{E\left[\left(\left\|\varepsilon_{t} \varepsilon_{t}^{\prime}\right\|^{2}+\left\|\varepsilon_{t} \varepsilon_{t}^{\prime}\right\|^{3}+\left\|\varepsilon_{\varepsilon_{t}} \varepsilon_{t}^{\prime}\right\|^{4}+1\right)^{2}\left\|\dot{H}_{t}\right\|^{8} \mid \mathscr{F}_{t-1}\right]\right\} \\
& \leq \frac{c_{2}^{2}}{t^{2 \delta_{1}}} E\left\{\left(\left\|\varepsilon_{\varepsilon_{t}} \varepsilon_{t}^{\prime}\right\|^{2}+\left\|\varepsilon_{t} \varepsilon_{t}^{\prime}\right\|^{3}+\left\|\varepsilon_{t} \varepsilon_{t}^{\prime}\right\|^{4}+1\right)^{2}\right\} E\left\{\left\|\dot{H}_{t}\right\|^{8}\right\} \\
& \leq \frac{c_{3}}{t^{2 \delta_{1}}} E\left[\left\|\dot{H}_{t}\right\|^{8}\right]
\end{aligned}
$$

e portanto, usando o Lema 2.42,

$$
\sum_{t=1}^{\infty} \mathrm{P}\left[E\left[U_{t}^{4} \mathbb{I}\left(\left|U_{t}\right| \leq Z_{t}\right) \mid \mathscr{F}_{t-1}\right]>t^{\delta_{1}}\right] \leq \sum_{t=1}^{\infty} \frac{c_{3}}{t^{2 \delta_{1}}} E\left[\left\|\dot{H}_{t}\right\|^{8}\right]<\infty .
$$

Usando o Lema de Borel-Cantelli,

$$
\mathrm{P}\left[E\left[U_{t}^{4} \mathbb{I}\left(\left|U_{t}\right| \leq Z_{t}\right) \mid \mathscr{F}_{t-1}\right]>t^{\delta_{1}} \quad \text { i.o. }\right]=0
$$

e logo

$$
\lim _{n \rightarrow \infty} \sum_{t=1}^{n} \frac{E\left[U_{t}^{4} \mathbb{I}\left(\left|U_{t}\right| \leq Z_{t}\right) \mid \mathscr{F}_{t-1}\right]}{W_{t}^{4}} \leq \lim _{n \rightarrow \infty} \sum_{t=1}^{n} \frac{1}{t^{2-\delta_{1}}}<\infty \quad \text { q.c. }
$$

(L4) Como $E\left(\frac{\partial l_{t}\left(\theta_{r}\right)^{2}}{\partial \alpha_{i}}\right)>0$, caso contrário $\frac{\partial l_{t}\left(\theta_{r}\right)}{\partial \alpha_{i}} \equiv 0$ q.c. e $A_{2}$ seria 0 , então, usando a 
estacionaridade, temos

$$
\begin{gathered}
\lim _{n \rightarrow \infty} \frac{W_{n}}{W_{n+1}}=\lim _{n \rightarrow \infty} \frac{\left[n E\left(\frac{\partial l_{t}\left(\theta_{r}\right)^{2}}{\partial \alpha_{i}}\right)\right]^{1 / 2}}{\left[(n+1) E\left(\frac{\partial l_{t}\left(\theta_{r}\right)^{2}}{\partial \alpha_{i}}\right)\right]^{1 / 2}}=1 \quad \text { q.c. } e \\
\lim _{n \rightarrow \infty} W_{n}=E\left(\frac{\partial l_{t}\left(\theta_{r}\right)^{2}}{\partial \alpha_{i}}\right)^{1 / 2} \lim _{n \rightarrow \infty} \sqrt{n}=\infty .
\end{gathered}
$$

Corolário 2.44. Seja $\mathbb{X}=\left\{X_{t}\right\}_{t \in \mathbb{N}} u m B E K K-G A R C H(r)$ em que as Condições 2.7 e 2.8 são satisfeitas, $k \geq r, \log L_{n}(\theta)$ como definido em (2.36), então existe $c_{5} \in(0, \infty)$ tal que

$$
\limsup _{n \rightarrow \infty} \frac{\left\|D_{\theta}^{1} \log L_{n, k}\left(\theta_{r}\right)\right\|}{\sqrt{2 \log \log n}} \leq c_{5} \quad \text { q.c. }
$$

Demonstração.

$$
\begin{aligned}
\limsup _{n \rightarrow \infty} \frac{\left\|D_{\theta}^{1} \log L_{n, k}\left(\theta_{r}\right)\right\|}{\sqrt{2 \log \log n}} & \leq \limsup _{n \rightarrow \infty} \sum_{i=0}^{\gamma(k)} \frac{\left|\frac{\partial \log L_{n, k}\left(\theta_{r}\right)}{\partial \alpha_{i}}\right|}{\sqrt{2 n \log \log n}} \\
& \leq \sum_{i=0}^{\gamma(k)} \limsup _{n \rightarrow \infty} \frac{\left|\frac{\partial \log L_{n, k}\left(\theta_{r}\right)}{\partial \alpha_{i}}\right|}{\sqrt{2 n \log \log n}} \\
& \leq \sum_{i=0}^{\gamma(k)} E\left(\frac{\partial l_{t}\left(\theta_{r}\right)^{2}}{\partial \alpha_{i}}\right)^{1 / 2} \\
& =c_{5} q . c .
\end{aligned}
$$

O Lema abaixo possui resultados técnicos que são utilizados na demonstração do Teorema 2.46, que conclui a hipótese $\mathrm{H} 1$.

Lema 2.45. Seja $\mathbb{X}=\left\{X_{t}\right\}_{t \in \mathbb{N}}$ um BEKK-GARCH(k), $i \in\{0, \ldots, \gamma(k)\}$, em que as Condições 2.7 e 2.8 são satisfeitas, então, adotando a notação $\dot{H}_{t}:=D_{\theta} H_{t}$, temos que

$$
E \sup _{\theta \in \Theta_{k}}\left[\left|\operatorname{Tr}\left(\dot{H}_{t}(\theta) H_{t}^{-1}(\theta)-X_{t} X_{t}^{\prime} H_{t}^{-1}(\theta) \dot{H}_{t}(\theta) H_{t}^{-1}(\theta)\right)\right|\right]<\infty .
$$


Demonstração. Usando o Teorema 2.40 e o Lema 2.42 temos, para uma constante $c$,

$$
\begin{aligned}
E \sup _{\theta \in \Theta_{k}}\left[\left|\operatorname{Tr}\left(\dot{H}_{t} H_{t}^{-1}-X_{t} X_{t}^{\prime} H_{t}^{-1} \dot{H}_{t} H_{t}^{-1}\right)\right|\right] & \leq E \sup _{\theta \in \Theta_{k}}\left[\left\|\dot{H}_{t}\right\|\left\|H_{t}^{-1}\right\|+\left\|X_{t} X_{t}^{\prime}\right\|\left\|H_{t}^{-1}\right\|^{2}\left\|\dot{H}_{t}\right\|\right] \\
& \leq E \sup _{\theta \in \Theta_{k}}\left[c\left\|\dot{H}_{t}\right\|+c^{2}\left\|X_{t} X_{t}^{\prime}\right\|\left\|\dot{H}_{t}\right\|\right]<\infty .
\end{aligned}
$$

Teorema 2.46. Seja $\mathbb{X}=\left\{X_{t}\right\}_{t \in \mathbb{N}}$ um BEKK-GARCH(r), $k \nsupseteq r$, $\theta_{r}$ seu parâmetro verdadeiro, $\hat{\theta}_{k}$ o estimador de máxima verossimilhança de $\theta_{r}$, em que as Condições 2.7 e 2.8 são satisfeitas, então

$$
\lim _{n \rightarrow \infty} \frac{\log L_{n}\left(\hat{\theta}_{r}\right)-\log L_{n}\left(\hat{\theta}_{k}\right)}{n}>0 \text { q.c. }
$$

Demonstração. Tomando $\bar{k} \geq k, r$, temos que

$$
\lim _{n \rightarrow \infty} \frac{\log L_{n}\left(\hat{\theta}_{r}\right)-\log L_{n}\left(\hat{\theta}_{k}\right)}{n}=\lim _{n \rightarrow \infty} \frac{\log L_{n}\left(\hat{\theta}_{r}\right)-\log L_{n}\left(\hat{\theta}_{\bar{k}}\right)+\log L_{n}\left(\hat{\theta}_{\bar{k}}\right)-\log L_{n}\left(\hat{\theta}_{k}\right)}{n} .
$$

Aplicando o Teorema 1.11, usando os resultados acima, temos que

$$
\lim _{n \rightarrow \infty} \frac{\log L_{n}\left(\hat{\theta}_{r}\right)-\log L_{n}\left(\hat{\theta}_{\bar{k}}\right)}{n}=0 \quad \text { q.c. }
$$

Observando (2.35), vemos que basta mostrar que

$$
\lim _{n \rightarrow \infty} \frac{\log L_{n}\left(\hat{\theta}_{\bar{k}}\right)-\log L_{n}\left(\hat{\theta}_{k}\right)}{n}>0 \quad \text { q.c. }
$$

considerando

$$
\log L_{n}(\theta)=\sum_{t=1+\bar{k}}^{n} l_{t}(\theta)
$$

e

$$
l_{t}(\theta)=\log (f(\theta))=\log \left(\frac{1}{\sqrt{(2 \pi)^{m / 2} \operatorname{det}\left(H_{t}\right)}} \exp \left(-\frac{1}{2} x_{t}^{\prime} H_{t}^{-1} x_{t}\right)\right)
$$

em (2.35). Assim, temos que pelo Teorema 2.41, item (iii), que

$$
E\left[\left|l_{t}\left(\theta_{r}\right)\right|\right]<\infty
$$


e portanto usando o Teorema 2.5,

$$
\lim _{n \rightarrow \infty} \frac{\log L_{n}\left(\theta_{r}\right)}{n}=\lim _{n \rightarrow \infty} \frac{\sum_{t=1+k}^{n} l_{t}\left(\theta_{r}\right)}{n}=E\left(l_{1}\left(\theta_{r}\right)\right)=c_{1}<\infty \quad \text { q.c. }
$$

Usando o Teorema do valor médio, para $\dot{\theta}=s \theta_{r}+(1-s) \hat{\theta}_{\bar{k}}, s \in(0,1), n$ suficientemente grande e $B_{\delta}\left(\theta_{r}\right)$ uma vizinhança suficientemente pequena de $\theta_{r}$, temos

$$
\begin{aligned}
\left|\frac{\sum_{t=1+\bar{k}}^{n} l_{t}\left(\hat{\theta}_{\bar{k}}\right)}{n}-\frac{\sum_{t=1+\bar{k}}^{n} l_{t}\left(\theta_{r}\right)}{n}\right| & =\left|\frac{\sum_{t=1+\bar{k}}^{n} D_{\theta}^{1} l_{t}(\dot{\theta})}{n}\left(\hat{\theta}_{\bar{k}}-\theta_{r}\right)\right| \\
& \leq \sup _{\theta \in B_{\delta}\left(\theta_{r}\right)}\left\|\frac{\sum_{t=1+\bar{k}}^{n} D_{\theta}^{1} l_{t}(\dot{\theta})}{n}\right\|\left\|\left(\hat{\theta}_{\bar{k}}-\theta_{r}\right)\right\|
\end{aligned}
$$

Aplicando o Teorema 2.5 e usando o Lema 2.45 e a consistência forte de $\hat{\theta}_{r}$,

$$
\left|\frac{\sum_{t=1+\bar{k}}^{n} l_{t}\left(\hat{\theta}_{\bar{k}}\right)}{n}-\frac{\sum_{t=1+\bar{k}}^{n} l_{t}\left(\theta_{r}\right)}{n}\right| \underset{\text { q.c. }}{\rightarrow} 0 .
$$

Por outro lado, temos que, como o estimador é de máxima verossimilhança e $\Theta_{k} \subset \Theta_{\bar{k}}$,

$$
\begin{aligned}
\lim _{n \rightarrow \infty} \frac{\log L_{n}\left(\hat{\theta}_{k}\right)}{n} & \leq \lim _{n \rightarrow \infty} \frac{\log L_{n}\left(\hat{\theta}_{\bar{k}}\right)}{n} \\
& =c_{1} \text { q.c. }
\end{aligned}
$$

e portanto,

$$
\lim _{n \rightarrow \infty} \frac{\log L_{n}\left(\hat{\theta}_{k}\right)}{n} \leq \limsup _{n \rightarrow \infty} \frac{\sum_{t=1+k}^{n} l_{t}\left(\hat{\theta}_{k}\right)}{n}=c_{2} \leq c_{1} \quad \text { q.c. }
$$

Seja $n_{i}$ uma subsequência de $n$ tal que

$$
\lim _{n \rightarrow \infty} \frac{\sum_{t=1+k}^{n_{i}} l_{t}\left(\hat{\theta}_{k}\right)}{n_{i}}=c_{2} \text { q.c. }
$$


Usando que $\Theta_{k}$ é compacto, tome $n_{j}$ uma subsequência de $n_{i}$ tal que

$$
\hat{\theta}_{k}\left(n_{j}\right) \rightarrow \bar{\theta}_{k} \in \Theta_{k} \quad \text { q.c. }
$$

Temos assim que

$$
\begin{aligned}
\lim _{n \rightarrow \infty} \frac{\log L_{n}\left(\hat{\theta}_{k}\right)}{n} & \leq \limsup _{n \rightarrow \infty} \frac{\log L_{n}\left(\hat{\theta}_{k}\right)}{n} \\
& =\lim _{n_{j} \rightarrow \infty} \frac{\log L_{n_{j}}\left(\hat{\theta}_{k}\left(n_{j}\right)\right)}{n_{j}} \text { q.c. }
\end{aligned}
$$

Aplicando o mesmo raciocínio usado em (2.46), concluímos

$$
\begin{gathered}
\lim _{n_{i} \rightarrow \infty} \frac{\log L_{n_{j}}\left(\bar{\theta}_{k}\right)}{n_{j}}=E\left(l_{1}(\bar{\theta})\right) \quad e \\
\left|\frac{\sum_{t=1+\bar{k}}^{n} l_{t}\left(\hat{\theta}_{k}\right)}{n}-\frac{\sum_{t=1+\bar{k}}^{n} l_{t}(\bar{\theta})}{n}\right| \underset{q . c .}{\rightarrow} 0 .
\end{gathered}
$$

Além disso,

$$
\lim _{n \rightarrow \infty}\left[\frac{\log L_{n}\left(\hat{\theta}_{\bar{k}}\right)}{n}-\frac{\log L_{n}\left(\hat{\theta}_{k}\right)}{n}\right] \geq E\left[\log \left(\frac{f\left(\bar{\theta}_{k}\right)}{f\left(\theta_{r}\right)}\right)\right]
$$

Por outro lado

$$
E\left[\log \left(\frac{f\left(\bar{\theta}_{k}\right)}{f\left(\theta_{r}\right)}\right)\right]
$$

é a divergência de Kullback-Leibler, que é positiva se $f\left(\bar{\theta}_{k}\right) \neq f\left(\theta_{r}\right)$, e como $\theta_{r} \notin \bar{\Theta}_{k} \subseteq \mathbb{R}^{\gamma(k)}$, temos que $\theta_{r} \neq \bar{\theta}_{k}$ e então $f\left(\bar{\theta}_{k}\right) \neq f\left(\theta_{r}\right)$. Onde concluímos

$$
\lim _{n \rightarrow \infty}\left[\frac{\log L_{n}\left(\hat{\theta}_{r}\right)}{n}-\frac{\log L_{n}\left(\hat{\theta}_{k}\right)}{n}\right] \geq E\left[\log \left(\frac{f\left(\bar{\theta}_{k}\right)}{f\left(\theta_{r}\right)}\right)\right]=c>0 .
$$

No Teorema abaixo aplicamos os resultados desenvolvidos nessa Seção para o estabelecimento da consistência forte dos estimadores de ordem EDC para modelos BEKK- 
GARCH. Como caso particular, temos a consistência forte do estimador baseado no critério de informação BIC. Nota-se que, caso seja possível encontrar cotas superiores menores para $\mathrm{H} 2$, essa classe pode ser estendida para termos de penalidade menores, da ordem de $O(\log \log n)$.

Teorema 2.47. Seja $\mathbb{X}=\left\{X_{t}\right\}_{t \in \mathbb{N}}$ um BEKK-GARCH(r) em que as Condições 2.7 e 2.8 são satisfeitas e $\hat{r}_{\text {edc }}$ como na Definição 2.37. Então $\hat{r}_{e d c}$ é fortemente consistente $\left(\hat{r}_{\text {edc }} \underset{q . c .}{\rightarrow}\right.$ ) se

$$
\lim _{n \rightarrow \infty} \frac{c_{n}}{n}=0 \quad e \quad \liminf _{n \rightarrow \infty} \frac{c_{n}}{\log \log n}=\infty
$$

Demonstração. O conjunto dos possíveis valores de $\theta_{r}, \Theta_{r}$, definido em (2.34), é aberto e $\theta_{r} \in \Theta_{r}$. De Boussama (1998), temos que o estimador de máxima verossimilhança $\hat{\theta}_{k}$ é fortemente consistente para $k \geq r$, isto é, vale (1.10). As derivadas de $\log L_{n, k}$, (2.37) e (2.38) são contínuas em relação a $\theta$ e a $x_{1}^{n}$ e portanto são mensuráveis em relação a $x_{1}^{n}$. Usando o Teoremas 2.41 e o Corolário 2.44 temos as condições satisfeitas para a aplicação do item (ii) do Teorema 1.11 e com isso temos (H2) satisfeita. A hipótese (H1) segue do Teorema 2.46. $\mathrm{O}$ resultado segue do item (i) do Teorema 1.10.

Corolário 2.48. Seja $\mathbb{X}=\left\{X_{t}\right\}_{t \in \mathbb{N}}$ um BEKK-GARCH(r) em que as Condições 2.7 e 2.8 são satisfeitas e $\hat{r}_{e d c}$ como na Definição 2.37. Então o estimador $\hat{r}_{b i c}$, como definido abaixo, é fortemente consistente.

$$
\hat{r}_{b i c}=\underset{k \leq K}{\operatorname{argmin}}\left\{-\log L_{n}\left(\hat{\theta}_{k}\right)+\frac{\left[m^{2}\left(1+k_{1}+k_{2}\right)\right]}{2} \log n\right\}
$$

para $\log L_{n}\left(\hat{\theta}_{k}\right)$ como definida em (2.35).

Demonstração. $c_{n}=\log n / 2$ e satisfaz (2.47). 


\section{Conclusão}

O método de estimação de ordem baseado no critério de informação EDC, concebido originalmente para cadeias de Markov, se mostra bastante promissor no contexto de seleção de modelos.

Além dos resultados obtidos com a definição do estimador de ordem EDC para processos AR, ARCH e BEKK-GARCH, observa-se a possibilidade de aplicação do ferramental desenvolvido para estimação de tamanho de espaço de estados e ordem de dependência ocultos em cadeias de Markov Ocultas e seleção de modelos aninhados em modelos Lineares de Espaço de Estados.

Como casos particulares, foi demonstrado nesse trabalho a consistência forte dos estimadores de ordem baseados no critério de informação BIC para os modelos ARCH e BEKKGARCH. Como ainda não existiam estudos sobre estimadores consistentes para esses casos, esses resultados se mostram altamente relevantes.

A generalização da função verossimilhança para classe de funções $\log L_{n, k}$, além de apresentar resultados práticos para a seleção de ordem utilizando o critério EDC, evidencia a possibilidade de utilização desse conceito no tratamento de outros problemas.

Para processos AR, o desenvolvimento de estimadores iniciado por Akaike (1969), com o procedimento de minimização do erro final de predição (FPE), seguido dos métodos baseados em critérios de informação (AIC, BIC e HQC), utilizavam essencialmente o mesmo radical, alterando apenas o termo de penalidade. Nessa tese, usando o ferramental desenvolvido para generalização do EDC, é proposto um novo estimador, que apresentou no geral melhor performance nas simulações numéricas realizadas.

Como trabalhos futuros, observou-se a possibilidade de existência de cotas superiores menores na hipótese $\mathrm{H} 2$, o que permitiria a extensão da classe de estimadores EDC fortemente consistentes para termos de penalidade assintoticamente menores. 


\section{Referências Bibliográficas}

Akaike, H. 1969. "Fitting autogressive models for prediction." Annals of the Institute of Statistical Mathematics 21(1):243-247.

Akaike, H. 1974. "A new look at the statistical model identification." Automatic Control, IEEE Transactions on 19(6):716-723.

Akaike, H. 1979. "A Bayesian extension of the minimum AIC procedure of autoregressive model fitting." Biometrika 66(2):237-242.

Anderson, T. W. 1994. The Statistical Analysis of Time Series. New York: Wiley.

Anderson, T. W. \& L. A. Goodman. 1957. "Statistical Inference about Markov Chains." The Annals of Mathematical Statistics 28(1):89-110.

Athreya, K. B. \& P. Ney. 1978. "A new approach to the limit theory of recurrent Markov chains." Transactions of the American Mathematical Society 245:493-501.

Athreya, K. B. \& P. Ney. 1980. "Some aspects of ergodic theory and laws of large numbers for Harris recurrent Markov chains." Colloquia Mathematica Societatis János Bolyai. Nonparametric Statistical Inference 32:41-56. Budapest, Hungary.

Baigorri, A. R., C. R. Gonçalves \& P. A. A. Resende. 2014. "Markov chain order estimation based on the chi-square divergence." The Canadian Journal of Statistics 42(4):563578 .

Bartlett, M. S. 1951. "The frequency goodness of fit test for probability chains." Proceedings of the Cambridge Philosophical Society 47(1):86-95.

Bartlett, M. S. \& D. V. Rajalakshman. 1953. "Goodness of fit tests for simultaneous autoregressive series." Journal of the Royal Statistical Society 15(1):107-124.

Basawa, I. V. \& C. C. Heyde. 1976. "Asymptotic properties of maximum likelihood estimators for stochastic processes.” The Indian Journal of Statistics 38(3):259-270.

Billingsley, P. 1961. "Statistical methods in Markov chains." The Annals of Mathematical Statistics 32(1):12-40.

Bollerslev, T. 1986. "Generalized autoregressive conditional heteroskedasticity." Journal of Econometrics 31(3):307-327. 
Bollerslev, T. 1990. "Modelling the coherence in short-run nominal exchange rates: a multivariate generalized ARCH model." Review of Economics and Statistics 72(3):498-505.

Bollerslev, T., R. F. Engle \& J. M. Wooldridge. 1988. "A capital asset pricing model with time-varying covariances." Journal of Political Economy 96(1):116-131.

Bollerslev, T., R. Y. Chou \& K. F. Kroner. 1992. "ARCH modeling in finance: A review of the theory and empirical evidence." Journal of Econometrics 52(1-2):5-59.

Boussama, F. 1998. Ergodicité, mélange et estimation dans les modèles GARCH PhD thesis Université 7 Paris.

Boussama, Farid, Florian Fuchs \& Robert Stelzer. 2011. "Stationarity and geometric ergodicity of BEKK multivariate GARCH models." Stochastic Processes and their Applications 121(10):2331 - 2360.

Carvalheira, J.G.V., R.W. Blake, E.J. Pollak, R.L. Quaas \& C.V. Duran-Castro. 1998. “Application of an Autoregressive Process to Estimate Genetic Parameters and Breeding Values for Daily Milk Yield in a Tropical Herd of Lucerna Cattle and in United States Holstein Herds." Journal of Dairy Science 81(10):2738-2751.

Choi, B. 1992. ARMA Model Identification. New York: Springer.

Cogburn, R. 1972. The Central Limit Theorem for Markov processes. In Proceedings of the 6th Berkeley Symposium on Mathematical Statistics and Probability. University of California Press pp. 485-512.

Comte, F. \& O. Lieberman. 2003. "Asymptotic theory for multivariate GARCH processes." Journal of Multivariate Analysis 84:61-84.

Csiszár, I. \& P. C. Shields. 2000. "The Consistency of the BIC Markov Order Estimator." The Annals of Statistics 28(6):1601-1619.

Doeblin, W. 1937. "Sur les propriétés asymptotiques de mouvement régis par certain types de chaînes simples.” Bull. Math. Soc. Roum. Sci. 39(1):57-115; (2), 3-61.

Doeblin, W. 1940. "Eléments d'une théorie générale des chaînes simples constantes de Markov." Annales Scientifiques de l'Ecole Normale Supérieure 57(III):61-111.

Domowitz, I. \& C. S. Hakkio. 1985. "Conditional variance and the risk premium in the foreign exchange market." Journal of International Economics 19(1-2):47-66.

Doob, J. L. 1966. Stochastic Processes. John Wiley \& Sons Inc.

Dorea, C. C. Y. 2008. "Optimal penalty term for EDC Markov chain order estimator." Annales de l'Institut de Statistique de l'Universite de Paris (l'ISUP) 52:15-26. 
Engle, R. F. 1982. "Autoregressive Conditional Heteroscedasticity with Estimates of the Variance of United Kingdom Inflation.” Econometrica 50(4):987-1007.

Engle, R. F. \& K. F. Kroner. 1995. "Multivariate simultaneous generalized ARCH.” Econometric Theory 11(1):122-150.

Engle, R. F. \& V. K. Ng. 1993. "Measuring and testing the impact of news on volatility." The Journal of Finance 48(5):1749-1778.

Feller, W. 1968. An Introduction to Probability Theory and Its Applications, Volume 1. Wiley.

Finesso, L. 1990. Consistent Estimation of the Order for Markov and Hidden Markov Chains $\mathrm{PhD}$ thesis University of Maryland.

Foster, F. G. 1953. "On the stochastic matrices associated with certain queuing processes." The Annals of Mathematical Statistics 24(3):355-360.

Francq, C. \& J. M. Zakoian. 2010. GARCH Models: Structure, Statistical Inference and Financial Applications. New York: Wiley.

Good, I. J. 1955. "The likelihood ratio test for Markoff chains.” Biometrika 42(3/4):531-533.

Hafner, C. M. \& A. Preminger. 2009a. "Asymptotic theory for a factor garch model." Econometric Theory 25(2):336-363.

Hafner, C. M. \& A. Preminger. 2009b. "On asymptotic theory for multivariate GARCH models." Journal of Multivariate Analysis 100(9):2044-2054.

Hall, P. \& C. C Heyde. 1980. Martingale Limit Theory and its Application. New York: Academic Press.

Hannan, E. J. 1980. "The estimation of the order of an ARMA process." The Annals of Statistics 8(5):1071-1081.

Hannan, E. J. \& B. G. Quinn. 1979. "The determination of the order of an autoregression." Journal of the Royal Statistical Society. Series B (Methodological) 41(2):190-195.

Harris, T. E. 1956. The existence of stationary measures for certain Markov processes. In Proceedings of the 3rd Berkeley Symposium on Mathematical Statistics and Probability. Vol. 2 University of California Press pp. 113-124.

Hoel, P. G. 1954. “A Test for Markoff Chains.” Biometrika 41(3/4):430-433.

Hughes, A. W., M. L. King \& K. T. Kwek. 2004. "Selecting the order of an ARCH model." Economics Letters 83(2):269-275.

Jeantheau, T. 1998. "Strong consistency of estimators for multivariate ARCH models." Econometric Theory null(01):70-86. 
Katz, R. W. 1981. "On some criteria for estimating the order of a Markov chain.” Technometrics 23(3):243-249.

Kolmogorov, A. N. 1936. "Anfangsgründe der Theorie der Markoffschen Ketten mit unendlichen vielen möglichen Zuständen.” Mat. Sbornik N.S. Ser pp. 607-610.

Kullback, S. 1959. Information theory and statistics. New York: John Wiley and Sons.

Logan, J. A. 1981. "A structural model of the higher-order Markov process incorporating reversion effects." Journal of Mathematical Sociology 8:75-89.

Lopes, J. S. 2005. Determinação da Ordem de uma Cadeia de Markov Usando o Critério EDC PhD thesis Universidade de Brasília, UNB, Brasil.

Maddala, G. S. \& K. Lahiri. 2009. Introduction to Econometrics. Chichester: Wiley.

Meyn, S. P. \& R. L. Tweedie. 1993. Markov Chains and Stochastic Stability. London: Springer-Verlag.

Nelson, D. B. 1991. "Conditional heteroskedasticity in asset returns: a new approach." Econometrica 59(2):349-370.

Niemi, S. \& E. Nummelin. 1982. "Central limit theorems for Markov random walks." Commentationes Physico-Mathematicae 54.

Nishii, R. 1988. "Maximum likelihood principle and model selection when the true model is unspecified." Journal of Multivariate Analysis 27(2):392-403.

Nummelin, E. 1978. “A splitting technique for Harris recurrent chains.” 43:309-318.

Nummelin, E. 1984. General Irreducible Markov Chains and Nonnegative Operators. Cambridge: Cambridge University Press.

Ogata, Y. 1980. "Maximum likelihood estimates of incorrect Markov models for time series and the derivation of AIC." Journal of Applied Probability 17(1):59-72.

Orey, S. 1959. “Recurrent Markov chains.” Pacific Journal of Mathematics 9(3):805-827.

Orey, S. 1971. Limit theorems for Markov chain transition probabilities. London: Van Nostrand Reinhold.

Ozaki, T. 1977. "On the order determination of ARIMA models." Journal of Applied Statistics 26(3):290-301.

Pegram, G. G. S. 1980. “An autoregressive model for multilag Markov chains.” Journal of Applied Probability 17:350-362.

Polansky, A. M. 2007. "Detecting change-points in Markov chains." Computational Statistics \& Data Analysis 51(12):6013-6026. 
Quenouille, M. H. 1947. "A large-sample test for the goodness of fit of autoregressive schemes." Journal of the Royal Statistical Society 110(2):123-129.

Raftery, A. E. 1985. "A model for high-order Markov chains.” J. R. Statist. Soc. B. .

Resende, P. A. A. 2009. Análise comparativa de estimadores da ordem de cadeias de Markov. Master's thesis Universidade de Brasília.

Rosenblatt, M. 1964. “Equicontinuous Markov operators.” Teor. Verojatnost. i Primenen 9:205-222.

Rosenblatt, M. 1974. "Recurrent points and transition functions acting on continuous functions." 30:173-183.

Schneider, H. 1988. Application of an autoregressive reflection model for the signal analysis of radar echoes from rotating objects. In Acoustics, Speech, and Signal Processing, 1988. ICASSP-88., 1988 International Conference on. pp. 1236-1239 vol.2.

Schwarz, G. 1978. "Estimating the Dimension of a Model." The Annals of Statistics 6(2):461-464.

Shao, J. 2007. Mathematical Statistics. New York: Springer Verlag.

Shibata, R. 1976. "Selection of the Order of an Autoregressive model by Akaike's Information Criterion." Biometrika 63:117-126.

Stelzer, R. 2008. "On the relation between the vec and bekk multivariate garch models." Econometric Theory 24(04):1131-1136.

Tong, H. 1975. "Determination of the Order of a Markov Chain by Akaike's Information Criterion." Journal of Applied Probability 12(3):488-497.

Tweedie, R. L. 1974. " $R$-theory for Markov chains on a general state space I: solidarity properties and $R$-recurrent chains." 2:840-864.

Tweedie, R. L. 1975. "Sufficient conditions for regularity, recurrence and ergodicity of Markov processes.” Math. Proc. Camb. Phil. Soc. 78:125-136.

Tweedie, R. L. 1976. “Criteria for classifying general Markov chains.” 8:737-771.

van der Vaart, A. W. 2000. Asymptotic Statistics. New York: Cambridge University Press.

Weiss, A. A. 1986. "Asymptotic theory for ARCH models: estimation and testing." Econometric Theory 2(1):107-131.

Whittle, P. 1951. Hypothesis testing in time-series analysis. Almqvist and Wiksells.

Whittle, P. 1954. Some recent contributions to the theory of stationary processes: A Study in the analysis of stationary time series. Almqvist Wiksells. 
Yule, G. U. 1921. "On the time-correlation problem, with especial reference to the variatedifference correlation method.” Journal of the Royal Statistical Society 84(4):497-537.

Zhang, F. 2011. Matrix Theory: Basic Results and Techniques. New York: Springer.

Zhao, L., C. Dorea \& C. Gonçalves. 2001. "On determination of the order of a Markov chain.” Statistical Inference for Stochastic Processes 4(3):273-282. 


\section{APÊEDICE A - Teorema Médio de Cesàro}

Teorema A.1 (Cesàro). Seja $\left\{a_{t}\right\}_{t \in \mathbb{N}}$ sequência de números reais tal que $\lim _{t \rightarrow \infty} a_{t}=$ a então

$$
\lim _{n \rightarrow \infty} \bar{a}_{n}=a
$$

$\operatorname{para} \bar{a}_{n}=\frac{1}{n} \sum_{t=1}^{n} a_{t}$

Demonstração. Dado $\varepsilon>0$, seja $j$ tal que $t>j \Rightarrow\left|a_{t}-a\right|<\varepsilon$. Então

$$
\begin{aligned}
\lim _{n \rightarrow \infty} \frac{\sum_{t=1}^{n} a_{t}}{n} & =\lim _{n \rightarrow \infty} \frac{\sum_{t=1}^{j} a_{t}}{n}+\frac{\sum_{t=j+1}^{n}\left(a_{t}+a-a\right)}{n} \\
& \leq a \lim _{n \rightarrow \infty} \frac{\sum_{t=j+1}^{n} 1}{n}+\frac{\sum_{t=j+1}^{n}\left|a_{t}-a\right|}{n} \\
& =a \lim _{n \rightarrow \infty} \frac{n-j}{n}+\varepsilon \\
& =a+\varepsilon .
\end{aligned}
$$

Usamos que

$$
\begin{aligned}
\left|\lim _{n \rightarrow \infty} \frac{\sum_{t=j+1}^{n}\left(a_{t}-a\right)}{n}\right| & \leq \lim _{n \rightarrow \infty} \frac{\sum_{t=j+1}^{n}\left|a_{t}-a\right|}{n} \\
& \leq \lim _{n \rightarrow \infty} \frac{\sum_{t=j+1}^{n} \varepsilon}{n} \\
& \leq \varepsilon \lim _{n \rightarrow \infty} \frac{n-j}{n} \\
& =\varepsilon .
\end{aligned}
$$


Analogamente, demonstra-se que $\lim _{n \rightarrow \infty} \frac{\sum_{t=1}^{n} a_{t}}{n} \geq a-\varepsilon$.

Proposição A.2. Seja $\left\{a_{t}\right\}_{t \in \mathbb{N}} e\left\{b_{t}\right\}_{t \in \mathbb{N}}$ sequências de números reais tais que $\lim _{t \rightarrow \infty}\left|b_{t}-a_{t}\right|=$ $0 e\left(\sum_{1}^{n} a_{t}\right) / n \rightarrow a$, então

$$
\lim _{n \rightarrow \infty} \frac{\sum_{t=1}^{n} b_{t}}{n}=a .
$$

Demonstração. Como $\lim _{t \rightarrow \infty}\left|a_{t}-b_{t}\right|=0$, usando o Teorema A.1, temos que

$$
\lim _{n \rightarrow \infty} \frac{\sum_{t=1}^{n}\left|a_{t}-b_{t}\right|}{n}=0
$$

então

$$
\begin{aligned}
\lim _{n \rightarrow \infty} \frac{\sum_{t=1}^{n} b_{t}}{n} & =\lim _{n \rightarrow \infty} \frac{\sum_{t=1}^{n} a_{t}+b_{t}-a_{t}}{n} \\
& =\lim _{n \rightarrow \infty} \frac{\sum_{t=1}^{n} a_{t}}{n}+\frac{\sum_{t=1}^{n}\left(a_{t}-b_{t}\right)}{n} \\
& =a .
\end{aligned}
$$




\section{APÊNDICE B - Desigualdade Generalizada de Chebyshev}

Teorema B.1 (Desigualdade generalizada de Chebyshev). Se $\varepsilon>0$ e $r>0$, então

$$
\mathrm{P}(|X|>\varepsilon) \leq \frac{E\left(|X|^{r}\right)}{\varepsilon^{r}} .
$$

Demonstração.

$$
\begin{aligned}
E\left(|X|^{r}\right) & =\int|X|^{r} d P \\
& \geq \int_{|x|>\varepsilon}|X|^{r} d P \\
& \geq \int_{|x|>\varepsilon} \varepsilon^{r} d P \\
& =\varepsilon^{r} P(|X|>\varepsilon)
\end{aligned}
$$

Então,

$$
P(|X|>\varepsilon) \leq \frac{E\left(|X|^{r}\right)}{\varepsilon^{r}} .
$$

